\title{
RAPIDLY LOCATING AND ACCURATELY TRACKING THE CENTER OF MASS USING STATICALLY EQUIVALENT SERIAL CHAINS
}

\author{
Dissertation \\ Submitted to \\ The School of Engineering of the \\ UNIVERSITY OF DAYTON \\ In Partial Fulfillment of the Requirements for \\ The Degree of \\ Doctor of Philosophy in Engineering
}

By

Ali Soud Almandeel

UNIVERSITY OF DAYTON

Dayton, Ohio

December, 2015 


\section{RAPIDLY LOCATING AND ACCURATELY TRACKING}

\section{THE CENTER OF MASS USING STATICALLY EQUIVALENT SERIAL CHAINS}

Name: Almandeel, Ali Soud

\section{APPROVED BY:}

Andrew P. Murray, Ph.D.

Advisor Committee Chairman

Professor, Department of Mechanical

and Aerospace Engineering
David H. Myszka, Ph.D.

Committee Member

Associate Professor, Department of

Mechanical and Aerospace Engineering
Raúl Ordóñez, Ph.D.

Committee Member

Professor, Department of Electrical and

Computer Engineering
David A. Perkins, Ph.D.

Committee Member

Lecturer, Department of Mechanical and

Aerospace Engineering
John G. Weber, Ph.D.

Associate Dean

School of Engineering
Eddy M. Rojas, Ph.D., M.A., P.E.

Dean, School of Engineering 
(C) Copyright by

Ali Soud Almandeel

All rights reserved

2015 


\begin{abstract}
RAPIDLY LOCATING AND ACCURATELY TRACKING

THE CENTER OF MASS USING STATICALLY EQUIVALENT SERIAL CHAINS
\end{abstract}

Name: Almandeel, Ali Soud

University of Dayton

Advisor: Dr. Andrew P. Murray

This dissertation presents a center of mass $(\mathrm{CoM})$ estimation technique that uses the statically equivalent serial chain (SESC). A SESC is a representation of any multilink branched chain whose end-effector locates the CoM. Identifying the center of mass location provides a significant aid in controlling the balance of humanoid robots. Additionally, in humans this location is an essential parameter in postural control and is critical in assessing rehabilitation. Anthropometric tables have been complied for this identification but their accuracy is readily questioned. The method starts with an experimental phase involving a force plate and a motion capture system (MoCap) to construct a model to predict the CoM location. Subsequent motion of the subject updates the CoM model based on MoCap information without need of a force plate, overcoming disadvantages of some other CoM estimation methods. The node-based SESC model is developed to best integrate with Kinect and the likely MoCap systems that will be developed in the near future.

The results show that the SESC methodology allows rapid and accurate real time estimation of the CoM. The transfer-ability of the SESC parameters among subjects with similar body structure using the donor model is also presented. The donor model allow subjects to perform fewer postures 
in the experimental phase to generate a SESC. The donor model facilitates the identification of a SESC for subjects with limited mobility.

This work includes the CoM estimation for human subjects using low-end and high-end MoCap and force plate sensors. The high-end and low-end MoCap and force plate sensors are used for cross validation and to show that the method is applicable to both systems. Additionally, the presence of a static body in the workspace (a walker or chair, for example) to create stability in test subjects is presented. The introduction of the static body aids in balance and stability and adds more postures to agile subjects. Furthermore, the effect to the SESC parameters by the modification of the node-based SESC technique to allow an arbitrary location of the CoM associated with the torso is presented. The addition of a moving frame to any specific node accounts for CoMs that do not lie along the line connecting successive nodes. Finally, the accuracy of the vertical component of the CoM is also considered. 
To my parents, Soud and Monerah RIP, my wife, May, and my brothers and sisters 


\section{ACKNOWLEDGMENTS}

Pursuing my PhD at the University of Dayton was an interesting journey, one that is filled with enormous amount of experience starting from a graduate student and evolving into a $\mathrm{PhD}$ candidate. Accomplishing my dissertation would not have been possible without the countless blessings of The Almighty God and the support of many people. I would like to thank my family, friends, advisers, and committee members for their countless support during my studies.

In the beginning, I would to thank Professor Andrew Murray and Dr. David Myszka for serving as my advisors during my graduate studies. Their knowledge along with their enormous effort and support was the key in producing this dissertation. It was a pleasure to work under their supervision and I am grateful for knowing them.

I would like to thank my other committee members, Professor Raúl Ordóñez and Dr. David Perkins for being my committee members and for their insights and input to my dissertation. Their insights helped me to improve my dissertation and I am grateful for knowing them.

The enormous support of my family was one of the important keys to success. I would like to thank all of my brothers and sisters for being patient during my education journey. A huge thank for my wife, May, for being patient through the journey and for her continuous effort and support.

I would like to thank Kuwait and the Public Authority for Applied Education and Training for funding my education and research. 
Lastly, I would like to thank all my fellow DIMLabers and a special thank for Saleh Almestiri, Bingjue Li, Hussein Ali, Alex Hazlett, Suresh Kanathala, Vijay Krishna, Anna Swigert, and Ethan Vantilburg. 


\section{TABLE OF CONTENTS}

ABSTRACT $\ldots \ldots \ldots \ldots \ldots \ldots \ldots \ldots \ldots \ldots \ldots \ldots \ldots \ldots \ldots$ iii

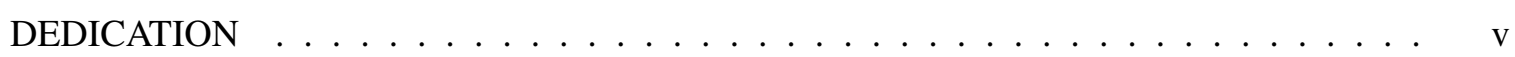

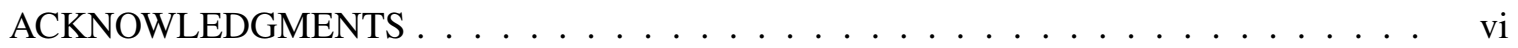

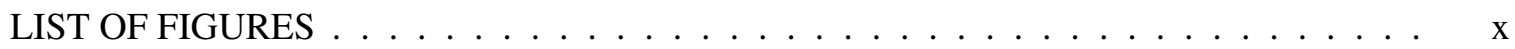

LIST OF TABLES $\ldots \ldots \ldots \ldots \ldots \ldots \ldots \ldots \ldots \ldots \ldots \ldots \ldots \ldots \ldots$

I. INTRODUCTION $\ldots \ldots \ldots \ldots \ldots \ldots \ldots \ldots \ldots \ldots \ldots \ldots \ldots$

1.1 Methods for Estimating the Center of Mass $\ldots \ldots \ldots \ldots \ldots$

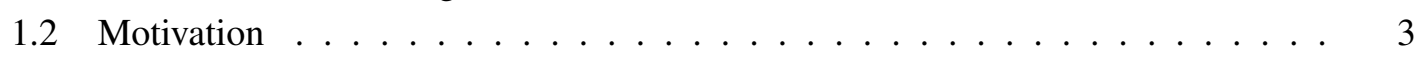

1.3 The Statically Equivalent Serial Chain Method $\ldots \ldots \ldots \ldots \ldots \ldots$

1.3.1 SESC Modeling for Planar Case . . . . . . . . . . . . . 5

1.3.2 Frame-Based SESC Modeling for Spatial Case . . . . . . . . . 6

1.4 Movement Analysis Systems . . . . . . . . . . . . . . . . . . . 10

1.4.1 Marker-Based Motion Capture Systems . . . . . . . . . . . . . 10

1.4.2 Markerless Motion Capture Systems _ . . . . . . . . . . . . 11

1.4 .3 Force Platforms . . . . . . . . . . . . . . . . . . 13

1.4.4 The Wii Balance Board . . . . . . . . . . . . . . . . 14

1.5 Center of Pressure and Center of Mass . . . . . . . . . . . . . . . . 14

1.5 .1 Human Balance . . . . . . . . . . . . . . . 16

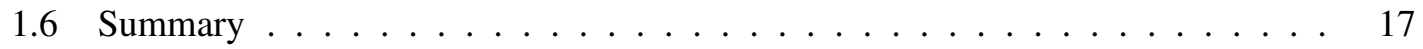

II. FORCE PLATE AND MOTION CAPTURE PARAMETER IDENTIFICATION . . 19

2.1 Experiment Protocol . . . . . . . . . . . . . . . . . . . . . . 19

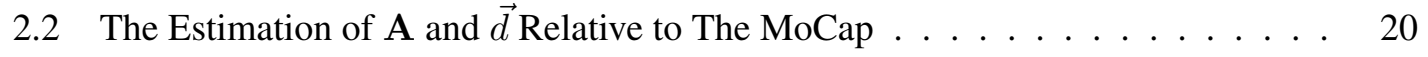

III. NODE-BASED STATICALLY EQUIVALENT SERIAL CHAIN . . . . . . . . . . . 24

3.1 Human Skeleton Model $\ldots \ldots \ldots$ 
3.2 Model Construction of a Human Subject where A and $\vec{d}$ are Known . . . . . . . 24

3.3 Using a Donor Model to Generate the SESC of a New Subject . . . . . . . . . . 30

3.4 Constructing a SESC in the Presence of a Static Body . . . . . . . . . . . . . . . . 32

3.5 Arbitrary Location of the CoM Associated with the Torso . . . . . . . . . . . . 34

3.6 Model Construction of a Human Subject where A and $\vec{d}$ are Unknown . . . . . . 37

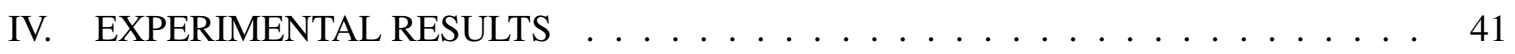

4.1 Experimental Protocol . . . . . . . . . . . . . . . . . . 41

4.2 Method Validation Where $\mathbf{A}$ and $\vec{d}$ are Known $\ldots \ldots \ldots \ldots$

4.2.1 Method Validation using Kinect/WBB Sensors . . . . . . . . . . . . . . 44

4.3 Method Validation of The Donor Model using Kinect/WBB Sensors . . . . . . . 56

4.4 Method Validation Where $\mathbf{A}$ and $\vec{d}$ are Unknown . . . . . . . . . . . . . . 64

4.4.1 Method Validation using Kinect/WBB Sensors . . . . . . . . . . . . . . 64

4.4.2 Method Validation using VICON/AMTI-OR6 Sensors With a Static Body

In the Work-space . . . . . . . . . . . . . . . . . 67

4.5 Accuracy of the Vertical Component of the CoM . . . . . . . . . . . 70

V. CONCLUSIONS AND FUTURE WORK . . . . . . . . . . . . . . . 75

5.1 Contributions of the Dissertation . . . . . . . . . . . . . 75

5.2 Future Work . . . . . . . . . . . . . . . . 76

\section{APPENDICES:}

A. INSTRUCTIONAL GUIDE FOR THE EXPERIMENTAL SETUP AND POSCAP, THE POSTURE CAPTURE SOFTWARE . . . . . . . . . . . . . . 78

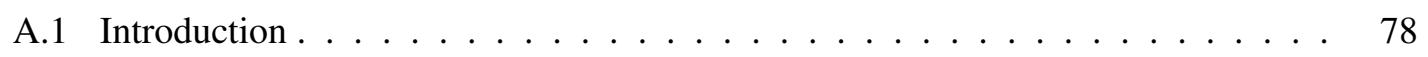

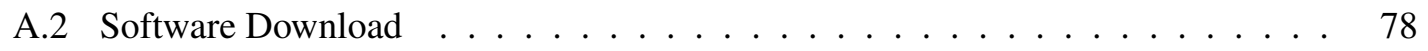

A.3 Connecting the WBB to the $\mathrm{PC} \ldots \ldots \ldots \ldots \ldots$

A.4 Kinect and WBB Placement . . . . . . . . . . . . . . . . . . 80

A.5 Parameter Identification using PosCap . . . . . . . . . . . . . . . . 81

A.6 Capturing Postures using the PosCap . . . . . . . . . . . . . . . 82

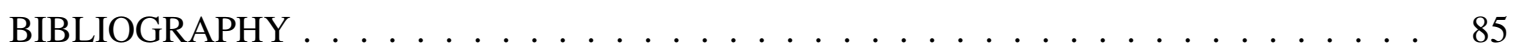




\section{LIST OF FIGURES}

1.1 (a) The kinematic and static parameters of a planar serial chain system. (b) The SESC model of the planar serial chain system. . . . . . . . . . . . . . . . . . 6

1.2 An articulated branched spatial system composed of 4 moving rigid bodies. . . . .

1.3 The kinematic and static parameters of the spatial branched chain system shown in

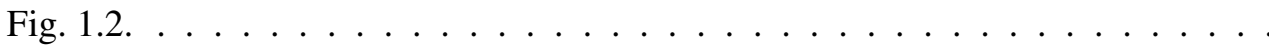

1.4 The SESC model of the spatial branched chain system shows its terminal point locating the full system's CoM. . . . . . . . . . . . . . .

1.5 (a) Kinect sensor components including the IR projector, the RGB camera, and the IR camera [1]. (b) WBB sensor components showing the 4 pressure sensors (strain gauges) at each corner $[2] \ldots \ldots \ldots \ldots \ldots$

1.6 (a) The VICON passive marker is made from a spherical retro-reflective material capable of reflecting the light emitted from the camera back to the camera lens [3]. (b) The Optotrak active marker can emit its own light pattern at a given frequency and does not depend on illumination $[4] \ldots \ldots \ldots \ldots$

1.7 (a) A markerless based motion capture system does not require the use of markers for motion detection and tracking. (b) A marker-based motion capture system relies on the markers for motion detection and tracking. . . . . . . . . . . . . . .

1.8 (a) Kistler-9281E, a piezoelectric force plate [5] and (b) AMTI-OR6-7, a strain gauge force plate [6].

1.9 A model of a human as an inverted pendulum which includes: the $\operatorname{CoM}(\vec{C})$, the COP position (b), the CoM projection position $(a)$, the body weight $(W)$, the tangent vector of the inertial force $\left(F^{i T}\right)$, the normal vector of the inertial force $\left(F^{i N}\right)$, the inertial torque $\left(T^{i}\right)$, the height of CoM $(c)$, the height of the inverted pendulum $(d)$, and the ground reaction force $(R) \ldots \ldots \ldots \ldots$ 
2.1 The actual orientation of the Kinect and the WBB reference frames. The Kinect's reference frame has the $z$ axis pointing toward the user and the WBB's reference frame has the $z$ axis pointing downward matching the direction of gravity. . . . . .

2.2 The experimental setup where the target weight is placed on the top of the WBB facing the Kinect. The kinematic and static parameters of the target weight are shown. 21

2.3 The parameter identification experiment to determine $\mathbf{A}$ and $\vec{d}$ includes the WBB, the Kinect and the target weight. . . . . . . . . . . . . . . .

2.4 Top view of the target weight as seen on the WBB, during the parameter identification experiment, in 9 different positions to record its location via the Kinect and WBB.

3.1 (a) The skeletal model generated by the Kinect sensor is composed of 19 links. Note that the links and nodes in blue are neglected. (b) The reduced human skeleton model is composed of 13 links. . . . . . . . . . . . . . . . . .

3.2 The skeleton model of a human subject composed of 13 rigid links. The kinematic and static parameters of the subject are shown. . . . . . . . . . . . . .

3.3 The node-based SESC relying solely on the MoCap system to locate the CoM of the human subject is composed of 13 rigid links. . . . . . . . . . .

3.4 The skeleton model of a human subject composed of 13 links including the static body to provide the needed balance/stability for subjects or while preforming unconventional body postures. . . . . . . . . . . . . . . .

3.5 The skeleton model of a human subject composed of 13 links. The kinematic and static parameters of the subject are show as well as the moving frame attached to

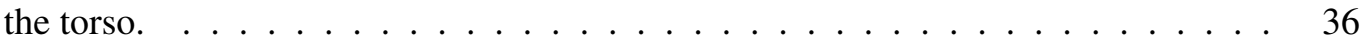

4.1 The flow chart detailing the process of determining the near static postures. . . . .

4.2 The SESC model of Subject 1 where the SESC, depicted by the green dashed lines,

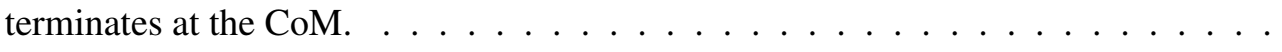

4.3 The average distance of the CoM for Subject 1 for the testing set of 30 postures as a function of the number of experimental postures. . . . . . . . . .

4.4 The 13 distinct postures performed by Subject 2 and Subject 3 during the SESC

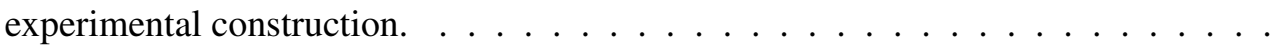


4.5 The SESC model of Subject 2 where the SESC, depicted by the green dashed lines, terminates at the CoM. . . . . . . . . . . . . . . . 51

4.6 The average distance of the CoM for Subject 2 for the testing set of 30 postures as a function of the number of experimental postures. . . . . . . . . . . . . . . 54

4.7 The SESC model of Subject $3 \ldots \ldots \ldots \ldots$. . . . . . . . . . . . . . 55

4.8 The average distance of the CoM for Subject 3 as a function of the number of experimental postures using the two different training and testing sets. . . . . . . 58

4.9 The SESC model of subject $4 \ldots \ldots \ldots \ldots \ldots$

4.10 The nine limited motion postures of Subject 4 during the SESC experimental construction. $\ldots . \cdots$

4.11 The SESC model of Subject $5 . \ldots \ldots \ldots \ldots \ldots$

4.12 The average distance of the CoM for Subject 5 for the testing set of ten postures as

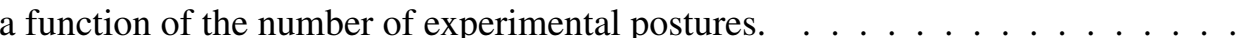

4.13 The VICON/AMTI-OR6 setup during the experimental phase shows the static body (red object not currently on the force plates used to assist in producing various number of postures while being fixed in one location on top of the force plate surface) and placement of the 35 passive markers following the Plug-in-Gait (PiG) template to define the body segments. . . . . . . . . . . . . . .

4.14 The average distance of the CoM for Subject 6 for the testing set of twenty two postures as a function of the number of experimental postures. . . . . . . . .

4.15 The two postures with more horizontal components used to validate the vertical component of the CoM of Subject 1, (a) posture number 56 and (b) posture number

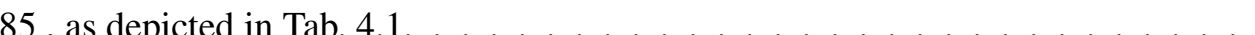

4.16 The two postures with more horizontal components used to validate the vertical component of the CoM of Subject 2, (a) posture number 86 and (b) posture number 92, as depicted in Tab. 4.4. . . . . . . . . . . . . . . . . . . . . .

4.17 The two postures with more horizontal components used to validate the vertical component of the CoM of Subject 3, (a) posture number 7 and (b) posture number 31, as depicted in Tab. $4.5 \ldots \ldots \ldots \ldots \ldots$ 
4.18 The two postures with more horizontal components used to validate the vertical component of the CoM of Subject 4, (a) posture number 12 and (b) posture number 27, as depicted in Tab. 4.6. . . . . . . . . . . . . . . . . . .

4.19 The two postures with horizontal-like components used to validate the vertical component of the CoM of Subject 6 in experiment F, (a) posture number 17 and (b) posture number 18 , as depicted in Tab. $4.12 \ldots \ldots \ldots \ldots$. . . . . . . . . . . . . . .

A.1 Click on the code or scan to open the link for downloading the Kinect SDK. . . . 79

A.2 Click on the code or scan to open the link for downloading the Visual Studio software. 79

A.3 The schematic of the WBB sensor, (a) the synchronizing button used to connect the device to the $\mathrm{PC}$ is located at the middle right, (b) the sync button is located adjacent to the battery compartment. . . . . . . . . . . . . . . . . . . 80

A.4 The placement of Kinect and WBB sensors. . . . . . . . . . . . . . . . . . 81

A.5 The Posture Capture software (PosCap), best used when the subject is wearing a tight, non-reflective outfit and required to be standing on the WBB. . . . . . . . . . 


\section{LIST OF TABLES}

4.1 The CoM positions for Subject 1 as predicted by the node-based SESC method for the 94 postures using the data from the Kinect/WBB sensors. The table includes the deviations of the predicted CoM from the measured CoM for postures 1 through 47 .

4.2 The CoM positions continued for Subject 1 for postures 48 through 94. . . . . . . .

4.3 The CoM positions for Subject 2 as predicted by the node-based SESC method for the 94 postures using the data from the Kinect/WBB sensors. The table includes the deviations of the predicted CoM from the measured CoM for postures 1 through 47 .

4.4 The CoM positions continued for Subject 2 for postures 48 through 94. . . . . . . .

4.5 The CoM positions for Subject 3 as predicted by the node-based SESC method for the 32 postures using the data from the Kinect/WBB sensors. The table includes the deviations of the predicted CoM from the measured CoM. . . . . . . . . . .

4.6 The CoM positions for Subject 4 as predicted by the node-based SESC method for the 30 postures using the data from the Kinect/WBB sensors. The table includes the deviations of the predicted CoM from the measured CoM. . . . . . . . .

4.7 The CoM positions for Subject 4 as predicted by the node-based SESC method for the four limited motion postures using the data from the Kinect/WBB sensors. The table includes the deviations of the predicted CoM from the measured CoM. . . . . .

4.8 The CoM positions for Subject 4 as predicted by the node-based SESC donor model, from Donor 1, for the four limited motion postures using the data from the Kinect/WBB sensors. The table includes the deviations of the predicted CoM from the measured

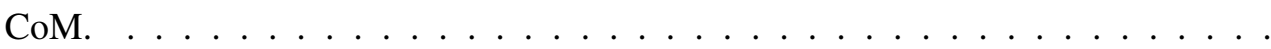


4.9 The CoM positions for Subject 4 as predicted by the node-based SESC donor model, from Donor 2, for the four limited motion postures using the data from the Kinect/WBB sensors. The table includes the deviations of the predicted CoM from the measured

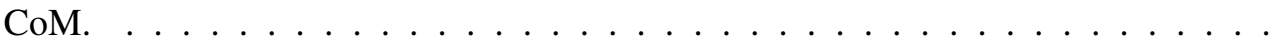

4.10 The average error and the RMSE of the CoM positions for Subject 4 as predicted by the node-based SESC versus the one predicted by the donor model, from Donor 1 and Donor 2, for the four limited motion postures using the data from the Kinect/WBB sensors. . . . . . . . . . . . . . . . . . . . . 64

4.11 The CoM positions for Subject 5 as predicted by the node-based SESC method for the ten postures using the data from the Kinect/WBB sensors. The table includes the deviations of the predicted CoM from the measured CoM. . . . . . . . . . . . .

4.12 The CoM positions for Subject 6 as predicted by the node-based SESC method for the twenty two postures using the data from Vicon/AMTI-OR6 sensors. The table includes the deviations of the predicted CoM from the measured CoM. . . . . . . . . 


\section{CHAPTER I}

\section{INTRODUCTION}

In humanoid systems, estimating the center of mass (CoM) provides an important aid in the control of static balance and posture [7]. For human beings, the stability of human motion during standing and walking is highly influenced by the position of the individual's CoM and center of pressure $(\mathrm{CoP})$. The CoM for humans is an indicator of stability and is an important parameter in the postural control system that relies on the information received from the visual, vestibular, and somatosensory systems to maintain balance [8]. Winter [9] showed that the average CoP is equal to the average CoM projection when standing as still as possible. Furthermore, computing the CoM can prove critical in assessing rehabilitation success [10], in pathology detection [11], in describing gaits [12], in stroke rehabilitation [13] and in elite sports training [14]. Estimating the CoM position is a complex issue, for example, the process may involve the estimation of limb weight and composition or involve the treatment of motion data by optimization-based integration approaches $[15,16,17]$.

Human body segment parameters can be obtained from anthropometric tables [18], but are limited to subjects similar to the sample population. Therefore, their accuracy is readily questioned when estimating an individual's CoM due to differences in subject age, race, and physical fitness 
level [15], [17]. Medical imaging techniques such as magnetic resonance imaging (MRI) or computer tomography (CT) [15] can be used to improve the table's estimates. The use of these techniques increases the cost and complexity of the method.

\subsection{Methods for Estimating the Center of Mass}

The CoM of a distribution of masses is the unique point where the mass-weighted relative position vectors sum to zero. The CoM for an articulated system of rigid bodies can be expressed as the weighted sum of the individual body's masses. Observe that for a static system, using the single point identified by the CoM of each individual rigid body produces perfectly accurate models.

The literature contains several methods for predicting the CoM position of an articulated system of bodies. One of the most common methodologies is to estimate the horizontal location of the CoM by recording the CoP, using data generated via a force platform, and then using this information in Newton's equations [19, 20]. Venture et al. [21] proposed an identification method capable of providing specific subject mass and inertial parameters of each body segment that requires a highend motion capture system and force platform due to the need for accurate measurement of segment acceleration. Schepers et al. [16] combined the CoP trajectory with the double integrated CoM acceleration to estimate the CoM continuously while walking. This method requires all movements to be performed while wearing instrumented shoes to provide continuous recording of the CoP position. As such, the use of these methods is not suitable for in-home rehabilitation due to high equipment costs. Betker et al. developed two methods to estimate the CoM using a hybird genetic algorithm sum of sines model [22] and using a feedforward backpropagation neural network model [23]. Although these two methods produce acceptable CoM estimation error, they remain sensitive to the complexity of the task or motion. 
Real-time estimation of the CoM requires tracking of the subject's movements. This is typically obtained with expensive motion capture equipment and/or a force platform. This type of environment limits the use of the CoM estimation by not being widely applicable due to its long set up process (the placement of whole body markers) and cost. Furthermore, real-time tracking and display of the CoM can be desired as visual feedback, for example, in balance training [24], in elite sports training [14], and in decreasing the walking energy cost after stroke rehabilitation [25].

\subsection{Motivation}

The use of the statically equivalent serial chain (SESC) approach $[26,27,28]$ for estimating the CoM of articulated rigid body systems can overcome the difficulties of the previous methods. Unlike the previous approaches which seek an estimation of the CoM location based on continuous data recording from force-plates, the SESC method consists of experimentally constructing the model and then using it as a predictor for the CoM. The construction of the model requires an initial set of experiments after which the CoM may be updated based solely on current joint angles or nodes in the system, neither of which requires a force plate. SESC is based on a manipulation of the equation for determining the CoM [29, 30]. Espiau and Boulic [29] described the CoM of any branched architecture with a resemblance to the forward kinematics of a serial chain.

A similar concept is noted in the method of principal vectors introduced by O. Fischer [30]. By this method, the CoM is given by the sum of a series of vectors directed along a mechanism's links. The sum of these principle vectors results in an augmented mechanism containing a point which terminates at the system's CoM. Fischer and Artobolevskii [31] introduced different versions of this construction, and the method is described in the literature by many authors [32, 33, 34, 35]. Another similar method was introduced by Berkof and Lowen [36], the method of linearly independent vectors. They applied this method to four-bar and six-bar linkages and were able to show that the 
masses of these links can be redistributed in such a way causing the total CoM to become stationary. Similarly, the SESC method creates a virtual chain where the CoM of the entire system is expressed as the terminal point of a serial chain.

A substantial advantage of CoM estimation using the SESC is that it does not depend on the knowledge of the system's static parameters including the total height, the masses of individual bodies or links, or the relative CoM location of individual bodies or links. Furthermore, experimental determination of a SESC does not require knowledge of any lengths in the system. The SESC's experimental construction only depends on the total mass, and the system's kinematic architecture which includes the joint types and the order in which they are connected. The generated SESC has the same number of degrees of freedom as the original system and its joint movements correspond to those of the original system. This modeling methodology has been applied to many examples and has been generalized to address the potential challenges faced in constructing the SESC for an adequately complex chain of bodies like, for example, in humanoid robots [27] and in human beings [28]. The SESC method is studied herein due to the simplicity of its calculation and subject specific nature.

The experimental construction of the SESC may utilize any MoCap system that is capable of motion tracking and any force plate for CoP measurements. To validate the presented methodology, the Kinect and the WBB are primarily used due to their portability, accessibility and simplicity, noting that the proposed methodology does not depend specifically on the Kinect/WBB sensors. The Kinect is used for markerless tracking, reconstructing the subject in $3 \mathrm{D}$, and providing the joint positions for the kinematic analysis. The WBB is used for CoP measurement during the SESC calibration phase. The Microsoft Kinect and the Nintendo WBB have been utilized as an affordable alternative for estimating the $\operatorname{CoM}[37,38,39]$. Accordingly, encouraging results emerged from being tested, respectively, in measurement of gait characteristics and in rehabilitation of patients 
with balance problems. The VICON MoCap system and AMTI-OR6 force plate are used for cross validation of the methodology. Relevant details about the Kinect and the WBB sensors as compared to laboratory-grade sensors are covered in Subsection 1.4.

\subsection{The Statically Equivalent Serial Chain Method}

The CoM of an articulated system of rigid bodies can be expressed as the terminal point of a virtual serial chain. Such a chain is termed the statically equivalent serial chain (SESC). A simple planar example is used to motivate the concept. The generalization to the spatial case, including branched chains, is then presented.

\subsubsection{SESC Modeling for Planar Case}

The position of the terminal point for the system of two bodies shown in Fig. 1.1a is

$$
\vec{E}=l_{1}\left\{\begin{array}{c}
\cos \theta_{1} \\
\sin \theta_{1}
\end{array}\right\}+l_{2}\left\{\begin{array}{c}
\cos \theta_{2} \\
\sin \theta_{2}
\end{array}\right\} \text {. }
$$

The CoM of the system is

$$
\vec{C}=\frac{m_{1}}{M}\left(c_{1}\left\{\begin{array}{c}
\cos \theta_{1} \\
\sin \theta_{1}
\end{array}\right\}\right)+\frac{m_{2}}{M}\left(l_{1}\left\{\begin{array}{c}
\cos \theta_{1} \\
\sin \theta_{1}
\end{array}\right\}+c_{2}\left\{\begin{array}{c}
\cos \theta_{2} \\
\sin \theta_{2}
\end{array}\right\}\right)
$$

where, $m_{i}$ is the mass of link $i, c_{i}$ is the location of the CoM of link $i$, and $M$ is the total mass of the system. The CoM of link $i$ is assumed to lie on the line segment shown in Fig. 1.1. Rewriting (1.2),

$$
\vec{C}=l_{1}^{*}\left\{\begin{array}{c}
\cos \theta_{1} \\
\sin \theta_{1}
\end{array}\right\}+l_{2}^{*}\left\{\begin{array}{c}
\cos \theta_{2} \\
\sin \theta_{2}
\end{array}\right\}
$$

where,

$$
l_{1}^{*}=\frac{m_{1} c_{1}+m_{2} l_{1}}{M}, \quad l_{2}^{*}=\frac{m_{2} c_{2}}{M} .
$$

Observe the $l_{i}^{*}$ in (1.4) are functions of only the link properties. Thus, the CoM location of the chain is modeled by the terminal point of a virtual planar serial chain of appropriate size as depicted by 
the blue dashed line in Fig. 1.1b. The terminal point of this chain is the system's CoM. There is a readily noted similarity between (1.1) and (1.3). From Fig. 1.1b note that the SESC maintains the same number of degrees of freedom as the original articulated serial chain. Further, observe that the links of the serial chain created by the SESC correspond to the same orientation as the original serial chain.

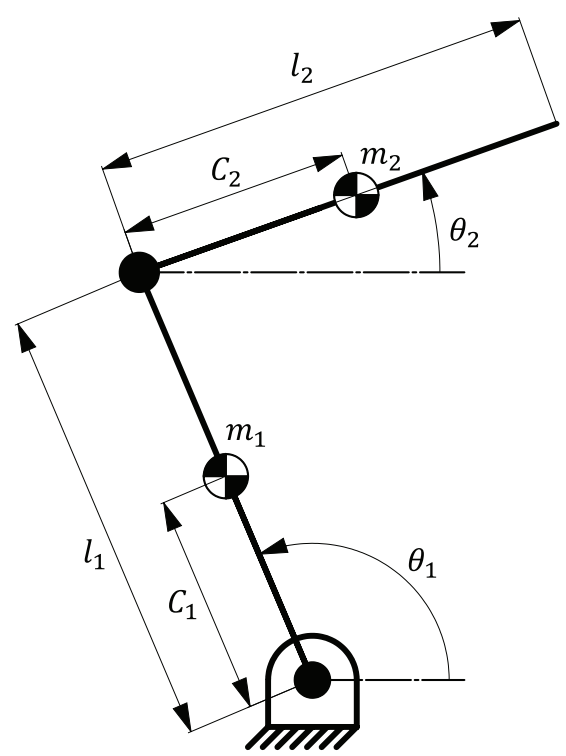

(a)

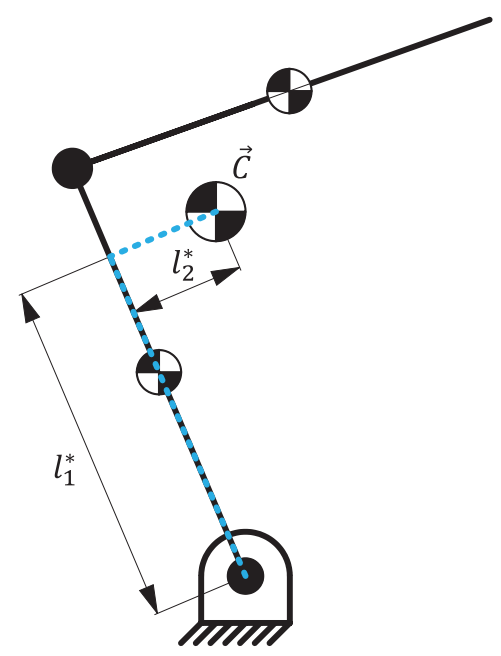

(b)

Figure 1.1: (a) The kinematic and static parameters of a planar serial chain system. (b) The SESC model of the planar serial chain system.

\subsubsection{Frame-Based SESC Modeling for Spatial Case}

The standard for work in SESC modeling is the set of observations found in this subsection. Although not the standard for this work, several important observations are noted from this approach. An example of this modeling is presented in order to make these observations. An articulated system of rigid bodies is depicted in Fig. 1.2, and shown schematically in Fig. 1.3. The position of the 
$\operatorname{CoM}(\vec{C})$ for the system is

$$
\left\{\begin{array}{c}
\vec{C} \\
1
\end{array}\right\}=\frac{m_{1}}{M} T_{1}\left\{\begin{array}{c}
\vec{c}_{1} \\
1
\end{array}\right\}+\frac{m_{2}}{M} T_{1} T_{2}\left\{\begin{array}{c}
\vec{c}_{2} \\
1
\end{array}\right\}+\frac{m_{3}}{M} T_{1} T_{3}\left\{\begin{array}{c}
\vec{c}_{3} \\
1
\end{array}\right\}+\frac{m_{4}}{M} T_{1} T_{3} T_{4}\left\{\begin{array}{c}
\vec{c}_{4} \\
1
\end{array}\right\}
$$

where, $\vec{c}_{i} \in \mathbb{R}^{3}$ is a vector locating the CoM of each body in the local reference frame attached to body $i$, or relative to $T_{i}$. The matrix $T_{i}$ is a $4 \times 4$ homogeneous transform used to relate the reference frames associated with any two bodies in the articulated system and is defined as

$$
T_{i}=\left[\begin{array}{cc}
A_{i} & \overrightarrow{d_{i}} \\
\mathbf{0} & 1
\end{array}\right]
$$

Note that $A_{i} \in \mathrm{SO}(3)$ is a rotation matrix, $\vec{d}_{i} \in \mathbb{R}^{3}$ is a displacement vector, and $\mathbf{0} \in \mathbb{R}^{3}$ is a (row) vector of zeros. Accordingly, the CoM of frame 2 in Fig. 1.3 can be located as

$$
T_{1} T_{2}\left\{\begin{array}{c}
\vec{c}_{2} \\
1
\end{array}\right\}
$$

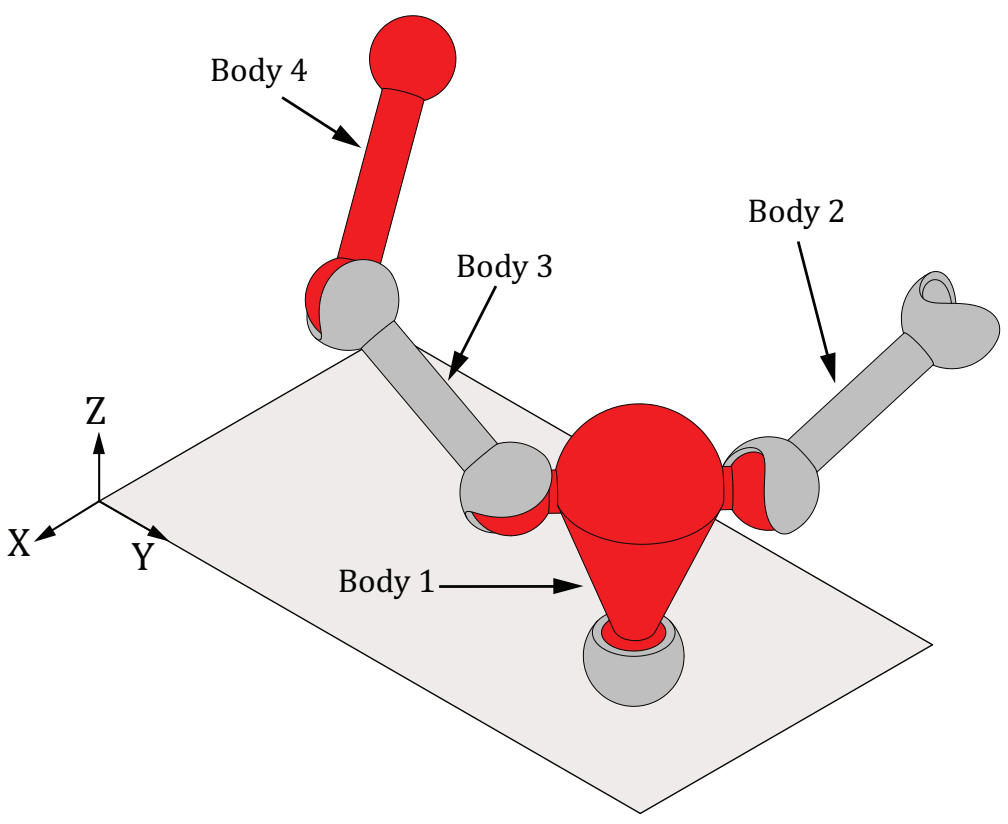

Figure 1.2: An articulated branched spatial system composed of 4 moving rigid bodies. 


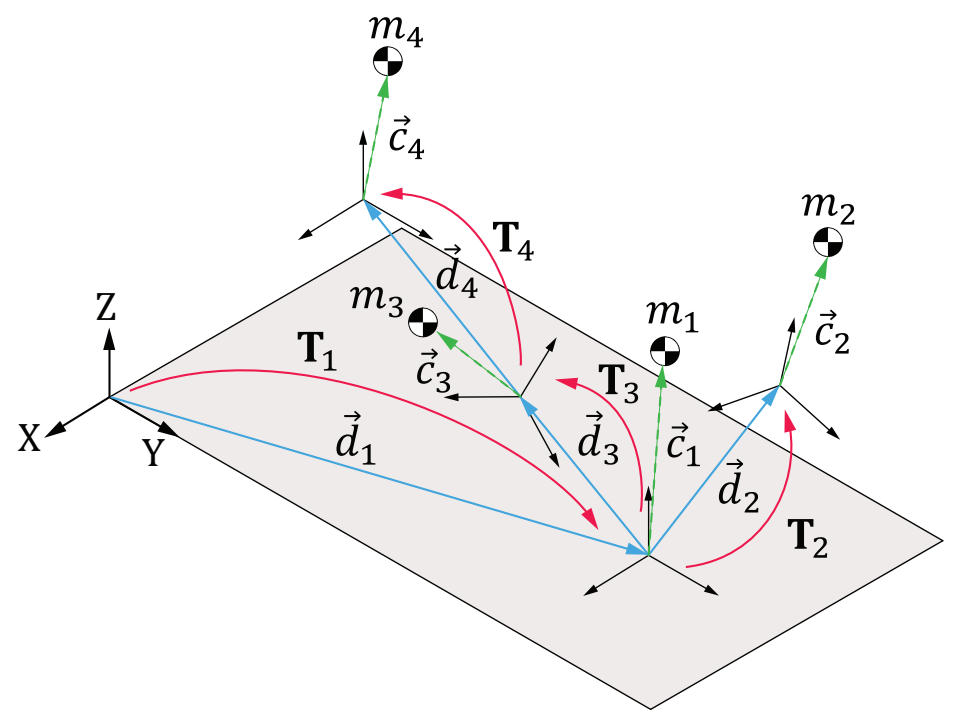

Figure 1.3: The kinematic and static parameters of the spatial branched chain system shown in Fig. 1.2.

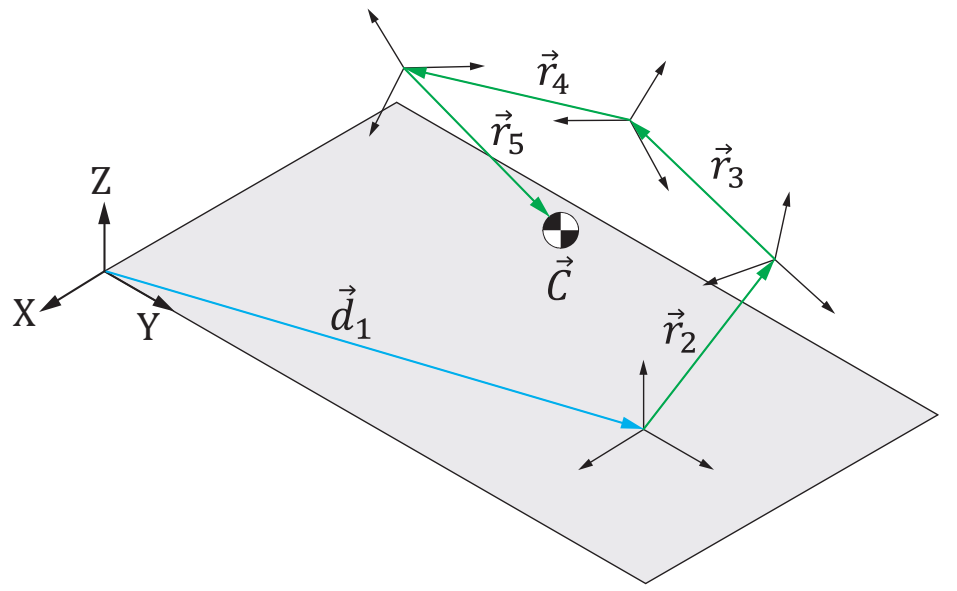

Figure 1.4: The SESC model of the spatial branched chain system shows its terminal point locating the full system's CoM. 
Expanding (1.5) yields

$$
\vec{C}=\vec{d}_{1}+A_{1} \vec{r}_{2}+A_{1} A_{2} \vec{r}_{3}+A_{1} A_{3} \vec{r}_{4}+A_{1} A_{3} A_{4} \vec{r}_{5}
$$

where

$$
\begin{aligned}
& \vec{r}_{2}=\frac{\left(m_{1} \vec{c}_{1}+m_{2} \vec{d}_{2}+\left(m_{3}+m_{4}\right) \vec{d}_{3}\right)}{M}, \quad \vec{r}_{3}=\frac{\left(m_{2} \vec{c}_{2}\right)}{M}, \\
& \vec{r}_{4}=\frac{\left(m_{3} \vec{c}_{3}+m_{4} \vec{d}_{4}\right)}{M}, \quad \vec{r}_{5}=\frac{\left(m_{4} \vec{c}_{4}\right)}{M} .
\end{aligned}
$$

Observe that the $\vec{r}_{i}$ vectors in (1.9) are the SESC vectors and contain the link properties of the original system. If the kinematic and static parameters of the system are known, then the $\vec{r}_{i}$ vector in (1.9) are also known. Furthermore, if the bodies in the system are connected by only revolute (and, hence, universal and spherical joints), the $\vec{d}_{i}$ vectors are constant making the $\vec{r}_{i}$ vectors constant as well. Rewrite (1.8) as

$$
\vec{C}=\vec{d}_{1}+A_{1} \vec{r}_{2}+A_{1} A_{2} \vec{r}_{3}+A_{1} A_{2} \hat{A}_{3} \vec{r}_{4}+A_{1} A_{2} \hat{A}_{3} A_{4} \vec{r}_{5}
$$

where

$$
\hat{A}_{3}=A_{2}^{-1} A_{3} \text {. }
$$

Rearranging (1.10),

$$
\left\{\begin{array}{c}
\vec{C} \\
1
\end{array}\right\}=\left[\begin{array}{cc}
A_{1} & \vec{d}_{1} \\
\mathbf{0} & 1
\end{array}\right]\left[\begin{array}{cc}
A_{2} & \vec{r}_{2} \\
\mathbf{0} & 1
\end{array}\right]\left[\begin{array}{cc}
\hat{A}_{3} & \vec{r}_{3} \\
\mathbf{0} & 1
\end{array}\right]\left[\begin{array}{cc}
A_{4} & \vec{r}_{4} \\
\mathbf{0} & 1
\end{array}\right]\left\{\begin{array}{c}
\vec{r}_{5} \\
1
\end{array}\right\}
$$

Observe that the expression in (1.12) is similar to the forward kinematics of a serial chain. As a result, the position of the CoM of the original branched chain is modeled by the terminal point of a virtual spatial serial chain of appropriate size as depicted by the green arrows in Fig. 1.4 where the terminal point is the CoM. Note that the SESC has the same number of degrees of freedom as the original branched spatial chain. Thus, the motion of its joints correspond to those of the original system. Equation (1.12), for a branched spatial chain, is analogous to the system in Fig. 1.2 in the 
same way as (1.3) is to the two-body planar system in Fig. 1.1a. As the system moves, the SESC behaves like a virtual serial chain that terminates at the system's CoM.

\subsection{Movement Analysis Systems}

The late 19th century witnessed the recording of locomotion patterns, for both humans and animals, using the first motion picture cameras [40]. Muybridge [41] illustrated in 1877, with photographs, horse locomotion by showing that, while the horse is running fast, there is a moment in time where all of the animal's feet are off the ground. In 1901 he extended the research to humans by using the same motion cameras to study the movement patterns of a running man [42]. In 1882 Marey [43] used a photographic rifle to record human gait displacement in order to produce a stick figure of a runner. Movement analysis systems are used to reconstruct two or three dimensional movement data either by using a single camera or multiple cameras, allowing quantification of the kinematics of different gaits. In 1980 Woltring [44] showed that as the number of cameras increased the error in the three dimensional coordinates decreased. VICON, a marker-based motion camera, became commercially available in 1982 [45]. Other similar systems have followed including the Motion Analysis Corporation system [46] and Qualisys [47]. The primary, though not only, equipment used for data acquisition in the experimental construction of the SESC are the Xbox Kinect and the Wii Balance Board, Fig. 1.5. The technique is not limited to these sensors and can be applied using other available sensors introduced in the following subsections.

\subsubsection{Marker-Based Motion Capture Systems}

To detect subject motion, marker-based motion capture relies on markers placed on anatomical landmarks to represent the body segments as shown in Fig. 1.7b. These markers are classified into two types, passive markers (Fig. 1.6a) and active markers (Fig. 1.6b). Passive markers are generally

made from a spherical retro-reflective material known as Scotchlite [40], which reflects the light 


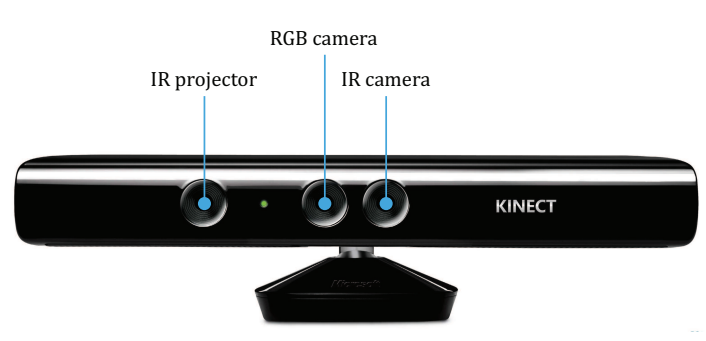

(a)

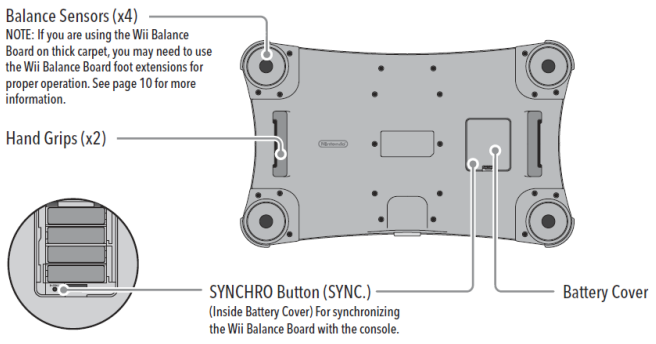

(b)

Figure 1.5: (a) Kinect sensor components including the IR projector, the RGB camera, and the IR camera [1]. (b) WBB sensor components showing the 4 pressure sensors (strain gauges) at each corner [2].

emitted from the camera back to the camera lens. The VICON system (Oxford Metric Group) is one of the most common systems that uses passive markers.

Conversely, active markers emit a light pattern at a given frequency and do not depend on illumination allowing them to be easily identified and tracked. The ability of active markers to have their own frequency allows them to be automatically detected without being misidentified with adjacent markers as can happen with passive ones. Another advantage over passive markers is their use outdoors, unlike passive markers which are confined to indoor use due to their sensitivity to light. An example of a common system that implements active markers is the Optotrak (NDI Measurement Sciences) [4].

\subsubsection{Markerless Motion Capture Systems}

Markerless movement sensors utilize computer vision technology to detect and track subjects, requiring no markers to provide information to the image processing software as shown in Fig. 1.7a. These systems rely on advanced algorithms to accurately track real-time motions. The only research grade markerless motion capture sensor that is currently commercially available is OpenStage 2 (Organic Motion). As such, markerless motion capture remains an area of active research [48]. 


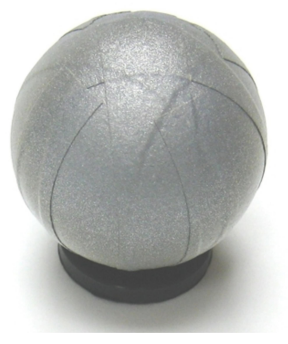

(a)

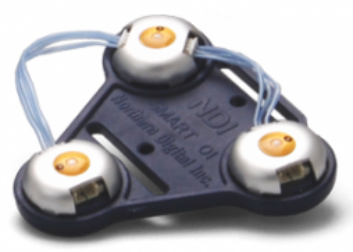

(b)

Figure 1.6: (a) The VICON passive marker is made from a spherical retro-reflective material capable of reflecting the light emitted from the camera back to the camera lens [3]. (b) The Optotrak active marker can emit its own light pattern at a given frequency and does not depend on illumination [4].

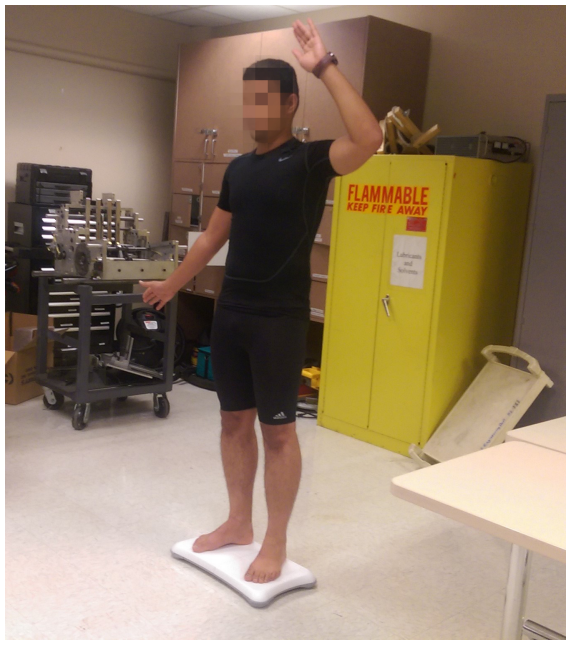

(a)

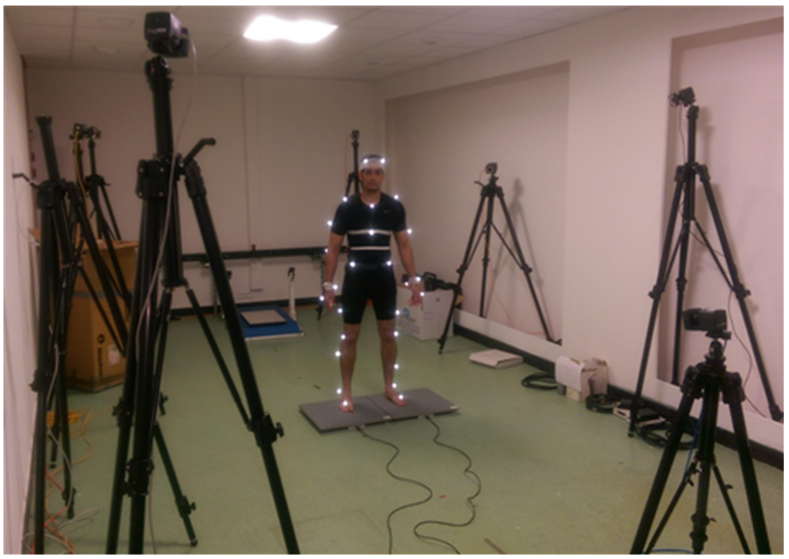

(b)

Figure 1.7: (a) A markerless based motion capture system does not require the use of markers for motion detection and tracking. (b) A marker-based motion capture system relies on the markers for motion detection and tracking. 
The most affordable markerless sensor is from Microsoft. The Kinect was introduced as a gaming product allowing consumers to interact with videogames simply by moving their bodies. The Kinect sensor consists of an RGB camera, an IR projector, and an IR camera as shown in Fig. 1.5a. The depth sensor is composed of the IR projector combined with the IR camera, which is a monochrome complementary metal-oxide semiconductor (CMOS) sensor $[49,50]$. The IR emitter of the Kinect sensor projects a pattern of infrared light of varying intensities. The IR camera reconstructs the depth image by recognizing the distortion of this pattern of infrared light. Kinect allows real time 3D motion tracking using a skeletal model capable of tracking 20 nodes as depicted in Fig. 3.1a. Clark et al. [51] showed that the Kinect combined with the Microsoft SDK are capable of providing comparable data to VICON when assessing anatomical landmark position and angular displacement data during postural control. Stepan et al. [52] showed that as the subject turns away from the Kinect the joint estimation fails and the performance of the Kinect decreases. However, in a controlled body posture like standing and exercising the arms, the joint estimation has been shown to be comparable to those provided by VICON and PhaseSpace motion capture systems. To implement the Kinect, the Microsoft SDK [1], Microsoft Visual Studio [53], and the C-sharp programming language [54] are used in the data collection process.

\subsubsection{Force Platforms}

Force plates are classified into two categories, using either strain gauges or piezoelectrics. Both systems are capable of measuring and recording the ground reaction forces and their point of action (center of pressure, $\mathrm{CoP}$ ). The ground reaction force consists of three components acting at the CoP. These forces are the vertical forces represented by the weight of the body and its progression over the supporting limb, the anterior-posterior forces as the accelerating and breaking forces, and the medial-lateral forces as the side to side acting force. 
Piezoelectric force plates are more sensitive than those with strain gauges and allow a larger range of force measurements. Generally, piezoelectric force plates are more expensive.

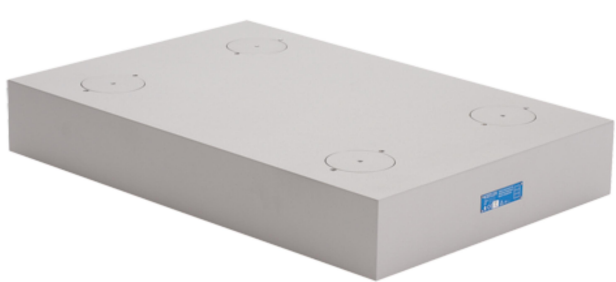

(a)

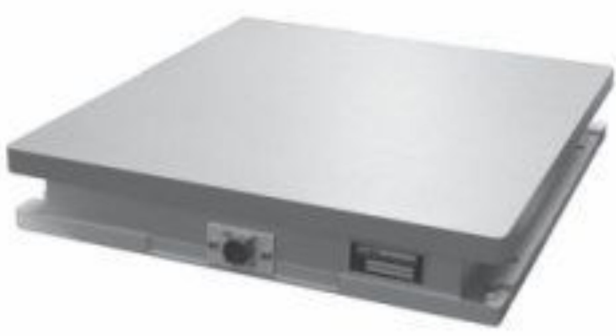

(b)

Figure 1.8: (a) Kistler-9281E, a piezoelectric force plate [5] and (b) AMTI-OR6-7, a strain gauge force plate [6].

\subsubsection{The Wii Balance Board}

The Nintendo Wii balance board is equipped with four pressure sensors (strain gauges) at each corner as illustrated in Fig. 1.5b. By utilizing the forces measured at these locations, the CoP position and the vertical component of the ground reaction force may be approximated. Many studies utilizing the WBB in rehabilitation $[55,56]$ show encouraging results when compared to laboratory-grade force plates [57]. In order to connect to and read the data from the WBB, the open source .NET managed library WiimoteLib was utilized [58].

\subsection{Center of Pressure and Center of Mass}

Force plates measure center of pressure and, if the subject on the force plate is static, the projection of the center of mass. This section clarifies the relationship between the $\operatorname{CoP}\left(\vec{C}_{p}\right)$ and the $\operatorname{CoM}$ projection $\left(\vec{C}_{W}\right)$, and justifies their use in the experimental portion of this work. The horizontal 
component of the $\mathrm{CoM}$ is referred to as the $\mathrm{CoM}$ projection. The $\mathrm{CoP}$ is the location of the vertical ground reaction force and is the weighted average of all the pressures over the surface of the area in contact with the ground.

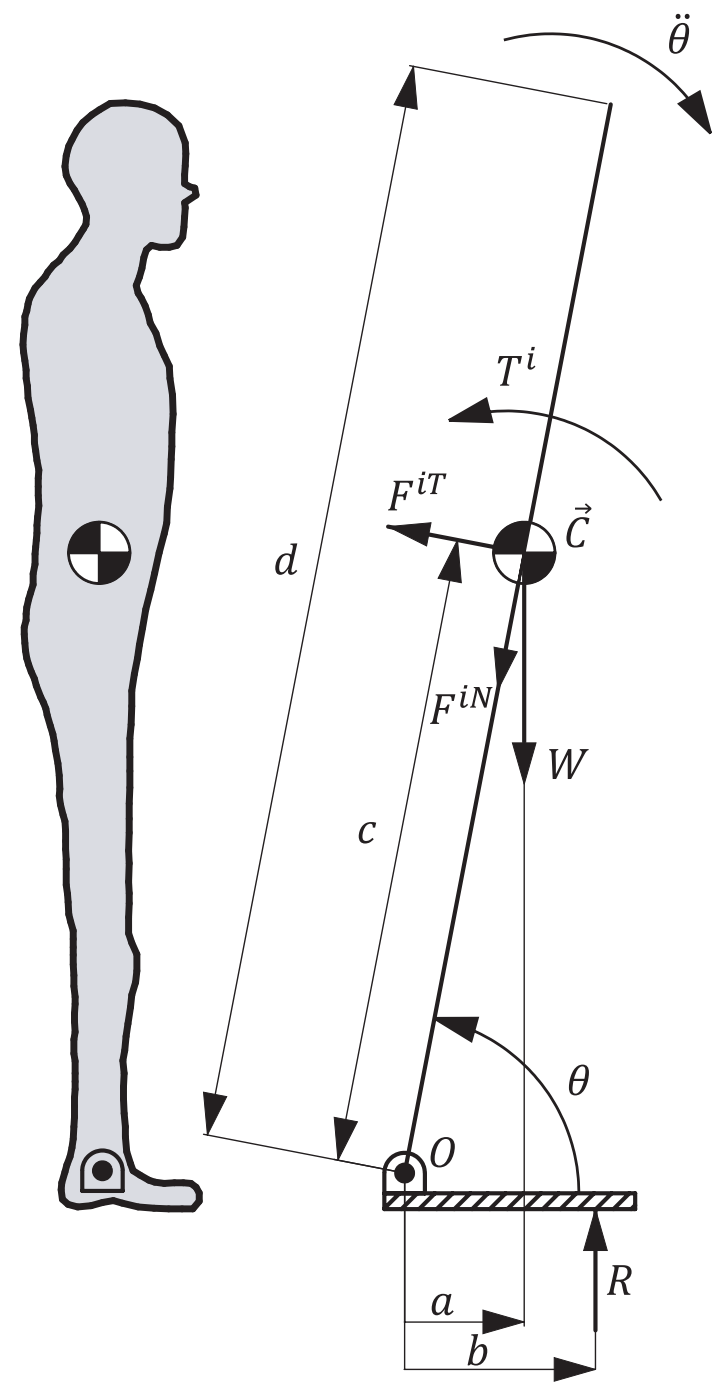

Figure 1.9: A model of a human as an inverted pendulum which includes: the CoM $(\vec{C})$, the COP position $(b)$, the CoM projection position $(a)$, the body weight $(W)$, the tangent vector of the inertial force $\left(F^{i T}\right)$, the normal vector of the inertial force $\left(F^{i N}\right)$, the inertial torque $\left(T^{i}\right)$, the height of CoM $(c)$, the height of the inverted pendulum $(d)$, and the ground reaction force $(R)$. 


\subsubsection{Human Balance}

Balance control during quiet standing is frequently modeled as a planar (2D) inverted pendulum $[9,59,60]$ as depicted in Fig. 1.9. This model assumes the feet are side-by-side and the ankle rotations are aligned. Thus, the body is an inverted pendulum that pivots about the ankle joint. In Fig. 1.9 the body weight $(W)$ is opposite to the vertical ground reaction force $(R)$ and these forces act at distance $a$ and $b$ respectively from the fixed pivot point $O$. Applying D'Alembert's principle, summing moments around pivot $O$ and forces along the $y$ direction, yields

$$
\begin{aligned}
& \sum M_{O}=T^{i}+F^{i T} c-W a+R b=0, \\
& \sum F_{y}=R-W+F^{i T} \cos \theta-F^{i N} \sin \theta=0,
\end{aligned}
$$

where

$$
\begin{aligned}
& T^{i}=I_{G} \ddot{\theta}, \\
& F^{i T}=m c \ddot{\theta}, \\
& F^{i N}=m c \dot{\theta}^{2} .
\end{aligned}
$$

Observe that the summation of forces in the $x$ direction is balanced by the friction force and is not needed for this analysis. The parameter $I_{G}$ is the moment of inertia of the total body about the

pivot point $O, \ddot{\theta}$ is the angular acceleration of the inverted pendulum, $\dot{\theta}$ is the angular velocity of the inverted pendulum, $m$ is the mass of the body and $c$ is the distance from the pivot point $O$ to the CoM. Noting that $a=c \cos \theta$, and substituting (1.16) and (1.17) into (1.14),

$$
R-W+m c \ddot{\theta} \cos \theta-m c \dot{\theta^{2}} \sin \theta=0 .
$$

Ideally, the body experiences no sway in a static posture and the angular velocity and acceleration approach zero, $\dot{\theta}=\ddot{\theta}=0$, simplifying (1.18) to

$$
R=W
$$


Thus, in quiet standing the body weight and the vertical ground reaction force are equal and $R$ remains constant. Substituting (1.15) and (1.16) into (1.13),

$$
\ddot{\theta}\left(I_{G}+m c^{2}\right)=W a-R b .
$$

From (1.19), $W=R$ for static postures, and (1.20) simplifies to

$$
\ddot{\theta} K=a-b
$$

where

$$
K=\frac{I_{G}+m c^{2}}{W}
$$

is a constant. From (1.21), observe that the difference between the $\mathrm{CoP}$ and the CoM projection is proportional to the angular acceleration of the inverted pendulum. If the body experiences no sway, the angular acceleration will approach zero and (1.21) simplifies to

$$
a=b .
$$

Therefore, relying solely on the CoP data to estimate the CoM can be problematic. Observing both the MoCap and the force plate data simultaneously, both need to indicate low accelerations and velocities to obtain an accurate estimate for the CoM. This state is termed being nearly static. The concept of being nearly static during experimentation is quantified in Chapter IV.

\subsection{Summary}

The remainder of the dissertation is arranged as follows. Chapter II presents the force plate and motion capture parameter identification, detailing the experimental protocol and the numerical procedure used to estimate the orientation and displacement of the force plate relative to the MoCap system. Chapter III introduces the node-based Statically Equivalent Serial Chain method and details the modeling process and the theoretical aspects of the formulation when the relative 
orientation and displacement of the force plate are either known or unknown. The formulation of the node-based SESC donor model is then introduced. The donor model relies on a donor subject by utilizing an existing set of SESC parameters to estimate a new set of parameters capable of estimating a new subject's CoM. In the case of subjects with limited body motion, the donor model requires fewer postures to generate an accurate estimate of the subject's CoM. The extension to the node-based SESC modeling technique to include an extra static body to the workspace is presented. The inclusion of a static body aids in balance and stability and allow for diverse postures. A further extension to node-based SESC modeling allowing an arbitrary location of the CoM of an individual body is presented. This is needed due to assumptions made about individual body CoM locations potentially being over-restrictive. Chapter IV identifies and experimentally validates the node-based SESC techniques for several human subjects. The chapter provides experimental results from the low-end (Kinect/WBB) and high-end (VICON/AMTI-OR6) sensors. Chapter V concludes the dissertation by highlighting and summarizing the contributions of this work. Future research is also presented. 


\section{CHAPTER II}

\section{FORCE PLATE AND MOTION CAPTURE PARAMETER IDENTIFICATION}

The WBB and the Kinect have separate frames of reference. In order for the two sensors to communicate with each other, the relative orientation of the two frames (A) and the displacement

$(\vec{d})$ need to be obtained. This section will introduce a method for estimating $\mathbf{A}$ and $\vec{d}$. Note that these parameters can be constructed from the data collected during the experiment and is a process discussed in Chapter III. Although higher end systems include calibration tools for this operation, in the case of the WBB and the Kinect this development served as part of the research work.

\subsection{Experiment Protocol}

The separate frames of reference of the Kinect and the WBB are shown in Fig. 2.1. However, to align with convention without loss of generality, the two frames are arranged as show in Fig. 2.2. Accordingly, the rotation that relates collected data from Kinect to that used in the procedure is,

$$
\left[\begin{array}{lll}
0 & 0 & 1 \\
1 & 0 & 0 \\
0 & 1 & 0
\end{array}\right],
$$

while the rotation that relates collected data from WBB to that used in the procedure is,

$$
\left[\begin{array}{ccc}
0 & 1 & 0 \\
1 & 0 & 0 \\
0 & 0 & -1
\end{array}\right] .
$$

The experiment starts by placing the Kinect sensor on a flat, stable surface. The weight is then positioned such that the front face of the target is facing the Kinect as shown in Fig. 2.3. The weight 
used is $30 \mathrm{~kg}$ and rotationally symmetric so that the horizontal location of its CoM could be readily determined. The square target is attached to the top surface of the weight, directly over its CoM. The weight is then moved to several positions across the surface of the WBB as shown in Fig. 2.4. At each position the target is located on the software screen and the location and CoP are recorded by clicking on the target.

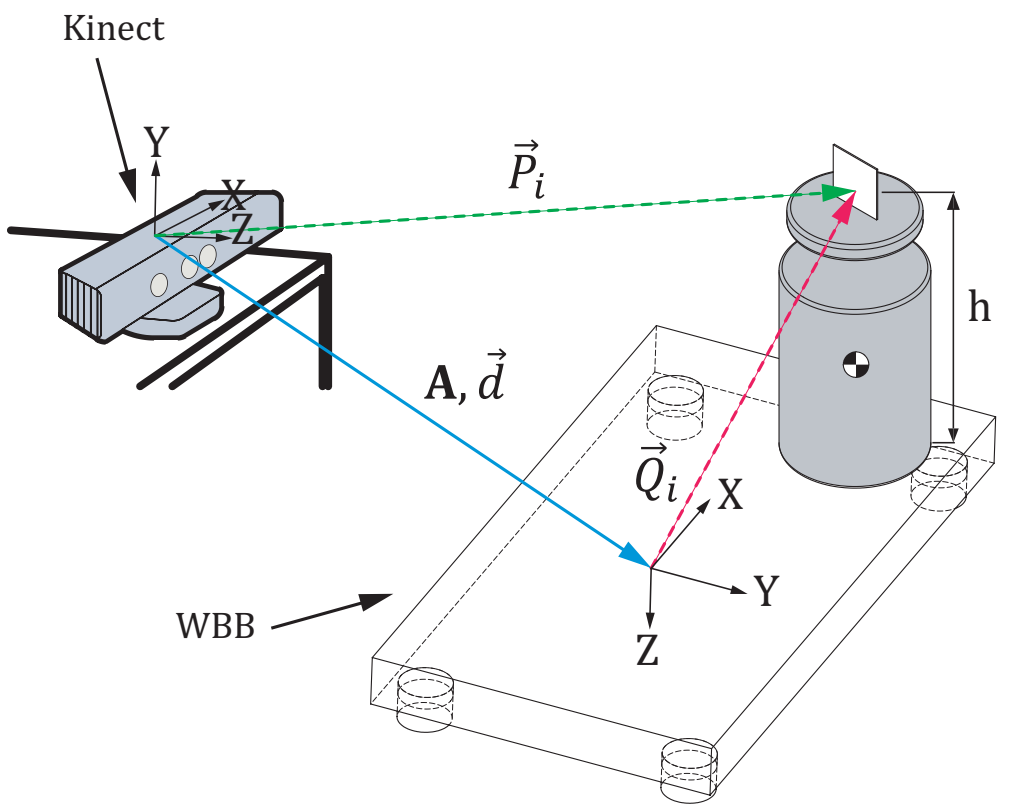

Figure 2.1: The actual orientation of the Kinect and the WBB reference frames. The Kinect's reference frame has the $z$ axis pointing toward the user and the WBB's reference frame has the $z$ axis pointing downward matching the direction of gravity.

\subsection{The Estimation of A and $\vec{d}$ Relative to The MoCap}

Given the data collected above, the following formulation shows the determination of $\mathbf{A}$ and $\vec{d}$. The $i^{\text {th }}$ position of the target relative to the Kinect, depicted in Fig. 2.2, is

$$
\vec{P}_{i}, \quad i=1, \ldots, n
$$




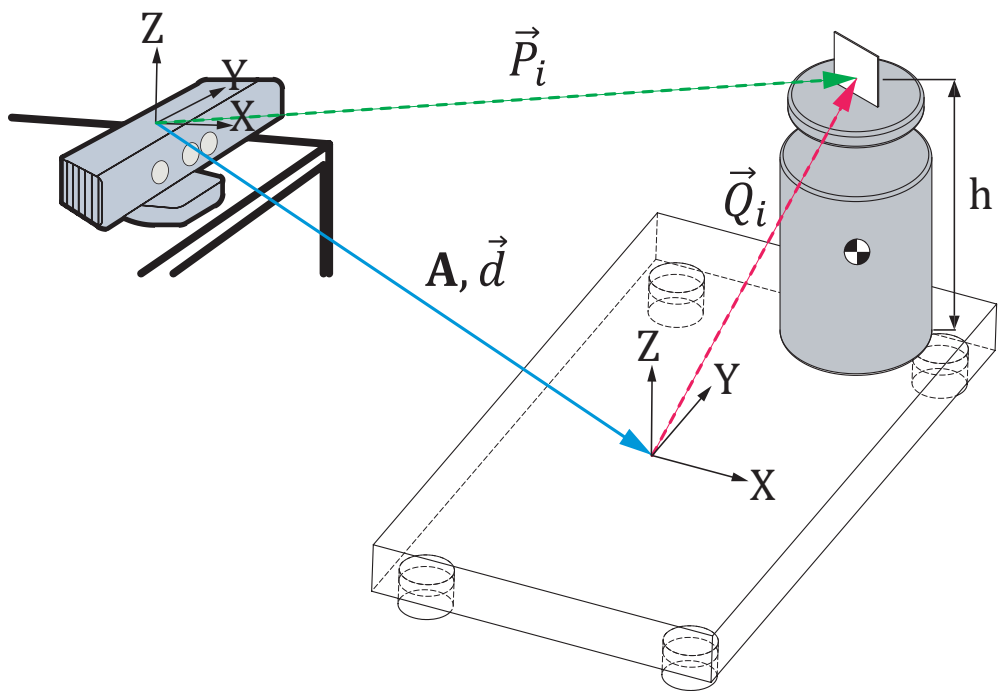

Figure 2.2: The experimental setup where the target weight is placed on the top of the WBB facing the Kinect. The kinematic and static parameters of the target weight are shown.

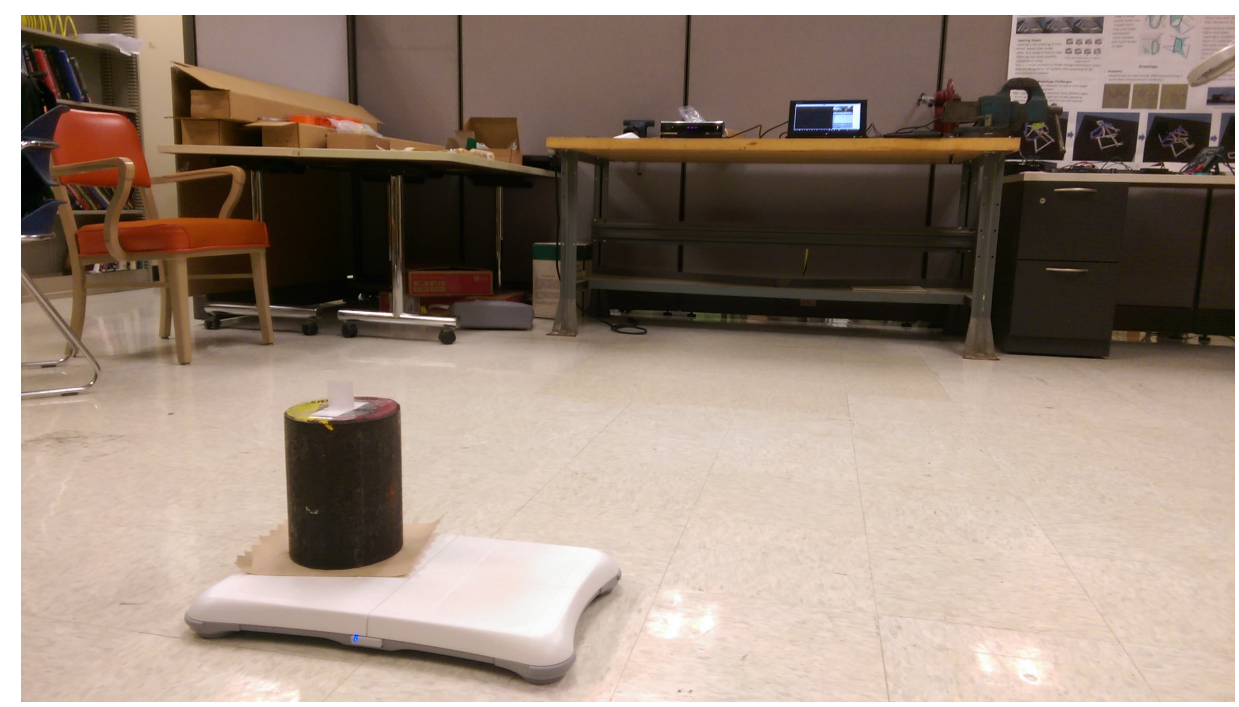

Figure 2.3: The parameter identification experiment to determine $\mathbf{A}$ and $\vec{d}$ includes the WBB, the Kinect and the target weight. 


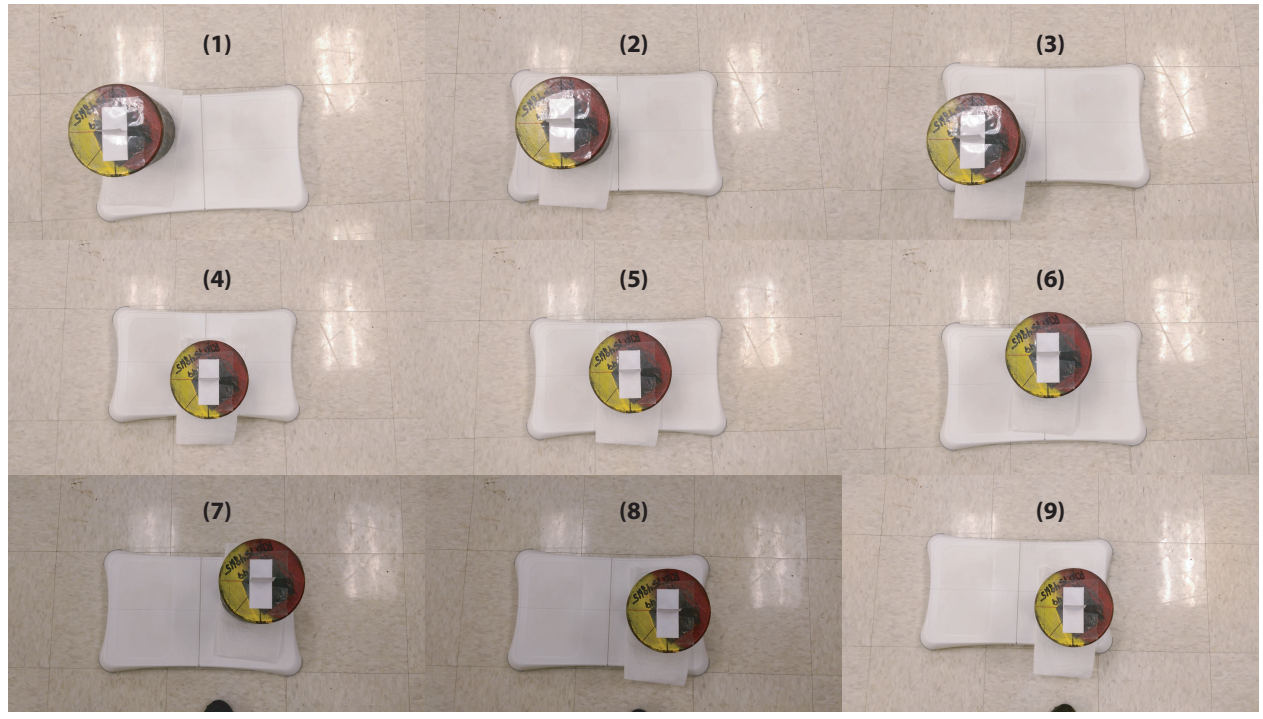

Figure 2.4: Top view of the target weight as seen on the WBB, during the parameter identification experiment, in 9 different positions to record its location via the Kinect and WBB.

The location of the target on the WBB is

$$
\vec{Q}_{i}=\left\{\begin{array}{c}
q_{x_{i}} \\
q_{y_{i}} \\
h
\end{array}\right\},
$$

where $h$ is a known, fixed height and $q_{x_{i}}$ and $q_{y_{i}}$ are the center of pressure readings from the WBB. For static conditions, they are directly under the CoM. Equating (2.3) and (2.4),

$$
\vec{P}_{i}=\mathbf{A} \vec{Q}_{i}+\vec{d}
$$

Using (2.5) with two experimental positions, $i$ and $i+1$ from the Kinect and the WBB, the difference of two instances is

$$
\delta \vec{P}_{j}=\mathbf{A} \delta \vec{Q}_{j}, \quad j=1, \ldots, n
$$

where $j$ is the $j^{\text {th }}$ difference of two data points. Under the assumption that the $z$ axes align, the $z$ component is 0 for $\delta \vec{P}_{j}$ and $\delta \vec{Q}_{j}$. Acquiring $n$ instances of (2.6) yields

$$
\left[\begin{array}{llll}
\delta \vec{P}_{1} & \delta \vec{P}_{2} & \ldots & \delta \vec{P}_{n}
\end{array}\right]=\mathbf{A}\left[\begin{array}{llll}
\delta \vec{Q}_{1} & \delta \vec{Q}_{2} & \ldots & \delta \vec{Q}_{n}
\end{array}\right]
$$


represented as

$$
\mathbf{M}=\mathbf{A F}
$$

where $\mathbf{M}$ and $\mathbf{F} \in \mathbb{R}^{3 \times n}$. Via the pseudo-inverse,

$$
\mathbf{M F}^{+}=\left[\begin{array}{lll}
x_{1} & x_{2} & 0 \\
y_{1} & y_{2} & 0 \\
z_{1} & z_{2} & 0
\end{array}\right]=\left[\begin{array}{lll}
\vec{X} & \vec{Y} & \overrightarrow{0}
\end{array}\right],
$$

observe that the third column is identically 0 and does not identify a suitable A matrix. However, the third column can be established as $\vec{Z}=\vec{X} \times \vec{Y}$, and the three vectors combine to form an approximation of $\mathbf{A}$ as

$$
\hat{\mathbf{A}}=\left[\begin{array}{lll}
\vec{X} & \vec{Y} & \vec{Z}
\end{array}\right]=\mathbf{U} \mathbf{\Sigma} \mathbf{V}^{\mathbf{T}}
$$

where $\mathbf{U} \boldsymbol{\Sigma} \mathbf{V}^{\mathbf{T}}$ is the singular value decomposition. The value of $\mathbf{A}$ is [61]

$$
\mathbf{A}=\mathbf{U V}^{\mathbf{T}}
$$

With A established, $\vec{d}$ can now be approximated. Rearranging (2.5) and acquiring enough instances yields

$$
\left[\begin{array}{cccc}
\vec{d}_{1}^{*} & \overrightarrow{d_{2}^{*}} & \ldots & \vec{d}_{n}^{*}
\end{array}\right]=\left[\begin{array}{llll}
\vec{P}_{1} & \vec{P}_{2} & \ldots & \vec{P}_{n}
\end{array}\right]-\mathbf{A}\left[\begin{array}{llll}
\vec{Q}_{1} & \vec{Q}_{2} & \ldots & \vec{Q}_{n}
\end{array}\right]
$$

In theory, the $\overrightarrow{d_{i}^{*}}$ are equal. Thus, the displacement vector is given by

$$
\vec{d}=\frac{1}{n} \sum_{i=1}^{n} \overrightarrow{d_{i}^{*}}
$$




\section{CHAPTER III}

\section{NODE-BASED STATICALLY EQUIVALENT SERIAL CHAIN}

The center of mass (CoM) of an articulated system of rigid bodies can be expressed as the terminal point of a virtual serial chain. Such a chain is termed the statically equivalent serial chain (SESC). The node-based SESC method is now formulated, a methodology based on the node positions defining a skeletal frame. This is desirable due to the information generated by markerless MoCap systems in general, and the Kinect specifically.

\subsection{Human Skeleton Model}

The Kinect sensor produces a skeletal model of 19 links as seen in Fig. 3.1a. However, the contribution of several links of the chain to the overall CoM are negligible due to insignificant masses. Additionally, in the case of Kinect the distal links (hands and feet), for example, have a higher variation in distances between the nodes [52]. Thus, the skeleton model was reduced to 13 links.

\subsection{Model Construction of a Human Subject where A and $\vec{d}$ are Known}

The node-based SESC theory is presented here via the analysis of a human modeled as 13 rigid links connected by spherical joints. Consistent with [17, 62], the CoM position for each of the links is assumed to lie along the line defined by the nodes at successive joints as shown in Fig. 3.2. 


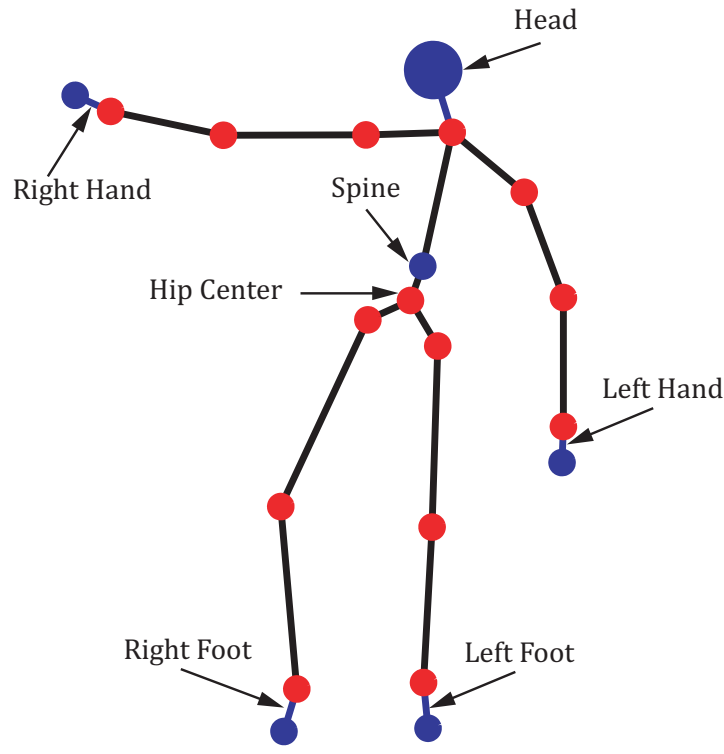

(a)

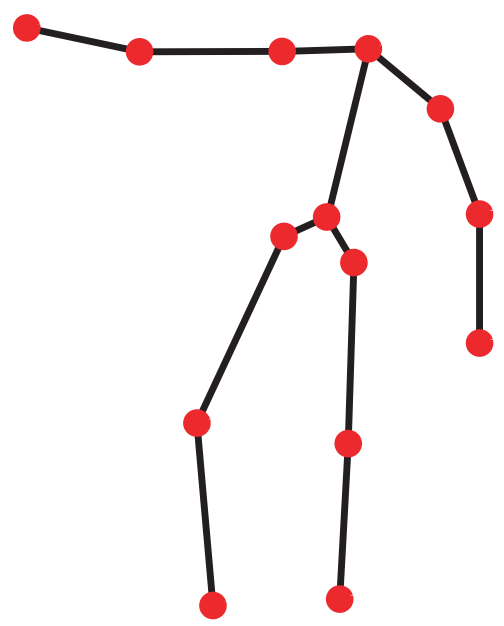

(b)

Figure 3.1: (a) The skeletal model generated by the Kinect sensor is composed of 19 links. Note that the links and nodes in blue are neglected. (b) The reduced human skeleton model is composed of 13 links.

The node-based SESC model can be constructed either with the knowledge of the relative location of the force-plate to the MoCap system (via the process introduced in Chapter II) or without the knowledge of the relative location.

To estimate the CoM of human subjects, the subject will perform several static postures. The derivation in this section assumes that $\mathbf{A}$ and $\vec{d}$, the location of the force plate relative to the MoCap, were found using the method described in Chapter II. The $j^{\text {th }}$ position of the CoM $(\vec{C})$ of the articulated system of 13 rigid bodies, when striking a static posture, is depicted in Fig. 3.2 and can 
be defined as

$$
\begin{aligned}
M \vec{C}_{j}= & m_{1}\left(\vec{P}_{j}+\lambda_{1} \vec{\tau}_{1_{j}}\right)+m_{2}\left(\vec{P}_{j}+\vec{\tau}_{1_{j}}+\lambda_{2} \vec{\tau}_{2_{j}}\right)+m_{3}\left(\vec{P}_{j}+\vec{\tau}_{1_{j}}+\vec{\tau}_{2_{j}}+\lambda_{3} \vec{\tau}_{3_{j}}\right) \\
& +m_{4}\left(\vec{P}_{j}+\lambda_{4} \vec{\tau}_{4_{j}}\right)+m_{5}\left(\vec{P}_{j}+\vec{\tau}_{4_{j}}+\lambda_{5} \vec{\tau}_{5_{j}}\right)+m_{6}\left(\vec{P}_{j}+\vec{\tau}_{4_{j}}+\vec{\tau}_{5_{j}}+\lambda_{6} \vec{\tau}_{6_{j}}\right) \\
& +m_{7}\left(\vec{P}_{j}+\lambda_{7} \vec{\tau}_{7_{j}}\right)+m_{8}\left(\vec{P}_{j}+\vec{\tau}_{7_{j}}+\lambda_{8} \vec{\tau}_{8_{j}}\right)+m_{9}\left(\vec{P}_{j}+\vec{\tau}_{7_{j}}+\vec{\tau}_{8_{j}}+\lambda_{9} \vec{\tau}_{9_{j}}\right) \\
& +m_{10}\left(\vec{P}_{j}+\vec{\tau}_{7_{j}}+\vec{\tau}_{8_{j}}+\vec{\tau}_{9_{j}}+\lambda_{10} \vec{\tau}_{10_{j}}\right)+m_{11}\left(\vec{P}_{j}+\vec{\tau}_{7_{j}}+\lambda_{11} \vec{\tau}_{11_{j}}\right) \\
& +m_{12}\left(\vec{P}_{j}+\vec{\tau}_{7_{j}}+\vec{\tau}_{11_{j}}+\lambda_{12} \vec{\tau}_{12_{j}}\right) \\
& +m_{13}\left(\vec{P}_{j}+\vec{\tau}_{7_{j}}+\vec{\tau}_{11_{j}}+\vec{\tau}_{12_{j}}+\lambda_{13} \vec{\tau}_{13_{j}}\right),
\end{aligned}
$$

where $M$ is the total mass of the human subject, $m_{i}$ is the mass of body $i, \lambda_{i}$ is a scalar, $\vec{P}_{j} \in \mathbb{R}^{3}$ is a position vector of one of the system joints (nodes) in the MoCap reference frame as depicted in Fig. 3.2, and $\vec{\tau}_{i_{j}} \in \mathbb{R}^{3}$ is a vector between the nodes defining body $i$. Grouping terms,

$$
\vec{C}_{j}=\vec{P}_{j}+\sum_{i=1}^{13} \vec{\tau}_{i_{j}} s_{i},
$$

where, $s_{1}$ through $s_{13}$ are termed the SESC parameters:

$$
\begin{aligned}
& s_{1}=\frac{m_{1} \lambda_{1}+m_{2}+m_{3}}{M}, \quad s_{2}=\frac{m_{2} \lambda_{2}+m_{3}}{M}, \quad s_{3}=\frac{m_{3} \lambda_{3}}{M}, \quad s_{4}=\frac{m_{4} \lambda_{4}+m_{5}+m_{6}}{M}, \\
& s_{5}=\frac{m_{5} \lambda_{5}+m_{6}}{M}, \quad s_{6}=\frac{m_{6} \lambda_{6}}{M}, \quad s_{7}=\frac{m_{7} \lambda_{7}+m_{8}+m_{9}+m_{10}+m_{11}+m_{12}+m_{13}}{M}, \\
& s_{8}=\frac{m_{8} \lambda_{8}+m_{9}+m_{10}}{M}, \quad s_{9}=\frac{m_{9} \lambda_{9}+m_{10}}{M}, \quad s_{10}=\frac{m_{10} \lambda_{10}}{M}, \\
& s_{11}=\frac{m_{11} \lambda_{11}+m_{12}+m_{13}}{M}, \quad s_{12}=\frac{m_{12} \lambda_{12}+m_{13}}{M}, \quad s_{13}=\frac{m_{13} \lambda_{13}}{M} .
\end{aligned}
$$

The SESC parameters contain the kinematic and static parameters of the original system. Note that $\vec{P}_{j}$ may be an arbitrarily selected node and that (3.1)-(3.3) may be readily reformulated relative to any other node. Examining (3.2),

$$
\vec{C}_{j}=\vec{P}_{j}+\left[\begin{array}{llll}
\vec{\tau}_{1_{j}} & \vec{\tau}_{2_{j}} & \ldots & \vec{\tau}_{13_{j}}
\end{array}\right]\left\{\begin{array}{c}
s_{1} \\
s_{2} \\
\vdots \\
s_{13}
\end{array}\right\}=\vec{P}_{j}+\mathbf{T}_{j} \vec{S}
$$




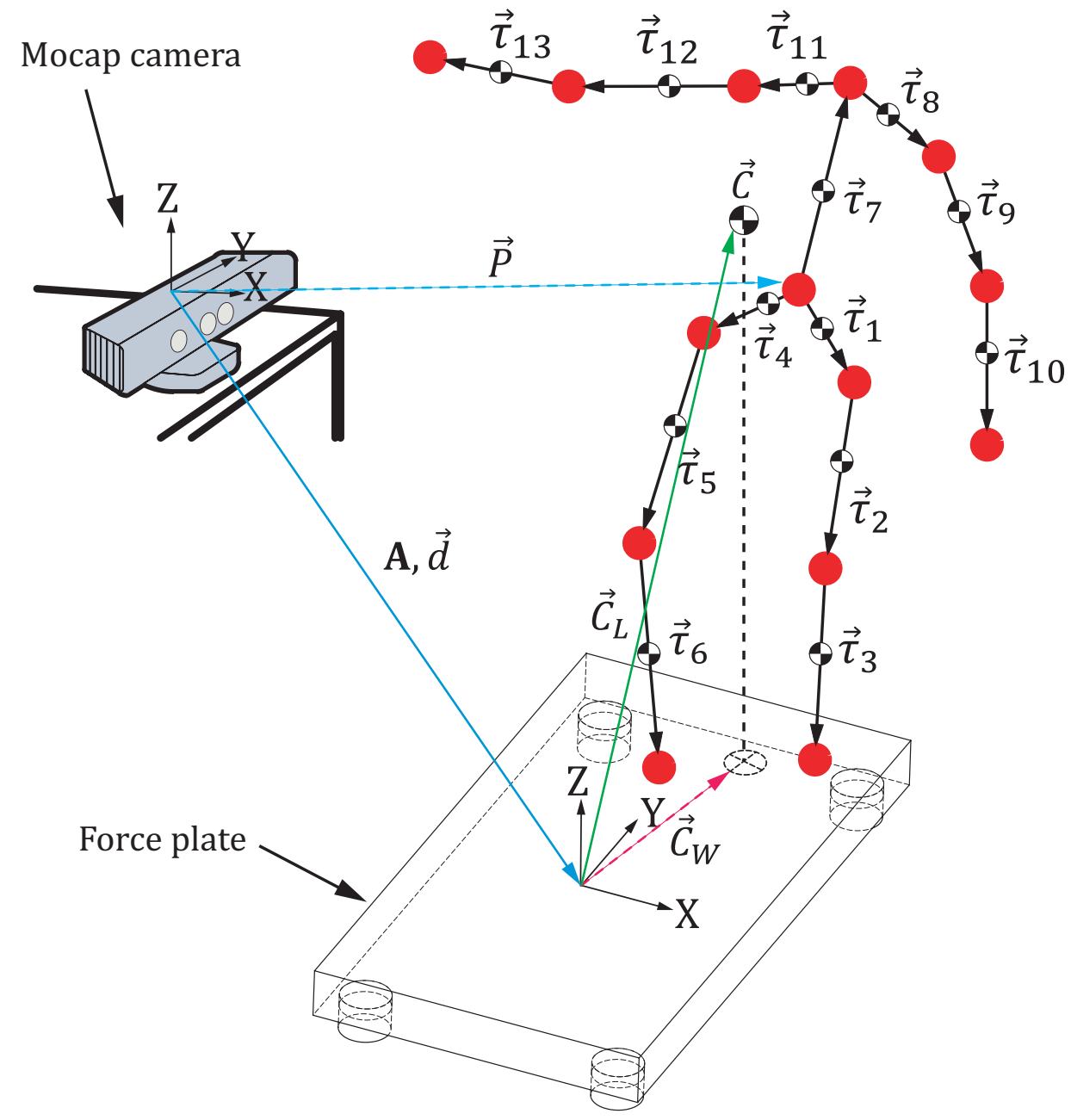

Figure 3.2: The skeleton model of a human subject composed of 13 rigid links. The kinematic and static parameters of the subject are shown. 
where $\vec{S} \in \mathbb{R}^{13}$ contains the SESC parameters and $\mathbf{T}_{j} \in \mathbb{R}^{3 \times 13}$ contains the relative node locations $\vec{\tau}_{1_{j}}$ through $\vec{\tau}_{13_{j}}$. The values of the $\vec{\tau}_{i_{j}}$ correspond to the links (rigid bodies) and their lengths at positions $j$ and $j+1$ are equal neglecting experimental error (i.e. $\left|\vec{\tau}_{1_{j}}\right|=\left|\vec{\tau}_{1_{j+1}}\right|$ ). Noting that the force plate and MoCap have separate frames of reference, as illustrated in Fig. 3.2,

$$
\vec{C}_{j}=\mathbf{A} \vec{C}_{L_{j}}+\vec{d}
$$

Ideally,

$$
\vec{C}_{j}=\mathbf{A} \vec{C}_{L_{j}}+\vec{d}=\vec{P}_{j}+\mathbf{T}_{j} \vec{S}
$$

however, $\vec{C}_{L_{j}}$ cannot be obtained from the force plate, since it is only capable of providing the horizontal $(x-y)$ coordinates of the CoM under static conditions, called $\vec{C}_{W_{j}}$. Accordingly, in the case of static conditions, $\vec{C}_{W_{j}}$ can be obtained as

$$
\left[\begin{array}{ccc}
1 & 0 & 0 \\
0 & 1 & 0
\end{array}\right] \vec{C}_{L_{j}}=\hat{\mathbf{I}} \vec{C}_{L_{j}}=\vec{C}_{W_{j}}=\left\{\begin{array}{c}
c_{w_{x}} \\
c_{w_{y}}
\end{array}\right\}
$$

where $\mathbf{I}$ projects a point onto the $x-y$ plane. Note the assumption that the vertical directions of the vision system and force plate frames are aligned making $\mathbf{A}$ in (3.5) a rotation about $z$. Substituting (3.7) into (3.5) and solving for $\vec{C}_{W_{j}}$ yields

$$
\vec{C}_{W_{j}}=\hat{\mathbf{I}} \mathbf{A}^{\mathbf{T}}\left(\vec{C}_{j}-\vec{d}\right)=\tilde{\mathbf{A}}\left(\vec{C}_{j}-\vec{d}\right)
$$

noting that $\tilde{\mathbf{A}}=\hat{\mathbf{I}} \mathbf{A}^{\mathbf{T}} \in \mathbb{R}^{2 \times 3}$ and

$$
\tilde{\mathbf{A}}^{\mathbf{T}} \tilde{\mathbf{A}}=\left[\begin{array}{lll}
1 & 0 & 0 \\
0 & 1 & 0 \\
0 & 0 & 0
\end{array}\right]=\tilde{\mathbf{I}}
$$

Due to the lack of information about the vertical component of the CoM, the horizontal component of the CoM in the MoCap reference frame may be found from (3.8) by multiplying by $\tilde{\mathbf{A}}^{\mathbf{T}}$,

$$
\tilde{\mathbf{A}}^{\mathbf{T}} \vec{C}_{W_{j}}=\tilde{\mathbf{I}}\left(\vec{C}_{j}-\vec{d}\right)
$$


and observing,

$$
\tilde{\mathbf{I}} \vec{C}_{j}=\tilde{\mathbf{A}}^{\mathbf{T}} \vec{C}_{W_{j}}+\tilde{\mathbf{I}} \vec{d}
$$

The third row of (3.11) is the identity $0=0$. Noting that the identity $\hat{\mathbf{I}} \tilde{\mathbf{I}}=\hat{\mathbf{I}}$, multiplying (3.11) by $\hat{\mathbf{I}}$ yields

$$
\vec{C}_{j}^{*}=\hat{\mathbf{I}} \vec{C}_{j}=\hat{\mathbf{I}} \tilde{\mathbf{A}}^{\mathbf{T}} \vec{C}_{W_{j}}+\hat{\mathbf{I}} \vec{d}=\hat{\mathbf{I}} \mathbf{A} \hat{\mathbf{I}}^{\mathbf{T}} \vec{C}_{W_{j}}+\hat{\mathbf{I}} \vec{d}
$$

where $\vec{C}_{j}^{*} \in \mathbb{R}^{2}$ is the horizontal component of the CoM obtained from the force plate. From (3.4),

$$
\hat{\mathbf{I}} \vec{C}_{j}=\hat{\mathbf{I}}\left(\vec{P}_{j}+\mathbf{T}_{j} \vec{S}\right)=\hat{\mathbf{I}} \vec{P}_{j}+\hat{\mathbf{I}} \mathbf{T}_{j} \vec{S}
$$

and finally,

$$
\vec{C}_{j}^{*}=\vec{P}_{j}^{*}+\mathbf{T}_{j}^{*} \vec{S}
$$

where $\vec{P}_{j}^{*}=\hat{\mathbf{I}} \vec{P}_{j} \in \mathbb{R}^{2}$ and $\mathbf{T}_{j}^{*}=\hat{\mathbf{I}} \mathbf{T}_{j} \in \mathbb{R}^{2 \times 13}$. The terms in (3.14) now relate the data obtained from the force plate, $\vec{C}_{j}^{*}$, to the data obtained from MoCap, $\vec{P}_{j}^{*}$ and $\mathbf{T}_{j}^{*}$.

To find the SESC parameters, gather many instances of (3.14) experimentally such that

$$
\vec{\kappa}=\left\{\begin{array}{c}
\vec{C}_{1}^{*}-\vec{P}_{1}^{*} \\
\vdots \\
\vec{C}_{n}^{*}-\vec{P}_{n}^{*}
\end{array}\right\}=\left[\begin{array}{c}
\mathbf{T}_{1}^{*} \\
\vdots \\
\mathbf{T}_{n}^{*}
\end{array}\right] \vec{S}=\mathbf{B} \vec{S},
$$

where, $\vec{\kappa} \in \mathbb{R}^{2 n \times 1}$ and $\mathbf{B} \in \mathbb{R}^{2 n \times 13}$ are known from the data. Solving with the pseudo-inverse,

$$
\vec{S}=\mathbf{B}^{+} \vec{\kappa}
$$

The $\vec{S}$ contains the parameters which embody the kinematic and static parameters of the entire system. Accordingly, the CoM of the articulated system can now, and without need of a force plate, be obtained from (3.4) including the component in the vertical direction. The result is that the CoM location of the original branched chain is modeled by the terminal point of an appropriately sized spatial serial chain as shown in Fig. 3.3. 
Given the SESC parameters $s_{i}$ in (3.3), observe that a unique set of mass and kinematic parameters for the chain generating the SESC is not feasible. For example, given the values of $s_{1}$ through $s_{13}$, the values in $m_{i}, \lambda_{i}$, and $M$ constitute 27 unknowns in 13 relationships. Even with the availability of $M$ via the force plate, (3.3) still contains 26 unknowns. This notion generalizes to all SESC chains.

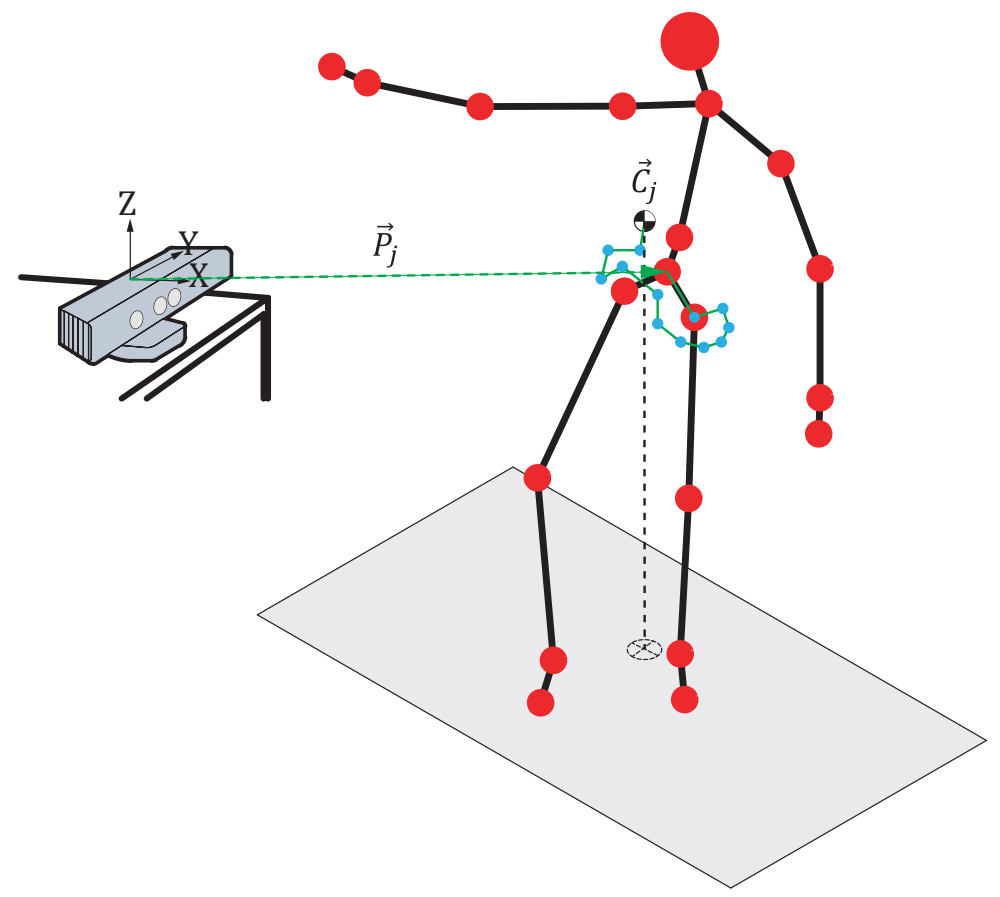

Figure 3.3: The node-based SESC relying solely on the MoCap system to locate the CoM of the human subject is composed of 13 rigid links.

\subsection{Using a Donor Model to Generate the SESC of a New Subject}

The modeling process for estimating the CoM with a SESC requires humanoid or human subjects to perform a potentially significant number of static postures. In the case of humanoid robots, a relatively large number of poses is not an issue. In contrast, for humans, specifically elderly subjects or people with limited locomotion, performing a large number of poses is an issue. Thus, it is 
essential to minimize the number of poses required by people with limited motion. The node-based SESC donor model is a method for using a smaller number of static postures to estimate a new set of SESC parameters given the SESC of a related subject. The new set of SESC parameters may then be used to estimate the CoM of subjects with limited motion.

Given $\mathbf{A}$ and $\vec{d}$ of the force plate relative to the MoCap from the method described in Chapter II, assume that the known SESC parameters of a donor are given by $\vec{S}_{D} \in \mathbb{R}^{13}$. The desired vector $\vec{S}_{N}$ corresponds to the unknown SESC of the new subject. The CoM of the new subject is determined from the MoCap as

$$
\vec{C}_{N}=\vec{P}_{N}+\mathbf{T}_{\mathbf{N}} \vec{S}_{N}
$$

where $\vec{C}_{N} \in \mathbb{R}^{3}$ is the CoM of the new subject, $\vec{P}_{N} \in \mathbb{R}^{3}$ is the position of the hip center relative to the MoCap and $\mathbf{T}_{\mathbf{N}} \in \mathbb{R}^{3 \times 13}$ contains the relative node locations $\vec{\tau}_{1}$ through $\vec{\tau}_{13}$ of the subject skeleton as shown in Fig. 3.2. As both $\vec{S}_{D}$ and $\vec{S}_{N}$ are constant,

$$
\vec{S}_{D}=\vec{S}_{N}+\delta \vec{S}
$$

Observe that if $\delta \vec{S}$ and $\vec{S}_{D}$ are known, then the SESC parameters of the new subject, $\vec{S}_{N}$, can be readily obtained from (3.18). Using the donor SESC parameters and estimating the CoM of the new subject,

$$
\vec{C}_{E}=\vec{P}_{N}+\mathbf{T}_{\mathbf{N}} \vec{S}_{D}
$$

where $\vec{C}_{E} \in \mathbb{R}^{3}$ is the CoM of the new subject using the SESC parameters of the donor as an initial estimate. Substituting (3.18) into (3.19) and expanding yields

$$
\vec{C}_{E}=\vec{P}_{N}+\mathbf{T}_{\mathbf{N}}\left(\vec{S}_{N}+\delta \vec{S}\right)=\vec{P}_{N}+\mathbf{T}_{\mathbf{N}} \vec{S}_{N}+\mathbf{T}_{\mathbf{N}} \delta \vec{S}
$$

Substituting (3.17) into (3.20) yields

$$
\vec{C}_{E}=\vec{C}_{N}+\mathbf{T}_{\mathbf{N}} \delta \vec{S}
$$


Observing that the force plate and MoCap have separate frames of reference,

$$
\vec{C}_{N}=\mathbf{A} \vec{C}_{N_{L}}+\vec{d}
$$

where $\vec{C}_{N_{L}} \in \mathbb{R}^{3}$ is the CoM in the force plate reference frame. As before, $\vec{C}_{N_{L}}$ cannot be obtained from the force plate as it is only capable of providing the horizontal $(x-y)$ coordinates of the CoM under static conditions. This horizontal coordinates are called $\vec{C}_{N_{W}}$. Thus, as in the derivation in Section 3.2,

$$
\vec{C}_{N}^{*}=\hat{\mathbf{I}} \vec{C}_{N}=\hat{\mathbf{I}} \mathbf{A} \hat{\mathbf{I}}^{\mathbf{T}} \vec{C}_{N_{W}}+\hat{\mathbf{I}} \vec{d}
$$

Multiplying (3.21) by $\hat{\mathbf{I}}$,

$$
\vec{C}_{E}^{*}-\vec{C}_{N}^{*}=\mathbf{T}_{\mathbf{N}}^{*} \delta \vec{S}
$$

The value of $\vec{C}_{E}^{*}$ is obtained from the donor's SESC and MoCap data and $\vec{C}_{N}^{*}$ is obtained from the new subject via the force plate. Solve for $\delta \vec{S}$ from (3.24) by appending many instances as

$$
\vec{\varrho}=\left\{\begin{array}{c}
\vec{C}_{E_{1}}^{*}-\vec{C}_{N_{1}}^{*} \\
\vdots \\
\vec{C}_{E_{k}}^{*}-\vec{C}_{N_{k}}^{*}
\end{array}\right\}=\left[\begin{array}{c}
\mathbf{T}_{\mathbf{N} 1}^{*} \\
\vdots \\
\mathbf{T}_{\mathbf{N} k}^{*}
\end{array}\right] \delta \vec{S}=\mathbf{B} \delta \vec{S},
$$

where $k$ is the number of instances. Via the pseudo-inverse,

$$
\delta \vec{S}=\mathbf{B}^{+} \vec{\varrho}
$$

Now the SESC parameters $\vec{S}_{N}$ of the new subject are obtained from (3.18).

\subsection{Constructing a SESC in the Presence of a Static Body}

This model is capable of handling the presence of a static body in the workspace, to provide the needed support for the subjects, without relying on any additional sensors. The position of the CoM $(\vec{C})$, of the $j^{\text {th }}$ posture, for the system of 13 bodies depicted in Fig. 3.4 is

$$
\vec{C}_{j}=M_{s} \vec{P}_{j}+\sum_{i=1}^{13} \vec{\tau}_{i_{j}} s_{i}^{*}+M_{o} \vec{P}_{o}
$$


where, the SESC parameters $s_{1}^{*}$ through $s_{13}^{*}$ are:

$$
\begin{aligned}
& s_{1}^{*}=\frac{m_{1} \lambda_{1}+m_{2}+m_{3}}{M_{t}}, \quad s_{2}^{*}=\frac{m_{2} \lambda_{2}+m_{3}}{M_{t}}, \quad s_{3}^{*}=\frac{m_{3} \lambda_{3}}{M_{t}}, \quad s_{4}^{*}=\frac{m_{4} \lambda_{4}+m_{5}+m_{6}}{M_{t}}, \\
& s_{5}^{*}=\frac{m_{5} \lambda_{5}+m_{6}}{M_{t}}, \quad s_{6}^{*}=\frac{m_{6} \lambda_{6}}{M_{t}}, \quad s_{7}^{*}=\frac{m_{7} \lambda_{7}+m_{8}+m_{9}+m_{10}+m_{11}+m_{12}+m_{13}}{M_{t}}, \\
& s_{8}^{*}=\frac{m_{8} \lambda_{8}+m_{9}+m_{10}}{M_{t}}, \quad s_{9}^{*}=\frac{m_{9} \lambda_{9}+m_{10}}{M_{t}}, \quad s_{10}^{*}=\frac{m_{10} \lambda_{10}}{M_{t}}, \\
& s_{11}^{*}=\frac{m_{11} \lambda_{11}+m_{12}+m_{13}}{M_{t}}, \quad s_{12}^{*}=\frac{m_{12} \lambda_{12}+m_{13}}{M_{t}}, \quad s_{13}^{*}=\frac{m_{13} \lambda_{13}}{M_{t}},
\end{aligned}
$$

and

$$
M_{t}=\sum_{i=1}^{13} m_{i}+m_{o}=m_{s}+m_{o}, \quad M_{o}=\frac{m_{o}}{M_{t}}, \quad M_{s}=\frac{m_{s}}{M_{t}} .
$$

Observe that (3.28) is similar to (3.3) but, in this case, contains the kinematic and static parameters of the subject and static body. Rearranging (3.27),

$$
\begin{aligned}
\vec{C}_{j} & =M_{s} \vec{P}_{j}+\left[\begin{array}{llll}
\vec{\tau}_{1_{n}} & \vec{\tau}_{2_{n}} & \ldots & \vec{\tau}_{13_{n}}
\end{array}\right]\left\{\begin{array}{c}
s_{1}^{*} \\
s_{2}^{*} \\
\vdots \\
s_{13}^{*}
\end{array}\right\}+M_{o} \vec{P}_{o} \\
& =M_{s} \vec{P}_{j}+\mathbf{T}_{j} \vec{S}^{*}+M_{o} \vec{P}_{o},
\end{aligned}
$$

where $\vec{S}^{*} \in \mathbb{R}^{13}$ contains the SESC parameters. Equating (3.30) and (3.5) and solving for $\vec{C}_{L_{j}}$,

$$
\vec{C}_{L_{j}}=\mathbf{A}^{\mathbf{T}}\left(M_{s} \vec{P}_{j}+\mathbf{T}_{j} \vec{S}^{*}+M_{o} \vec{P}_{o}-\vec{d}\right)
$$

However, from (3.7) under the static condition $\vec{C}_{W_{j}}$ may be obtained as

$$
\vec{C}_{W_{j}}=\hat{\mathbf{I}} \mathbf{A}^{\mathbf{T}}\left(M_{s} \vec{P}_{j}+\mathbf{T}_{j} \vec{S}^{*}+M_{o} \vec{P}_{o}-\vec{d}\right) .
$$

Using (3.32) with two experimental postures from the MoCap and force plate,

$$
\begin{aligned}
& \vec{C}_{W_{j}}=\tilde{\mathbf{A}}\left(M_{s} \vec{P}_{j}+\mathbf{T}_{j} \vec{S}^{*}+M_{o} \vec{P}_{o}-\vec{d}\right), \\
& \vec{C}_{W_{j+1}}=\tilde{\mathbf{A}}\left(M_{s} \vec{P}_{j+1}+\mathbf{T}_{j+1} \vec{S}^{*}+M_{o} \vec{P}_{o}-\vec{d}\right) .
\end{aligned}
$$


Subtracting the two instances in (3.33) yields

$$
\Delta \vec{C}=\tilde{\mathbf{A}}\left(M_{s} \Delta \vec{P}+\Delta \mathbf{T} \vec{S}^{*}\right)=\left\{\begin{array}{l}
\delta c_{x} \\
\delta c_{y}
\end{array}\right\}
$$

noting that the term corresponding to the static body is eliminated as a result of this difference. To find the SESC parameters, solve for $\vec{S}^{*}$ from (3.34) by appending many instances as

$$
\vec{\kappa}=\left\{\begin{array}{c}
\Delta \vec{C}_{1}-\tilde{\mathbf{A}} M_{s} \Delta \vec{P}_{1} \\
\vdots \\
\Delta \vec{C}_{k}-\tilde{\mathbf{A}} M_{s} \Delta \vec{P}_{k}
\end{array}\right\}=\left[\begin{array}{c}
\tilde{\mathbf{A}} \Delta \mathbf{T}_{1} \\
\vdots \\
\tilde{\mathbf{A}} \Delta \mathbf{T}_{k}
\end{array}\right] \vec{S}^{*}=\mathbf{B} \vec{S}^{*}
$$

where, $\vec{\kappa} \in \mathbb{R}^{2 k \times 1}, \mathbf{B} \in \mathbb{R}^{2 k \times 13}$, and

$$
\vec{S}^{*}=\mathbf{B}^{+} \vec{\kappa}
$$

The $\vec{S}^{*}$ contains the kinematic and static parameters of the entire system including the static body. The $\vec{S}$ that describes only the subject can be determined as

$$
\vec{S}=\frac{M_{t}}{m_{s}} \vec{S}^{*}
$$

Note that $\vec{S}$ contains the links properties of the SESC chain for the subject. The CoM of the subject for a given instant can be obtained from (3.30) by setting $m_{o}=0$, which results in the same expression as (3.4) from Section 3.2 for the CoM of the subject. Hence, $\vec{C}_{j}$ is readily obtained without the need of a force plate. Also observe that the addition of the static body to the modeling process is handled without the need for any additional equipment. The introduction of a static body such as a wheelchair or walker will improve the balance of many subjects and will allow for more diverse postures.

\subsection{Arbitrary Location of the CoM Associated with the Torso}

A further extension to node-based SESC modeling is allowing an arbitrary location of the CoM associated with the torso via the introduction of a relevant moving frame. Note that this process can be done for any body for which the assumption that the CoM lies along the vector between nodes 


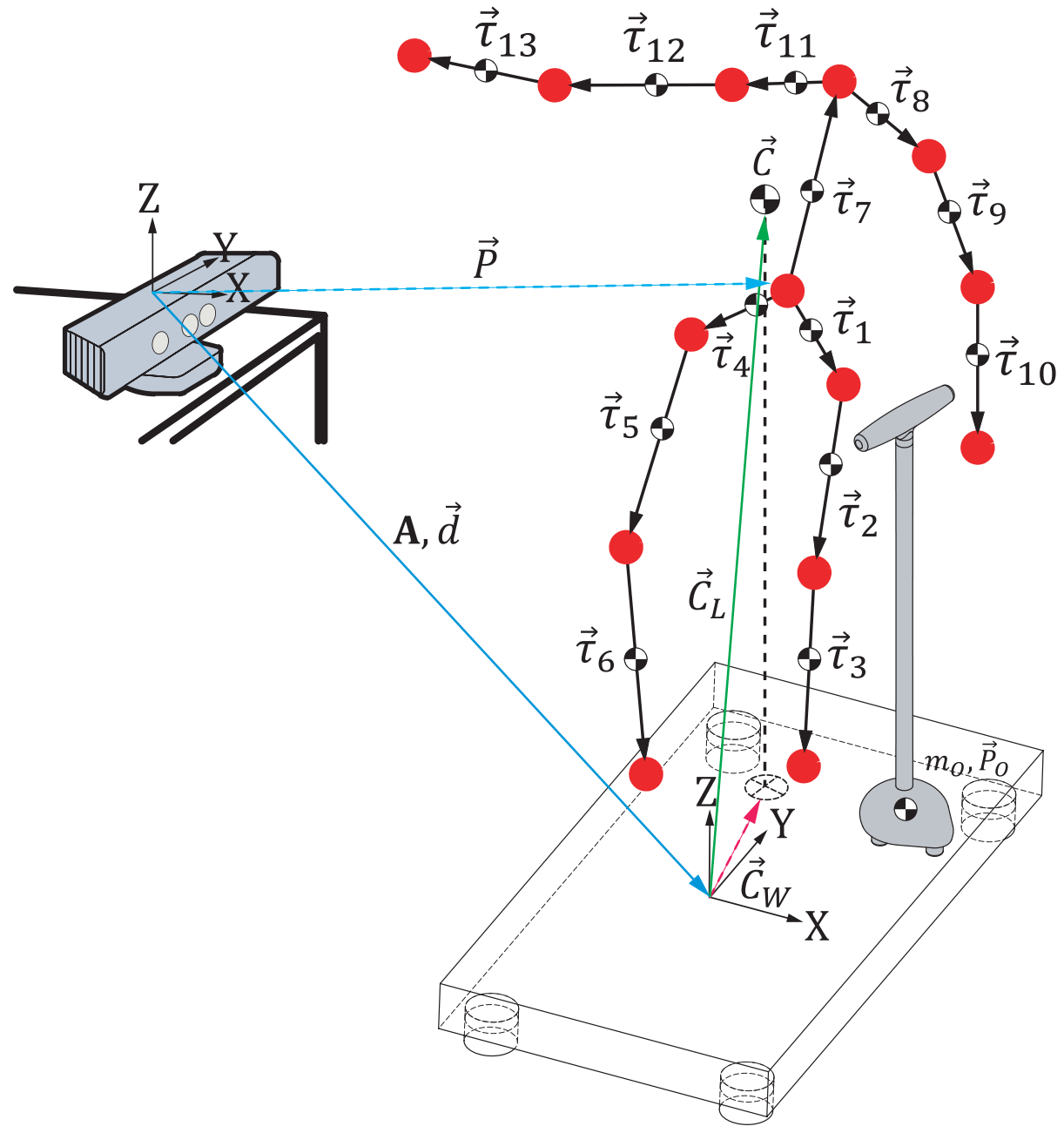

Figure 3.4: The skeleton model of a human subject composed of 13 links including the static body to provide the needed balance/stability for subjects or while preforming unconventional body postures. 
may be questioned. The torso is the focus of this section due to that being the body part readily questioned in the modeling of a humanoid or human from nodes.

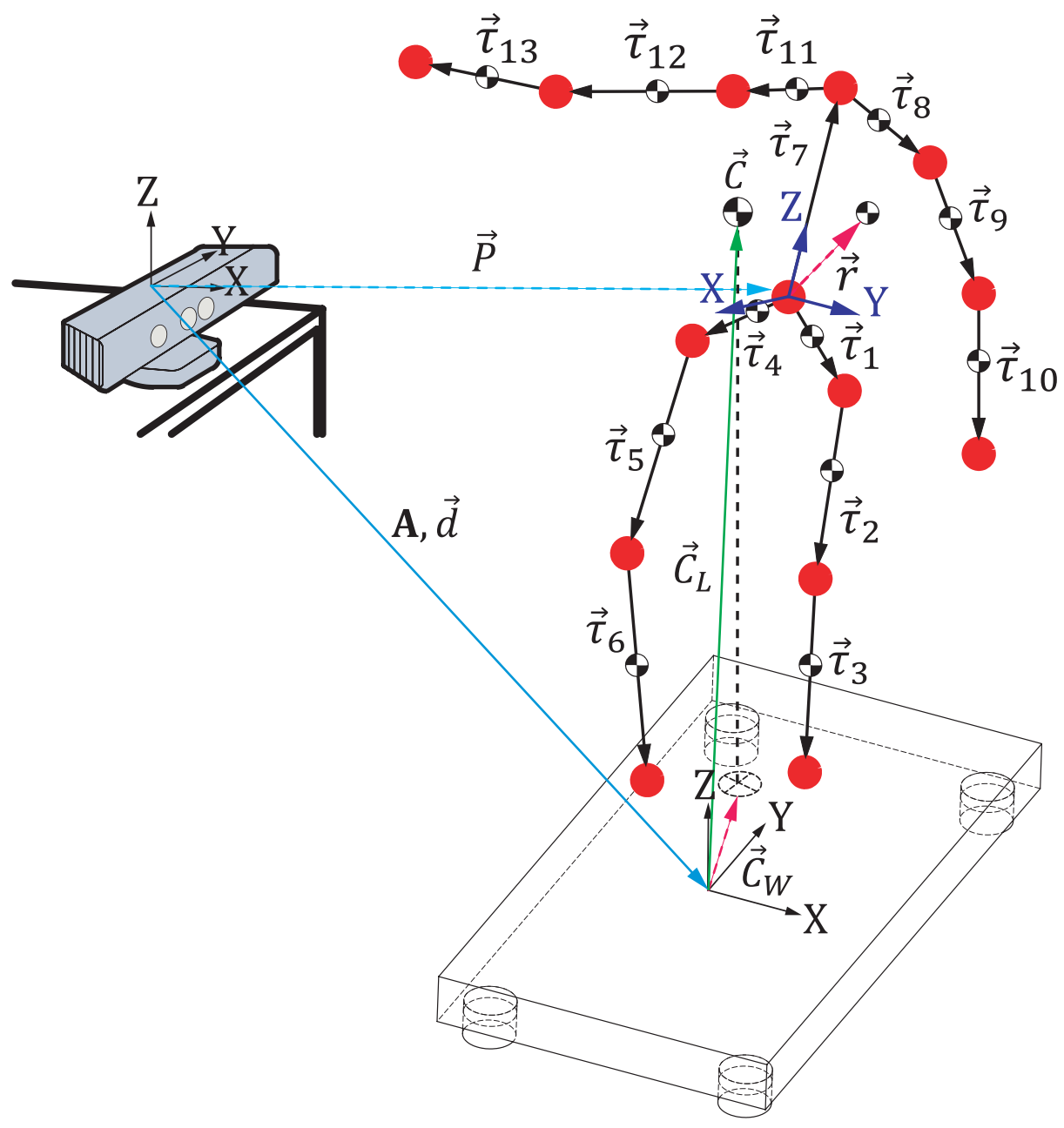

Figure 3.5: The skeleton model of a human subject composed of 13 links. The kinematic and static parameters of the subject are show as well as the moving frame attached to the torso.

The position of the CoM $(\vec{C})$, of the $j^{\text {th }}$ posture, for the system of 13 bodies depicted in Fig. 3.5 is

$$
\vec{C}_{j}=\vec{P}_{j}+\sum_{i=1}^{13} \vec{\tau}_{i_{j}} s_{i}+\mathbf{R}_{j} \vec{s}_{t}
$$


where, $\vec{s}_{t}=\frac{m_{7} \vec{r}}{M}$ and $\vec{r} \in \mathbb{R}^{3}$ is the CoM of the torso in the local reference frame attached to the torso. Note that the SESC parameters $s_{1}-s_{6}$ and $s_{8}-s_{13}$ are identical to (3.3), whereas $s_{7}=$ $\frac{m_{8}+m_{9}+m_{10}+m_{11}+m_{12}+m_{13}}{M}$. The matrix $\mathbf{R}_{j} \in \mathrm{SO}(3)$ is the rotation matrix of the spine joint and may be modeled as the product of three rotation matrices,

$$
\mathbf{R}_{j}=\mathbf{A}_{y}(\theta) \mathbf{A}_{z}(\phi) \mathbf{A}_{x}(\psi)
$$

where the subscripts $x, y$, and $z$ represent the axis of rotation and the $\theta, \phi$, and $\psi$ are the angles of rotation relative to the MoCap. The reference frame is attached in accordance with the procedure described by Kinect [63] wherein the rotation angles in (3.39) can be obtained from the Kinect output data. Rearranging (3.38),

$$
\begin{aligned}
\vec{C}_{j}=\vec{P}_{j}+\left[\begin{array}{llllll}
\vec{\tau}_{1_{j}} & \vec{\tau}_{2_{j}} & \ldots & \mathbf{R}_{j} & \ldots & \vec{\tau}_{13_{j}}
\end{array}\right]\left\{\begin{array}{c}
s_{1} \\
s_{2} \\
\vdots \\
\vec{s}_{t} \\
\vdots \\
s_{13}
\end{array}\right\} \\
=\vec{P}_{j}+\mathbf{T}_{j} \vec{S},
\end{aligned}
$$

where $\vec{S} \in \mathbb{R}^{16}$ contains the SESC parameters and $\mathbf{T}_{j} \in \mathbb{R}^{3 \times 16}$ contains the relative node locations $\vec{\tau}_{1_{j}}$ through $\vec{\tau}_{13_{j}}$ and $\mathbf{R}_{j}$. Note that the introduction of a moving frame adds three more SESC parameters, $s_{t_{1}}$ through $s_{t_{3}}$ and $\mathbf{R}_{j}$ to the modeling. Observe that the process of finding the CoM is identical to Section 3.2.

\subsection{Model Construction of a Human Subject where A and $\vec{d}$ are Unknown}

Section 3.2 determined the node-based SESC model when the relative location of the force plate was known. This section introduces an alternative way to estimate $\mathbf{A}$ and $\vec{d}$ from the MoCap and force plate data itself. For the same system of 13 bodies depicted in Fig. 3.2, (3.1) through (3.8) are 
identical. From (3.6) solve for $\vec{C}_{L_{j}}$,

$$
\vec{C}_{L_{j}}=\mathbf{A}^{\mathbf{T}}\left(\vec{P}_{j}+\mathbf{T}_{j} \vec{S}-\vec{d}\right)
$$

However, from (3.7) under the static condition $\vec{C}_{W_{j}}$ may be obtained as

$$
\vec{C}_{W_{j}}=\hat{\mathbf{I}} \mathbf{A}^{\mathbf{T}}\left(\vec{P}_{j}+\mathbf{T}_{j} \vec{S}-\vec{d}\right)
$$

Using (3.42) with two experimental postures from the MoCap and force plate,

$$
\begin{aligned}
& \vec{C}_{W_{j}}=\tilde{\mathbf{A}}\left(\vec{P}_{j}+\mathbf{T}_{j} \vec{S}-\vec{d}\right), \\
& \vec{C}_{W_{j+1}}=\tilde{\mathbf{A}}\left(\vec{P}_{j+1}+\mathbf{T}_{j+1} \vec{S}-\vec{d}\right) .
\end{aligned}
$$

Subtracting the two instances in (3.43) yields

$$
\Delta \vec{C}=\tilde{\mathbf{A}}(\Delta \vec{P}+\Delta \mathbf{T} \vec{S})=\left\{\begin{array}{l}
\delta c_{x} \\
\delta c_{y}
\end{array}\right\}
$$

noting that the term corresponding to the displacement $(\vec{d})$ is eliminated as a result of the subtraction process. Equation (3.44) for the $k^{\text {th }}$ experimental posture may be partitioned as

$$
\begin{aligned}
\delta c_{x_{k}} & =\left[\begin{array}{lll}
x_{1} & y_{1} & z_{1}
\end{array}\right]\left(\Delta \vec{P}_{k}+\Delta \mathbf{T}_{k} \vec{S}\right), \\
\delta c_{y_{k}} & =\left[\begin{array}{lll}
x_{2} & y_{2} & z_{2}
\end{array}\right]\left(\Delta \vec{P}_{k}+\Delta \mathbf{T}_{k} \vec{S}\right) .
\end{aligned}
$$

Expanding (3.45) for $\delta c_{x_{k}}$ and $\delta c_{y_{k}}$,

$$
\begin{aligned}
& \delta c_{x_{k}}=x_{1} \delta p_{x_{k}}+y_{1} \delta p_{y_{k}}+z_{1} \delta p_{z_{k}} \\
& +\sum_{i=1}^{13} \delta \tau_{[1, i]_{k}} s_{i} x_{1}+\sum_{i=1}^{13} \delta \tau_{[2, i]_{k}} s_{i} y_{1}+\sum_{i=1}^{13} \delta \tau_{[3, i]_{k}} s_{i} z_{1} \\
& \delta c_{y_{k}}=x_{2} \delta p_{x_{k}}+y_{2} \delta p_{y_{k}}+z_{2} \delta p_{z_{k}} \\
& +\sum_{i=1}^{13} \delta \tau_{[1, i]_{k}} s_{i} x_{2}+\sum_{i=1}^{13} \delta \tau_{[2, i]_{k}} s_{i} y_{2}+\sum_{i=1}^{13} \delta \tau_{[3, i]_{k}} s_{i} z_{2} .
\end{aligned}
$$


Rearranging (3.46) and acquiring enough instances yields

$$
\begin{aligned}
& \left\{\begin{array}{c}
\delta c_{x_{1}} \\
\delta c_{x_{2}} \\
\vdots \\
\delta c_{x_{k}}
\end{array}\right\}=\left[\begin{array}{cccccc}
\delta p_{x_{1}} & \ldots & \delta p_{z_{1}} & \delta \tau_{[1,1]_{1}} & \ldots & \delta \tau_{[3,13]_{1}} \\
\delta p_{x_{2}} & \ldots & \delta p_{z_{2}} & \delta \tau_{[1,1]_{2}} & \ldots & \delta \tau_{[3,13]_{2}} \\
\vdots & \vdots & \vdots & \ldots & \ddots & \vdots \\
\delta p_{x_{k}} & \ldots & \delta p_{z_{k}} & \delta \tau_{[1,1]_{k}} & \ldots & \delta \tau_{[3,13]_{k}}
\end{array}\right]\left\{\begin{array}{c}
x_{1} \\
y_{1} \\
z_{1} \\
s_{1} x_{1} \\
s_{1} y_{1} \\
s_{1} z_{1} \\
\vdots \\
s_{13} x_{1} \\
s_{13} y_{1} \\
s_{13} z_{1}
\end{array}\right\} \\
& \left\{\begin{array}{c}
\delta c_{y_{1}} \\
\delta c_{y_{2}} \\
\vdots \\
\delta c_{y_{k}}
\end{array}\right\}=\left[\begin{array}{cccccc}
\delta p_{x_{1}} & \ldots & \delta p_{z_{1}} & \delta \tau_{[1,1]_{1}} & \ldots & \delta \tau_{[3,13]_{1}} \\
\delta p_{x_{2}} & \ldots & \delta p_{z_{2}} & \delta \tau_{[1,1]_{2}} & \ldots & \delta \tau_{[3,13]_{2}} \\
\vdots & \vdots & \vdots & \ldots & \ddots & \vdots \\
\delta p_{x_{k}} & \ldots & \delta p_{z_{k}} & \delta \tau_{[1,1]_{k}} & \ldots & \delta \tau_{[3,13]_{k}}
\end{array}\right]\left\{\begin{array}{c}
x_{2} \\
y_{2} \\
z_{2} \\
s_{1} x_{2} \\
s_{1} y_{2} \\
s_{1} z_{2} \\
\vdots \\
s_{13} x_{2} \\
s_{13} y_{2} \\
s_{13} z_{2}
\end{array}\right\}
\end{aligned}
$$

Equation (3.47) is represented as

$$
\Delta \vec{C}_{x}=\mathbf{D} \vec{\varepsilon}_{1}, \quad \Delta \vec{C}_{y}=\mathbf{D} \vec{\varepsilon}_{2},
$$

where $\Delta \vec{C}_{x}$ and $\Delta \vec{C}_{y} \in \mathbb{R}^{k \times 1}, \mathbf{D} \in \mathbb{R}^{k \times 42}, \vec{\varepsilon}_{1}$ and $\vec{\varepsilon}_{2} \in \mathbb{R}^{42 \times 1}$. Via the pseudo-inverse,

$$
\vec{\varepsilon}_{1}=\mathbf{D}^{+} \Delta \vec{C}_{x}, \quad \vec{\varepsilon}_{2}=\mathbf{D}^{+} \Delta \vec{C}_{y}
$$

Extract from the vector $\vec{\varepsilon}_{1}$ in (3.49) the values for $x_{1}, y_{1}$ and $z_{1}$, and from the vector $\vec{\varepsilon}_{2}$ the values for $x_{2}, y_{2}$ and $z_{2}$. These values are normalized to produce the unit vectors of $\tilde{\mathbf{A}}$ as

$$
\vec{X}=\frac{\left\{\begin{array}{lll}
x_{1} & y_{1} & z_{1}
\end{array}\right\}^{T}}{\left\|\left\{\begin{array}{lll}
x_{1} & y_{1} & z_{1}
\end{array}\right\}\right\|}, \quad \vec{Y}=\frac{\left\{\begin{array}{lll}
x_{2} & y_{2} & z_{2}
\end{array}\right\}^{T}}{\left\|\left\{\begin{array}{lll}
x_{2} & y_{2} & z_{2}
\end{array}\right\}\right\|}, \quad \tilde{\mathbf{A}}=\left[\begin{array}{c}
\vec{X}^{T} \\
\vec{Y}^{T}
\end{array}\right] .
$$

From $\vec{Z}=\vec{X} \times \vec{Y}$, the three vectors combine to form a numerical approximation of $\mathbf{A}$ as

$$
\mathbf{A}=\left[\begin{array}{lll}
\vec{X} & \vec{Y} & \vec{Z}
\end{array}\right]
$$


To find the SESC parameters, solve for $\vec{S}$ from (3.44) by appending many instances as

$$
\vec{\kappa}=\left\{\begin{array}{c}
\Delta \vec{C}_{1}-\tilde{\mathbf{A}} \Delta \vec{P}_{1} \\
\vdots \\
\Delta \vec{C}_{k}-\tilde{\mathbf{A}} \Delta \vec{P}_{k}
\end{array}\right\}=\left[\begin{array}{c}
\tilde{\mathbf{A}} \Delta \mathbf{T}_{1} \\
\vdots \\
\tilde{\mathbf{A}} \Delta \mathbf{T}_{k}
\end{array}\right] \vec{S}=\mathbf{B} \vec{S}
$$

where, $\vec{\kappa} \in \mathbb{R}^{2 k \times 1}, \mathbf{B} \in \mathbb{R}^{2 k \times 13}$, and

$$
\vec{S}=\mathbf{B}^{+} \vec{\kappa}
$$

The $\vec{S}$ contains the links properties of the SESC chain. Accordingly, the CoM can be readily obtained from (3.2). Note that the displacement vector $\vec{d}$ is not required by this approach, but if desired the horizontal component can be found from (3.42) by multiplying by $\tilde{\mathbf{A}}^{\mathbf{T}}$,

$$
\tilde{\mathbf{I}} \vec{d}=\tilde{\mathbf{I}} \vec{P}_{j}+\tilde{\mathbf{I}} \mathbf{T}_{j} \vec{S}-\tilde{\mathbf{A}}^{\mathbf{T}} \vec{C}_{W_{j}}
$$

note that, due to $\tilde{\mathbf{I}}$, the third row of (3.54) is identically 0 . Therefore, the none zero rows can be obtained from (3.54) by multiplying by $\hat{\mathbf{I}}$,

$$
\begin{aligned}
\hat{\mathbf{I}} \tilde{\mathbf{I}} \vec{d} & =\hat{\mathbf{I}} \tilde{\mathbf{I}} \vec{P}_{j}+\hat{\mathbf{I}} \tilde{\mathbf{I}} \mathbf{T}_{j} \vec{S}-\hat{\mathbf{I}} \tilde{\mathbf{A}}^{\mathbf{T}} \vec{C}_{W_{j}}, \\
\vec{d}_{j}^{*} & =\vec{P}_{j}^{*}+\mathbf{T}_{j}^{*} \vec{S}-\hat{\mathbf{I}} \tilde{\mathbf{A}}^{\mathbf{T}} \vec{C}_{W_{j}},
\end{aligned}
$$

and the projection of the displacement vector $\vec{d}$, called $\vec{d}_{p}$, is equal to

$$
\overrightarrow{d_{p}}=\frac{1}{n} \sum_{j=1}^{n} \vec{d}_{j}^{*}
$$

Equation (3.55) is observed to calculate only the horizontal component of $\vec{d}$. In fact, due to the projection used in (3.7) because of the force plate reading, the vertical component cannot be estimated from the data. 


\section{CHAPTER IV}

\section{EXPERIMENTAL RESULTS}

The presented methods were validated by conducting several experiments and divided into two categories. The equipment used for data acquisition in the experimental portion of this work includes the Kinect/WBB and VICON/AMTI-OR6 sensors. The first category relies on the Kinect/WBB sensors to verify the node-based SESC method when A and $\vec{d}$ are known, and to validate the donor model. The second category relies on the VICON/AMTI-OR6 sensors and includes an extra body in the workspace to validate the node-based SESC method when $\mathbf{A}$ and $\vec{d}$ are unknown. The Kinect/WBB are used to verify the presented methodology due to their portability, cost, accessibility and simplicity. The VICON/AMTI-OR6 are used for cross validation. This section will cover the relevant details of the two systems.

\subsection{Experimental Protocol}

Microsoft Visual Studio tools were used to develop a Microsoft Windows Foundation (WPF) application capable of processing both the data coming from the WBB and the Kinect sensors simultaneously. During the SESC's experimental construction, the Kinect sensor was placed on a flat stable surface and in such a way as to capture the frontal plane of the subject.

Observing the Kinect/WBB (or the VICON/AMTI-OR6) data simultaneously, both readings need to satisfy the threshold, hence to be nearly static, to attain an accurate estimate for the CoM 


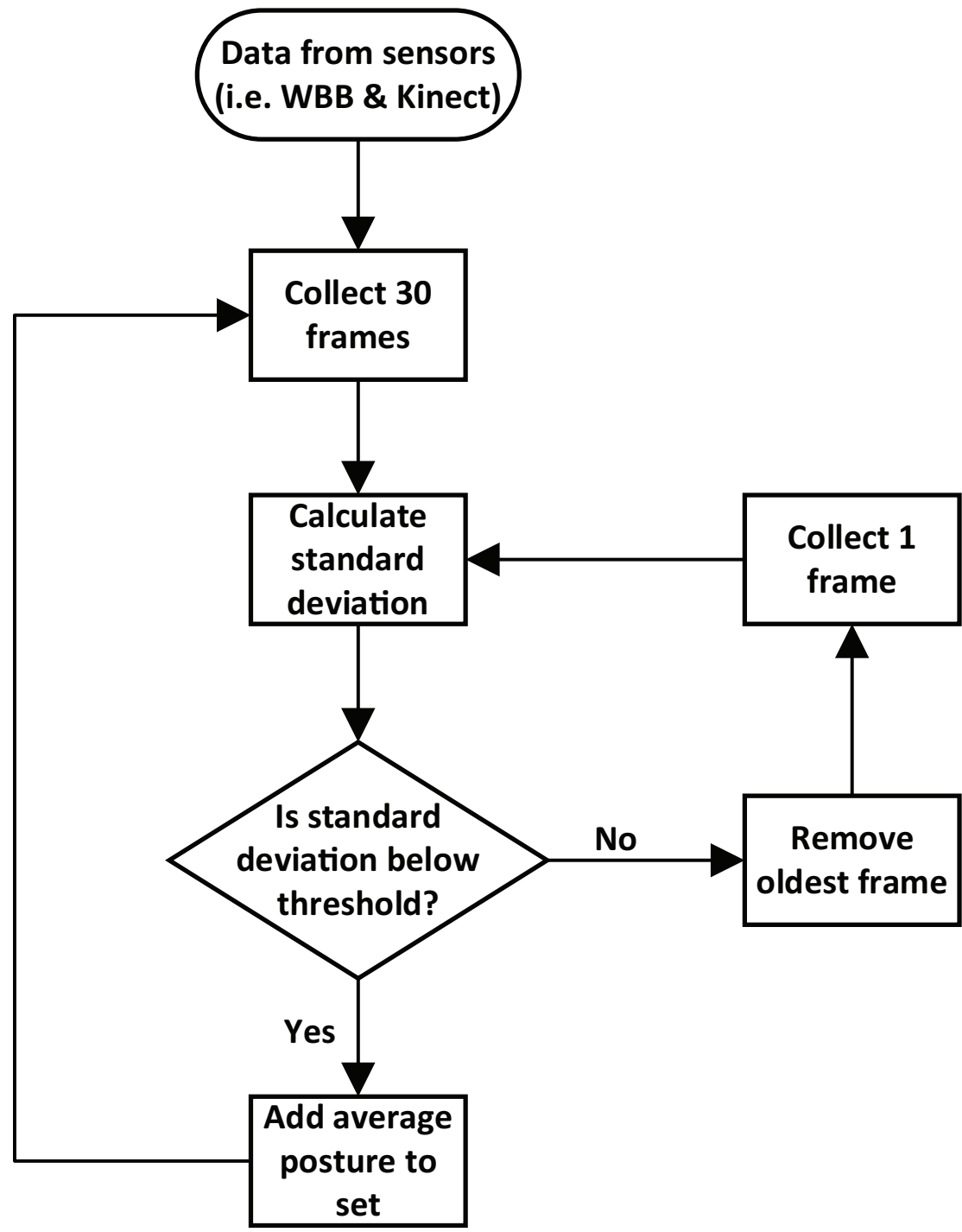

Figure 4.1: The flow chart detailing the process of determining the near static postures. 
projection. To identify if a posture is stable, the CoP position and the subject's nodes are monitored for 1 second, corresponding to 30 frames. Then, for the 30 frames the standard deviation for each of the $x, y$, and $z$ elements of the nodes along with the $x$ and $y$ elements of the CoP are calculated. The posture is considered static if the standard deviation of all of the nodes and CoP locations are below a threshold value for the 1 second window. For the purpose of this test, the standard deviation for the nodes must be $\leq 10 \mathrm{~mm}$, while for the CoP it must be $\leq 3 \mathrm{~mm}$. The subject is notified once a posture is deemed static, the average values are added to the data set and the process starts over. In contrast, if the screening test fails, the algorithm removes the oldest frame and acquires a new one. Then the algorithm evaluates the standard deviations for the new set of frames ( 1 new and 29 old) and repeats the comparison against the threshold. This process continues until a suitable number of postures is acquired as depicted in Fig. 4.1.

For the VICON/AMTI-OR6 case, the CoP position and the subject's nodes are monitored for 6 seconds during the identification phase. The acquired poses are not processed for being near static at the time of data collection. The data are searched in an offline process similar to the one described for Kinect/WBB case. This process generates the required number of poses.

\subsection{Method Validation Where A and $\vec{d}$ are Known}

Chapter II introduced the method of using calibration to estimate the relative orientation (A) and the displacement $(\vec{d})$ between any MoCap system and force plate. Thus, the $\mathbf{A}$ and $\vec{d}$ for the Kinect and the WBB can be readily obtained by this method. The knowledge of $\mathbf{A}$ and $\vec{d}$ simplifies the process and drastically reduces the number of postures required by the subject.

The experimental validation involved three male subjects and one female subject. The female subject (Subject 1) has a height of 1.59 meters and a weight of $53 \mathrm{~kg}$. For the male subjects, the first male subject (Subject 2) has a height of 1.72 meters and weighs $76.6 \mathrm{~kg}$. The second male 
(Subject 3) is 1.8 meters tall and weighs $86 \mathrm{~kg}$. The third male (Subject 4, 5 and 6) is 1.79 meters tall and weighs $69 \mathrm{~kg}$. The subjects are instructed to wear tight non reflective clothing similar to the one seen in Fig. 4.13. Relevant details of the experimental results for the node-based SESC and the donor model are now introduced.

\subsubsection{Method Validation using Kinect/WBB Sensors}

The first part of the three experiments described in this section involves calibrating the Kinect and the WBB. This is done by estimating the $\mathbf{A}$ and $\vec{d}$ by following the procedure described in Chapter II. In short, the procedure uses a target weight placed on top of the WBB and tracked by the Kinect. The target weight is moved to different locations on the WBB workspace and the CoP and position are recorded and used to estimate the required parameters.

\section{Experiment A}

In Experiment A, Subject 1 was instructed to perform a total of 107 static postures avoiding those where the body is turned away from the Kinect and avoiding self-occlusion between body parts. The postures were recorded in approximately 15 minutes. From this data, 13 postures were randomly chosen as the training set. Section 3.6 shows that, theoretically, 13 postures are the minimum required number where the system becomes solvable. The training set was used to identify the SESC parameters for the articulated chain described in Section 3.2. As a result, the position of the CoM of the original branched chain is modeled by the terminal point of a virtual spatial serial chain of appropriate size. The serial chain is depicted by the green dashed lines in Fig. 4.2 where the terminal point terminates at the CoM.

The remaining 94 postures were used as the test set to evaluate the CoM. The predicted CoM is then compared to the measured CoM obtained from the force plate as depicted in Tab. 4.1 and referred to as "Distance". The CoM in the plane of the force plate is seen to have an average distance 


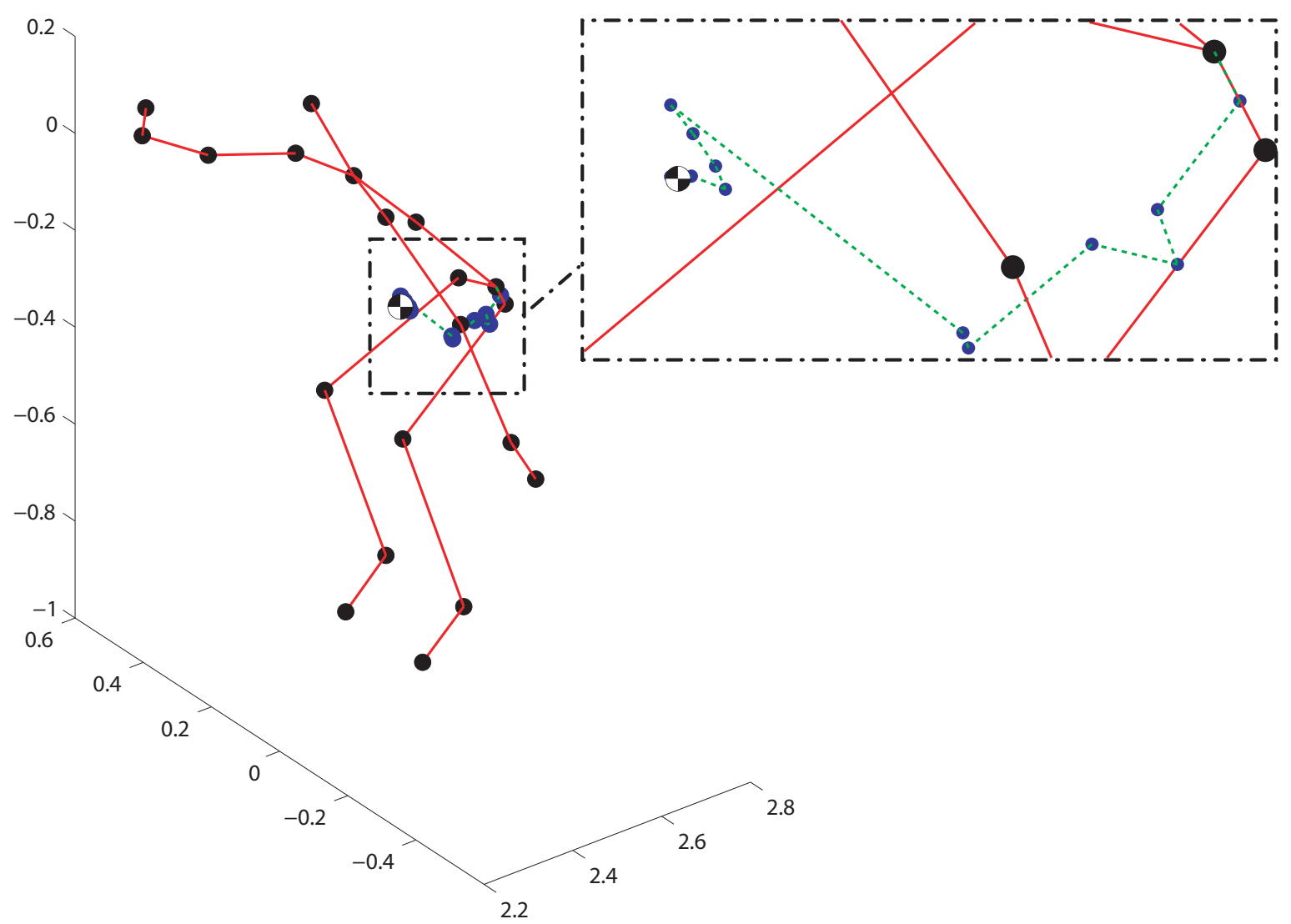

Figure 4.2: The SESC model of Subject 1 where the SESC, depicted by the green dashed lines, terminates at the CoM. 
of $12.64 \mathrm{~mm}$ and a root mean square error (RMSE) of $10.53 \mathrm{~mm}$ in the $x$ direction and $9.72 \mathrm{~mm}$ in the $y$ direction. The worst $\Delta x$ is $35.07 \mathrm{~mm}$ and the worst $\Delta y$ is $-23.26 \mathrm{~mm}$.

To observe the effect of the number of postures to the method accuracy, the 107 postures were divided into two sets. The first set included 77 postures as the training set to evaluate the SESC parameters of Subject 1. The second set was composed of the remaining 30 postures and was used as the testing set to evaluate the CoM. The SESC parameters were evaluated starting from 13 postures, in single posture increments. The average distance is plotted against the number of postures and the results are shown in Fig. 4.3. Observe that as the number of postures increases the error in the average distance decreases and reaches a point where the error remains relatively constant. Note that the average error is $8.28 \mathrm{~mm}$ when using all of the 77 postures.

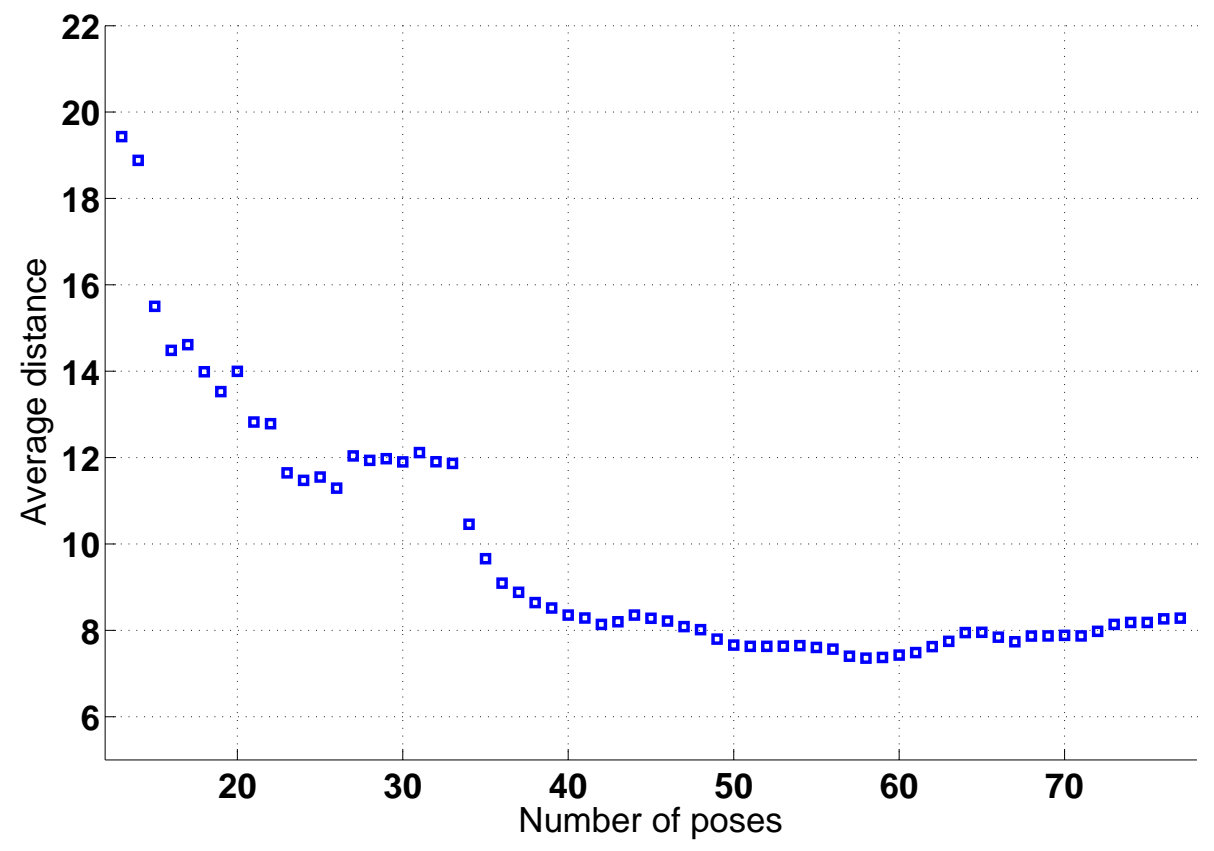

Figure 4.3: The average distance of the CoM for Subject 1 for the testing set of 30 postures as a function of the number of experimental postures. 
Table 4.1: The CoM positions for Subject 1 as predicted by the node-based SESC method for the 94 postures using the data from the Kinect/WBB sensors. The table includes the deviations of the predicted CoM from the measured CoM for postures 1 through 47 .

\begin{tabular}{|c|c|c|c|c|c|c|c|}
\hline \multirow[b]{2}{*}{ Postures } & \multicolumn{2}{|c|}{ Predicted CoM (mm) } & \multicolumn{2}{|c|}{ Measured CoM $(\mathrm{mm})$} & \multicolumn{2}{|c|}{ Error $(\mathrm{mm})$} & \multirow{2}{*}{$\frac{\text { Distance }(\mathrm{mm})}{d}$} \\
\hline & $x$ & $y$ & $x$ & $y$ & $\Delta x$ & $\Delta y$ & \\
\hline 1 & 91.80 & 2512.97 & 81.77 & 2527.60 & 10.03 & -14.63 & 17.73 \\
\hline 2 & 170.51 & 2489.06 & 178.49 & 2504.72 & -7.99 & -15.66 & 17.58 \\
\hline 3 & 57.71 & 2498.72 & 59.24 & 2483.23 & -1.53 & 15.49 & 15.56 \\
\hline 4 & -78.69 & 2528.24 & -91.27 & 2532.74 & 12.58 & -4.50 & 13.36 \\
\hline 5 & 67.29 & 2525.50 & 53.55 & 2514.80 & 13.74 & 10.71 & 17.42 \\
\hline 6 & 51.07 & 2534.39 & 52.73 & 2532.45 & -1.66 & 1.94 & 2.55 \\
\hline 7 & 190.49 & 2483.81 & 193.74 & 2499.92 & -3.25 & -16.11 & 16.43 \\
\hline 8 & 182.88 & 2501.91 & 183.88 & 2486.55 & -1.00 & 15.37 & 15.40 \\
\hline 9 & 52.36 & 2522.94 & 49.76 & 2523.61 & 2.60 & -0.67 & 2.68 \\
\hline 10 & 58.63 & 2520.18 & 54.57 & 2527.32 & 4.05 & -7.14 & 8.21 \\
\hline 11 & -76.84 & 2504.60 & -98.79 & 2520.64 & 21.96 & -16.04 & 27.19 \\
\hline 12 & 56.85 & 2552.36 & 57.19 & 2543.48 & -0.34 & 8.88 & 8.88 \\
\hline 13 & 190.58 & 2513.73 & 197.76 & 2527.38 & -7.18 & -13.65 & 15.42 \\
\hline 14 & -90.80 & 2518.66 & -87.68 & 2523.73 & -3.12 & -5.07 & 5.96 \\
\hline 15 & 192.86 & 2504.61 & 204.85 & 2497.09 & -11.99 & 7.53 & 14.15 \\
\hline 16 & -59.65 & 2515.46 & -72.99 & 2531.10 & 13.33 & -15.64 & 20.55 \\
\hline 17 & 56.88 & 2532.02 & 52.50 & 2527.48 & 4.38 & 4.54 & 6.31 \\
\hline 18 & 58.47 & 2536.55 & 51.91 & 2538.22 & 6.56 & -1.68 & 6.77 \\
\hline 19 & 85.63 & 2559.22 & 74.16 & 2566.46 & 11.48 & -7.24 & 13.57 \\
\hline 20 & 63.77 & 2530.46 & 56.26 & 2536.01 & 7.51 & -5.55 & 9.34 \\
\hline 21 & -73.84 & 2500.10 & -90.19 & 2513.44 & 16.35 & -13.35 & 21.11 \\
\hline 22 & 190.97 & 2545.30 & 202.84 & 2548.73 & -11.87 & -3.43 & 12.36 \\
\hline 23 & -80.65 & 2497.45 & -97.68 & 2508.26 & 17.04 & -10.81 & 20.18 \\
\hline 24 & 29.07 & 2544.28 & 36.98 & 2541.10 & -7.91 & 3.18 & 8.52 \\
\hline 25 & 53.98 & 2531.47 & 53.27 & 2526.49 & 0.71 & 4.98 & 5.03 \\
\hline 26 & 41.95 & 2509.19 & 42.81 & 2524.11 & -0.87 & -14.92 & 14.94 \\
\hline 27 & 58.47 & 2523.17 & 52.48 & 2518.33 & 5.99 & 4.84 & 7.70 \\
\hline 28 & 49.61 & 2526.38 & 48.22 & 2541.24 & 1.40 & -14.86 & 14.93 \\
\hline 29 & 44.25 & 2506.82 & 44.78 & 2513.28 & -0.54 & -6.46 & 6.49 \\
\hline 30 & 46.77 & 2502.78 & 52.51 & 2518.02 & -5.74 & -15.25 & 16.29 \\
\hline 31 & 60.95 & 2524.16 & 60.75 & 2528.17 & 0.20 & -4.01 & 4.01 \\
\hline 32 & 53.27 & 2501.62 & 60.21 & 2490.34 & -6.94 & 11.28 & 13.24 \\
\hline 33 & 61.37 & 2518.41 & 58.52 & 2511.15 & 2.85 & 7.25 & 7.80 \\
\hline 34 & 62.93 & 2570.99 & 57.52 & 2572.76 & 5.41 & -1.77 & 5.69 \\
\hline 35 & -67.92 & 2502.39 & -87.76 & 2518.09 & 19.85 & -15.70 & 25.30 \\
\hline 36 & 161.74 & 2524.94 & 169.56 & 2527.09 & -7.82 & -2.15 & 8.11 \\
\hline 37 & -52.54 & 2536.23 & -76.53 & 2531.21 & 23.98 & 5.02 & 24.50 \\
\hline 38 & 66.55 & 2535.64 & 61.84 & 2546.20 & 4.71 & -10.56 & 11.56 \\
\hline 39 & 162.29 & 2520.38 & 178.36 & 2524.51 & -16.06 & -4.14 & 16.59 \\
\hline 40 & -79.09 & 2511.70 & -97.53 & 2510.21 & 18.44 & 1.49 & 18.50 \\
\hline 41 & 44.54 & 2526.46 & 53.12 & 2518.45 & -8.59 & 8.01 & 11.74 \\
\hline 42 & 70.05 & 2529.86 & 65.92 & 2553.12 & 4.14 & -23.26 & 23.62 \\
\hline 43 & 54.40 & 2464.35 & 48.71 & 2472.97 & 5.69 & -8.62 & 10.33 \\
\hline 44 & 195.16 & 2507.11 & 200.42 & 2504.84 & -5.25 & 2.27 & 5.72 \\
\hline 45 & 166.56 & 2511.01 & 180.74 & 2514.73 & -14.19 & -3.72 & 14.67 \\
\hline 46 & 176.99 & 2519.42 & 172.28 & 2520.67 & 4.71 & -1.26 & 4.88 \\
\hline 47 & 219.66 & 2504.34 & 217.49 & 2499.67 & 2.17 & 4.67 & 5.15 \\
\hline
\end{tabular}


Table 4.2: The CoM positions continued for Subject 1 for postures 48 through 94.

\begin{tabular}{|c|c|c|c|c|c|c|c|}
\hline \multirow[b]{2}{*}{ Postures } & \multicolumn{2}{|c|}{ Predicted CoM (mm) } & \multicolumn{2}{|c|}{ Measured CoM (mm) } & \multicolumn{2}{|c|}{ Error (mm) } & \multirow{2}{*}{$\frac{\text { Distance }(\mathrm{mm})}{d}$} \\
\hline & $x$ & $y$ & $x$ & $y$ & $\Delta x$ & $\Delta y$ & \\
\hline 48 & 51.06 & 2525.10 & 55.43 & 2521.04 & -4.37 & 4.07 & 5.97 \\
\hline 49 & 92.66 & 2504.17 & 77.81 & 2509.81 & 14.84 & -5.65 & 15.88 \\
\hline 50 & 54.51 & 2515.77 & 39.52 & 2525.52 & 14.99 & -9.75 & 17.88 \\
\hline 51 & 53.83 & 2560.28 & 58.18 & 2555.62 & -4.35 & 4.66 & 6.38 \\
\hline 52 & 58.96 & 2520.49 & 50.63 & 2515.65 & 8.34 & 4.83 & 9.64 \\
\hline $5 \overline{3}$ & 50.33 & 2523.69 & 57.59 & 2521.52 & -7.26 & 2.16 & 7.58 \\
\hline 54 & 207.01 & 2502.56 & 206.39 & 2511.02 & 0.62 & -8.46 & 8.48 \\
\hline 55 & -84.02 & 2513.64 & -100.85 & 2528.80 & 16.83 & -15.16 & 22.65 \\
\hline 56 & 51.89 & 2546.81 & 44.87 & 2536.55 & 7.03 & 10.27 & 12.44 \\
\hline 57 & -60.54 & 2496.80 & -78.22 & 2512.56 & 17.68 & -15.76 & 23.69 \\
\hline 58 & 50.94 & 2558.28 & 53.92 & 2549.80 & -2.98 & 8.47 & 8.98 \\
\hline 59 & -76.86 & 2515.93 & -86.62 & 2536.57 & 9.76 & -20.64 & 22.83 \\
\hline 60 & -79.51 & 2510.44 & -90.68 & 2518.66 & 11.17 & -8.22 & 13.87 \\
\hline 61 & -84.23 & 2497.80 & -92.13 & 2511.75 & 7.91 & -13.95 & 16.04 \\
\hline 62 & 47.46 & 2532.00 & 34.30 & 2538.34 & 13.16 & -6.34 & 14.60 \\
\hline 63 & 54.12 & 2529.69 & 55.57 & 2531.75 & -1.45 & -2.06 & 2.52 \\
\hline 64 & -84.61 & 2505.34 & -99.37 & 2525.12 & 14.76 & -19.78 & 24.68 \\
\hline 65 & 46.91 & 2527.14 & 56.06 & 2531.48 & -9.15 & -4.34 & 10.13 \\
\hline 66 & 64.49 & 2513.88 & 59.66 & 2510.23 & 4.83 & 3.65 & 6.05 \\
\hline 67 & 55.43 & 2485.22 & 66.82 & 2481.54 & -11.39 & 3.69 & 11.98 \\
\hline 68 & -92.99 & 2517.53 & -92.75 & 2512.69 & -0.24 & 4.84 & 4.85 \\
\hline 69 & 188.02 & 2492.79 & 199.30 & 2506.99 & -11.28 & -14.20 & 18.13 \\
\hline 70 & 164.91 & 2516.16 & 171.02 & 2521.43 & -6.11 & -5.27 & 8.07 \\
\hline 71 & 56.05 & 2540.79 & 40.93 & 2551.56 & 15.13 & -10.77 & 18.57 \\
\hline 72 & 68.98 & 2488.92 & 62.64 & 2502.10 & 6.35 & -13.18 & 14.63 \\
\hline 73 & 60.25 & 2562.44 & 58.81 & 2560.49 & 1.44 & 1.94 & 2.42 \\
\hline 74 & 113.98 & 2535.52 & 98.82 & 2544.70 & 15.16 & -9.18 & 17.72 \\
\hline 75 & 42.18 & 2525.30 & 26.95 & 2538.88 & 15.23 & -13.58 & 20.41 \\
\hline 76 & 40.04 & 2485.17 & 41.15 & 2473.10 & -1.11 & 12.07 & 12.12 \\
\hline 77 & 192.49 & 2489.50 & 197.13 & 2510.02 & -4.65 & -20.51 & 21.03 \\
\hline 78 & 59.31 & 2575.59 & 51.36 & 2574.25 & 7.95 & 1.34 & 8.06 \\
\hline 79 & 191.57 & 2531.08 & 197.62 & 2527.00 & -6.04 & 4.08 & 7.29 \\
\hline 80 & 39.47 & 2503.82 & 43.30 & 2502.70 & -3.83 & 1.12 & 3.99 \\
\hline 81 & 53.28 & 2527.53 & 47.47 & 2533.28 & 5.81 & -5.75 & 8.17 \\
\hline 82 & -89.84 & 2510.72 & -78.95 & 2522.06 & -10.89 & -11.33 & 15.72 \\
\hline 83 & 183.37 & 2522.32 & 187.93 & 2522.26 & -4.56 & 0.06 & 4.56 \\
\hline 84 & 69.88 & 2497.77 & 54.82 & 2508.18 & 15.06 & -10.41 & 18.31 \\
\hline 85 & 43.83 & 2527.61 & 49.80 & 2535.10 & -5.97 & -7.50 & 9.58 \\
\hline 86 & 25.34 & 2533.46 & 32.29 & 2525.37 & -6.95 & 8.09 & 10.67 \\
\hline 87 & 49.98 & 2530.64 & 52.55 & 2533.21 & -2.57 & -2.58 & 3.64 \\
\hline 88 & 40.80 & 2522.93 & 46.90 & 2522.08 & -6.10 & 0.85 & 6.16 \\
\hline 89 & 224.55 & 2518.18 & 216.39 & 2510.42 & 8.16 & 7.76 & 11.26 \\
\hline 90 & -36.41 & 2534.03 & -71.48 & 2533.65 & 35.07 & 0.38 & 35.07 \\
\hline 91 & 57.24 & 2528.92 & 59.12 & 2520.41 & -1.88 & 8.51 & 8.72 \\
\hline 92 & 7.50 & 2548.96 & 34.49 & 2550.46 & -26.99 & -1.50 & 27.03 \\
\hline 93 & 162.03 & 2525.38 & 165.41 & 2527.41 & -3.39 & -2.03 & 3.95 \\
\hline 94 & 26.67 & 2532.37 & 20.80 & 2522.18 & 5.87 & 10.19 & 11.76 \\
\hline Average $^{\S}$ & & & & & 8.32 & 8.04 & 12.64 \\
\hline RMSE & & & & & 10.53 & 9.72 & \\
\hline
\end{tabular}

${ }^{\S}$ For $\Delta x$ and $\Delta y$ the average is of the absolute values. 


\section{Experiment B}

In Experiment B, 107 static postures were recorded for Subject 2 in approximately 15 minutes. From this data, a total of 13 distinct postures were used as the training set to identify the SESC parameters for the articulated chain described in Section 3.2. As a result, the SESC of Subject 2 was constructed as seen in Fig. 4.5 and depicted by the green dashed lines where the terminal point is at the CoM.

To verify the accuracy of the method, the remaining test set of 94 postures were used to predict the CoM. Table 4.4 includes the results of the predicted and measured CoM obtained from the force plate. The CoM in the plane of the force plate is seen to have an average distance of $14.13 \mathrm{~mm}$ and an RMSE of $11.39 \mathrm{~mm}$ in the $x$ direction and $11.47 \mathrm{~mm}$ in the $y$ direction. The worst $\Delta x$ is 35.94 $\mathrm{mm}$ and the worst $\Delta y$ is $-34.61 \mathrm{~mm}$.

In a similar fashion to Experiment A, the effect of the number of postures to the method accuracy was observed by dividing the 107 postures into two sets. In the first set 77 postures were used as the training set to evaluate the SESC parameters of Subject 2. In the second set the remaining 30 postures were used as the testing set to evaluate the CoM. The average distance is plotted against the number of postures. Note that the average distance was $11.26 \mathrm{~mm}$ when using all of the 77 postures and the minimum error was $10.47 \mathrm{~mm}$ at the 25 postures as shown in Fig. 4.6. Modest improvement was seen as the number of modeling postures increased.

\section{Experiment C}

In Experiment C, 45 static postures were recorded for Subject 3 in approximately 5 minutes. For the first 13 postures the subject is instructed to approximately reproduce the 13 postures performed by Subject 2 as shown in Fig. 4.4. The 13 postures were used as the training set to identify the SESC parameters for the articulated chain described in Section 3.2. Thus, the SESC of Subject 3 

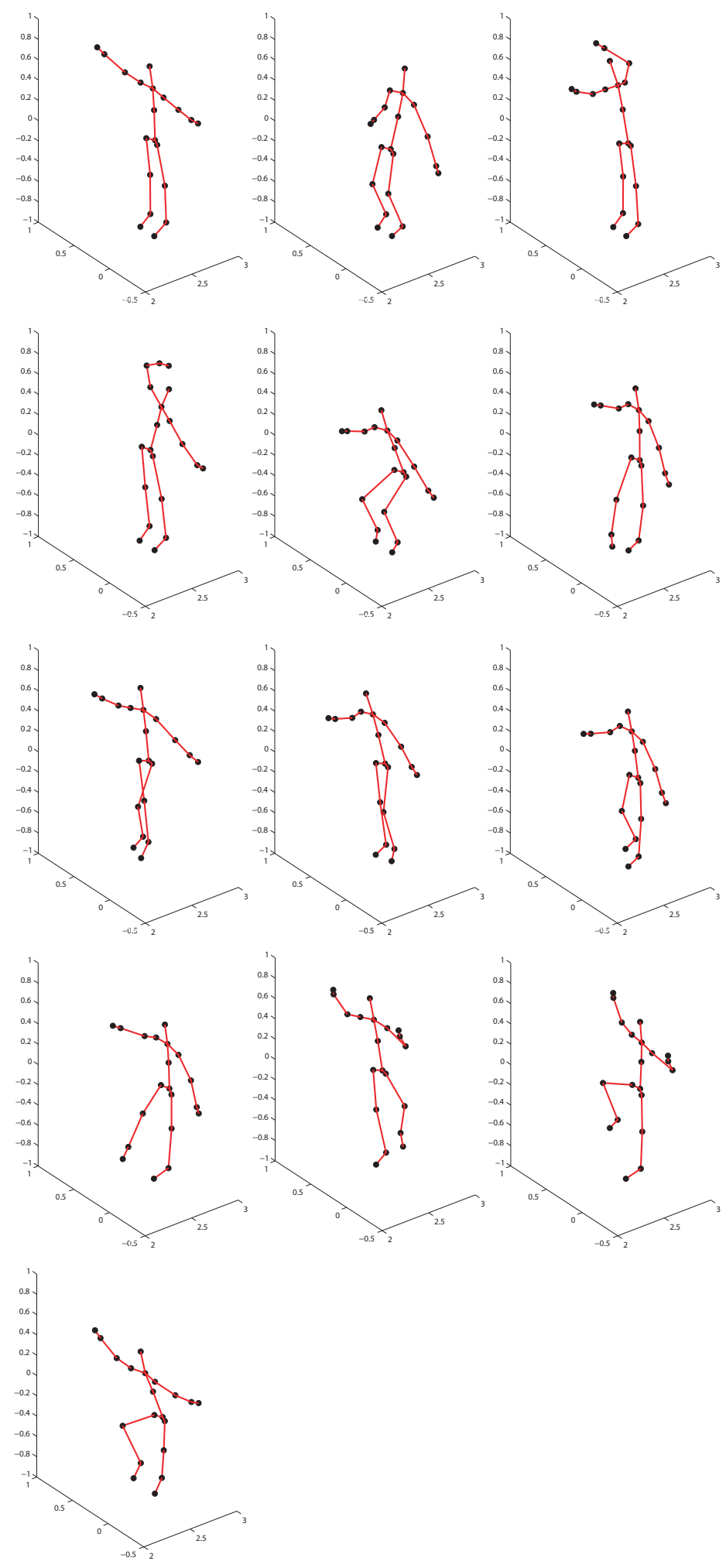

Figure 4.4: The 13 distinct postures performed by Subject 2 and Subject 3 during the SESC experimental construction. 


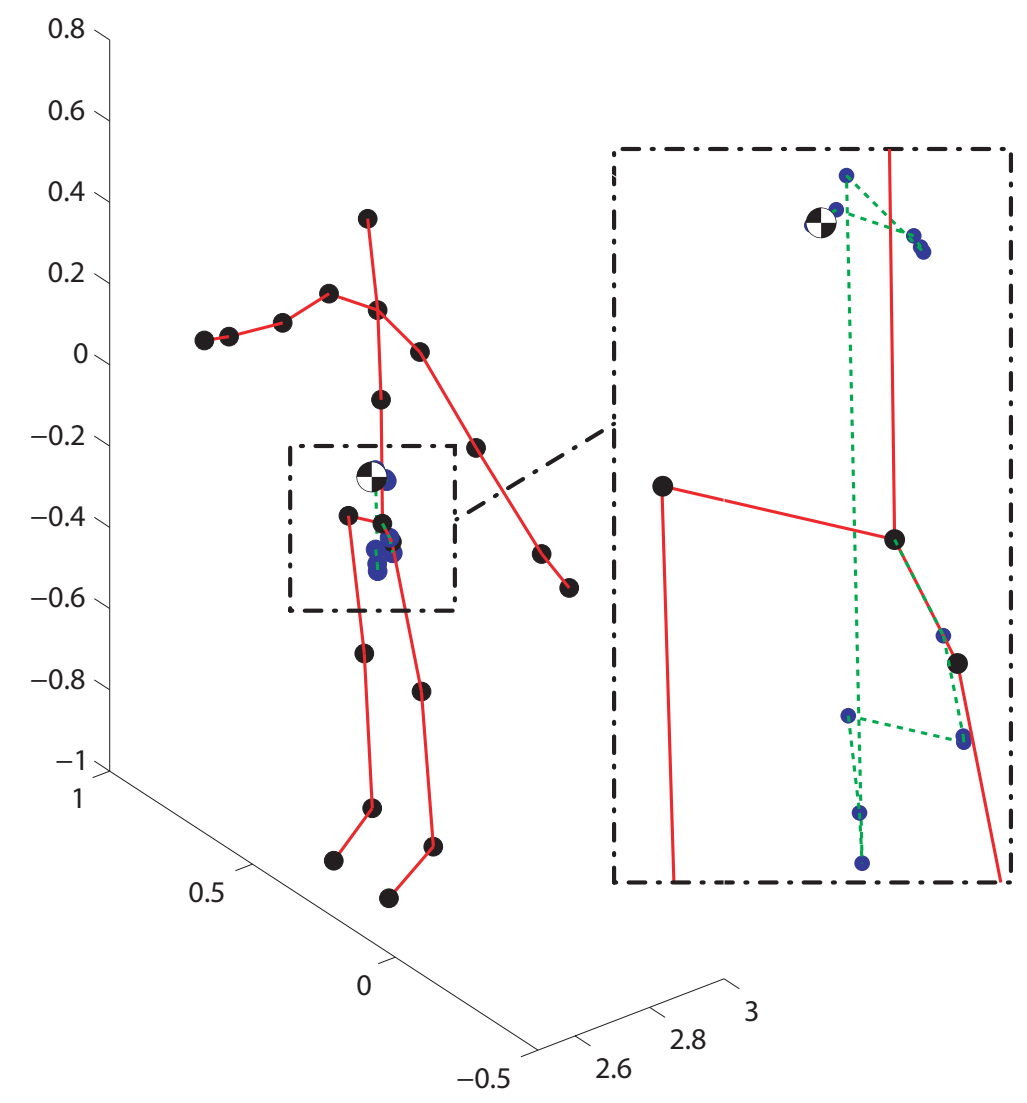

Figure 4.5: The SESC model of Subject 2 where the SESC, depicted by the green dashed lines, terminates at the CoM. 
Table 4.3: The CoM positions for Subject 2 as predicted by the node-based SESC method for the 94 postures using the data from the Kinect/WBB sensors. The table includes the deviations of the predicted CoM from the measured CoM for postures 1 through 47 .

\begin{tabular}{|c|c|c|c|c|c|c|c|}
\hline \multirow[b]{2}{*}{ Postures } & \multicolumn{2}{|c|}{ Predicted CoM (mm) } & \multicolumn{2}{|c|}{ Measured CoM $(\mathrm{mm})$} & \multicolumn{2}{|c|}{ Error $(\mathrm{mm})$} & \multirow{2}{*}{$\frac{\text { Distance }(\mathrm{mm})}{d}$} \\
\hline & $x$ & $y$ & $x$ & $y$ & $\Delta x$ & $\Delta y$ & \\
\hline 1 & 93.47 & 2508.51 & 95.28 & 2517.00 & -1.81 & -8.49 & 8.69 \\
\hline 2 & 92.19 & 2515.64 & 93.06 & 2518.36 & -0.87 & -2.72 & 2.86 \\
\hline 3 & 93.46 & 2517.89 & 93.99 & 2518.24 & -0.53 & -0.35 & 0.63 \\
\hline 4 & 208.36 & 2480.47 & 211.46 & 2496.03 & -3.10 & -15.57 & 15.87 \\
\hline 5 & -45.82 & 2517.37 & -53.24 & 2534.01 & 7.42 & -16.64 & 18.22 \\
\hline 6 & 131.06 & 2455.39 & 132.53 & 2458.44 & -1.47 & -3.05 & 3.39 \\
\hline 7 & 96.86 & 2483.36 & 92.00 & 2466.76 & 4.86 & 16.61 & 17.30 \\
\hline 8 & 87.03 & 2495.92 & 83.31 & 2485.61 & 3.72 & 10.31 & 10.96 \\
\hline 9 & -48.76 & 2519.41 & -47.50 & 2511.21 & -1.27 & 8.19 & 8.29 \\
\hline 10 & 231.46 & 2521.54 & 220.45 & 2515.25 & 11.01 & 6.29 & 12.68 \\
\hline 11 & 86.22 & 2486.67 & 89.72 & 2491.25 & -3.51 & -4.59 & 5.77 \\
\hline 12 & -55.09 & 2521.17 & -48.35 & 2516.29 & -6.75 & 4.88 & 8.32 \\
\hline 13 & 235.78 & 2533.05 & 222.90 & 2524.12 & 12.87 & 8.93 & 15.67 \\
\hline 14 & -89.70 & 2497.70 & -57.09 & 2500.18 & -32.60 & -2.48 & 32.70 \\
\hline 15 & 276.01 & 2485.47 & 240.06 & 2491.23 & 35.94 & -5.76 & 36.40 \\
\hline 16 & 208.83 & 2471.14 & 218.89 & 2471.74 & -10.06 & -0.61 & 10.08 \\
\hline 17 & 235.84 & 2500.66 & 238.61 & 2491.14 & -2.76 & 9.51 & 9.91 \\
\hline 18 & -79.27 & 2480.77 & -67.39 & 2487.31 & -11.88 & -6.54 & 13.56 \\
\hline 19 & -78.71 & 2466.96 & -77.12 & 2480.71 & -1.60 & -13.75 & 13.84 \\
\hline 20 & 224.42 & 2466.42 & 232.53 & 2467.52 & -8.11 & -1.11 & 8.18 \\
\hline 21 & 231.57 & 2459.00 & 235.38 & 2465.95 & -3.81 & -6.95 & 7.93 \\
\hline 22 & -57.37 & 2457.93 & -65.78 & 2472.03 & 8.42 & -14.10 & 16.42 \\
\hline 23 & -76.51 & 2435.48 & -70.64 & 2456.32 & -5.87 & -20.84 & 21.65 \\
\hline 24 & 98.18 & 2465.48 & 102.61 & 2470.12 & -4.43 & -4.64 & 6.42 \\
\hline 25 & 107.72 & 2473.33 & 109.24 & 2468.54 & -1.52 & 4.79 & 5.03 \\
\hline 26 & 102.37 & 2465.38 & 107.24 & 2460.11 & -4.87 & 5.27 & 7.17 \\
\hline 27 & 106.64 & 2514.97 & 90.81 & 2522.42 & 15.82 & -7.45 & 17.49 \\
\hline 28 & 88.65 & 2530.05 & 104.94 & 2528.88 & -16.29 & 1.17 & 16.33 \\
\hline 29 & 250.64 & 2521.23 & 242.68 & 2515.51 & 7.96 & 5.72 & 9.80 \\
\hline 30 & -67.56 & 2521.15 & -57.96 & 2523.09 & -9.61 & -1.94 & 9.80 \\
\hline 31 & 220.33 & 2528.85 & 216.96 & 2507.50 & 3.37 & 21.35 & 21.61 \\
\hline 32 & 244.25 & 2537.95 & 244.55 & 2512.36 & -0.30 & 25.58 & 25.59 \\
\hline 33 & 243.65 & 2510.02 & 226.57 & 2524.56 & 17.07 & -14.54 & 22.42 \\
\hline 34 & -59.46 & 2471.65 & -57.90 & 2468.22 & -1.57 & 3.44 & 3.78 \\
\hline 35 & -68.09 & 2522.49 & -57.60 & 2524.92 & -10.49 & -2.44 & 10.77 \\
\hline 36 & 229.78 & 2520.26 & 227.15 & 2522.11 & 2.63 & -1.85 & 3.22 \\
\hline 37 & 224.10 & 2483.13 & 225.16 & 2501.28 & -1.06 & -18.15 & 18.18 \\
\hline 38 & 248.62 & 2473.32 & 241.35 & 2489.13 & 7.27 & -15.80 & 17.40 \\
\hline 39 & 238.45 & 2476.85 & 240.94 & 2511.46 & -2.49 & -34.61 & 34.70 \\
\hline 40 & -72.62 & 2490.47 & -63.29 & 2502.47 & -9.33 & -12.00 & 15.20 \\
\hline 41 & -69.81 & 2482.29 & -78.44 & 2477.98 & 8.63 & 4.32 & 9.65 \\
\hline 42 & -64.65 & 2474.64 & -63.58 & 2488.98 & -1.07 & -14.34 & 14.38 \\
\hline 43 & 235.10 & 2523.85 & 232.09 & 2531.40 & 3.01 & -7.55 & 8.13 \\
\hline 44 & -77.35 & 2505.22 & -65.76 & 2510.87 & -11.59 & -5.65 & 12.89 \\
\hline 45 & 104.89 & 2467.53 & 107.97 & 2454.87 & -3.08 & 12.66 & 13.03 \\
\hline 46 & -78.80 & 2473.72 & -58.61 & 2493.91 & -20.19 & -20.20 & 28.56 \\
\hline 47 & 224.88 & 2493.57 & 235.10 & 2506.44 & -10.22 & -12.87 & 16.43 \\
\hline
\end{tabular}


Table 4.4: The CoM positions continued for Subject 2 for postures 48 through 94.

\begin{tabular}{|c|c|c|c|c|c|c|c|}
\hline \multirow[b]{2}{*}{ Postures } & \multicolumn{2}{|c|}{ Predicted CoM (mm) } & \multicolumn{2}{|c|}{ Measured CoM (mm) } & \multicolumn{2}{|c|}{ Error (mm) } & \multirow{2}{*}{$\frac{\text { Distance }(\mathrm{mm})}{d}$} \\
\hline & $x$ & $y$ & $x$ & $y$ & $\Delta x$ & $\Delta y$ & \\
\hline 48 & -75.42 & 2508.30 & -55.71 & 2509.79 & -19.71 & -1.49 & 19.77 \\
\hline 49 & -45.44 & 2537.17 & -49.30 & 2536.41 & 3.86 & 0.76 & 3.93 \\
\hline 50 & -83.97 & 2535.89 & -66.93 & 2532.21 & -17.04 & 3.68 & 17.43 \\
\hline 51 & 233.88 & 2537.13 & 251.17 & 2526.42 & -17.29 & 10.71 & 20.34 \\
\hline 52 & 233.17 & 2524.97 & 246.11 & 2509.35 & -12.94 & 15.62 & 20.28 \\
\hline $5 \overline{3}$ & 232.73 & 2513.63 & 249.69 & 2501.16 & -16.96 & 12.47 & 21.06 \\
\hline 54 & 267.42 & 2528.36 & 247.03 & 2524.16 & 20.38 & 4.20 & 20.81 \\
\hline 55 & 249.14 & 2534.51 & 238.27 & 2534.17 & 10.88 & 0.34 & 10.88 \\
\hline 56 & 246.44 & 2537.67 & 237.81 & 2524.35 & 8.62 & 13.33 & 15.87 \\
\hline 57 & -66.95 & 2536.26 & -48.28 & 2533.16 & -18.67 & 3.11 & 18.93 \\
\hline 58 & -93.59 & 2537.04 & -65.26 & 2532.59 & -28.33 & 4.45 & 28.67 \\
\hline 59 & -55.31 & 2542.39 & -47.44 & 2535.58 & -7.87 & 6.80 & 10.40 \\
\hline 60 & 242.31 & 2503.42 & 245.82 & 2520.98 & -3.51 & -17.56 & 17.90 \\
\hline 61 & 245.71 & 2476.45 & 234.41 & 2509.59 & 11.30 & -33.14 & 35.01 \\
\hline 62 & 226.98 & 2486.82 & 234.53 & 2520.12 & -7.54 & -33.30 & 34.14 \\
\hline 63 & -75.83 & 2496.22 & -64.71 & 2508.58 & -11.11 & -12.35 & 16.62 \\
\hline 64 & -74.17 & 2511.30 & -58.88 & 2519.14 & -15.29 & -7.84 & 17.18 \\
\hline 65 & -75.54 & 2493.04 & -69.87 & 2500.04 & -5.68 & -7.01 & 9.02 \\
\hline 66 & -59.90 & 2513.76 & -45.33 & 2518.35 & -14.57 & -4.59 & 15.28 \\
\hline 67 & 274.87 & 2518.44 & 252.23 & 2513.89 & 22.64 & 4.56 & 23.10 \\
\hline 68 & 82.94 & 2423.96 & 93.46 & 2422.24 & -10.52 & 1.72 & 10.66 \\
\hline 69 & 86.05 & 2428.72 & 92.08 & 2433.00 & -6.03 & -4.28 & 7.40 \\
\hline 70 & 92.35 & 2452.25 & 90.84 & 2447.55 & 1.51 & 4.70 & 4.94 \\
\hline 71 & 201.73 & 2491.59 & 195.80 & 2503.96 & 5.93 & -12.37 & 13.72 \\
\hline 72 & -5.69 & 2498.68 & 16.39 & 2499.52 & -22.08 & -0.84 & 22.10 \\
\hline $7 \overline{3}$ & 66.32 & 2571.50 & 78.15 & 2572.06 & -11.83 & -0.56 & 11.84 \\
\hline 74 & 146.07 & 2492.63 & 142.34 & 2490.47 & 3.73 & 2.16 & 4.31 \\
\hline 75 & 47.06 & 2508.16 & 64.55 & 2499.37 & -17.48 & 8.79 & 19.57 \\
\hline 76 & 93.17 & 2495.27 & 96.84 & 2490.68 & -3.67 & 4.59 & 5.88 \\
\hline 77 & 80.00 & 2444.24 & 97.17 & 2445.43 & -17.16 & -1.19 & 17.20 \\
\hline 78 & 119.70 & 2448.79 & 125.50 & 2463.30 & -5.80 & -14.51 & 15.63 \\
\hline 79 & 112.08 & 2443.65 & 103.11 & 2446.36 & 8.97 & -2.70 & 9.37 \\
\hline 80 & 92.32 & 2430.47 & 101.22 & 2431.28 & -8.90 & -0.81 & 8.94 \\
\hline 81 & 73.47 & 2448.72 & 90.48 & 2447.25 & -17.01 & 1.46 & 17.08 \\
\hline 82 & 89.32 & 2428.27 & 93.32 & 2442.02 & -3.99 & -13.75 & 14.32 \\
\hline 83 & 90.15 & 2430.52 & 94.68 & 2450.60 & -4.54 & -20.08 & 20.59 \\
\hline 84 & 85.91 & 2438.81 & 90.25 & 2444.16 & -4.35 & -5.35 & 6.89 \\
\hline 85 & 97.26 & 2468.49 & 104.04 & 2453.96 & -6.78 & 14.53 & 16.03 \\
\hline 86 & 94.06 & 2466.02 & 99.71 & 2456.37 & -5.65 & 9.65 & 11.18 \\
\hline 87 & 99.10 & 2466.08 & 100.89 & 2463.40 & -1.79 & 2.69 & 3.23 \\
\hline 88 & 106.68 & 2485.78 & 109.60 & 2476.85 & -2.92 & 8.92 & 9.39 \\
\hline 89 & 118.89 & 2471.64 & 117.08 & 2466.91 & 1.81 & 4.73 & 5.06 \\
\hline 90 & 120.65 & 2471.66 & 127.52 & 2470.92 & -6.87 & 0.74 & 6.91 \\
\hline 91 & 105.34 & 2468.84 & 105.31 & 2472.32 & 0.03 & -3.48 & 3.48 \\
\hline 92 & 248.13 & 2487.96 & 237.64 & 2497.61 & 10.49 & -9.65 & 14.26 \\
\hline 93 & -72.51 & 2533.88 & -61.90 & 2515.45 & -10.61 & 18.43 & 21.26 \\
\hline 94 & 91.53 & 2538.04 & 92.25 & 2534.98 & -0.71 & 3.06 & 3.14 \\
\hline Average $^{\S}$ & & & & & 8.82 & 8.72 & 14.13 \\
\hline RMSE & & & & & 11.39 & 11.47 & \\
\hline
\end{tabular}

${ }^{\S}$ For $\Delta x$ and $\Delta y$ the average is of the absolute values. 


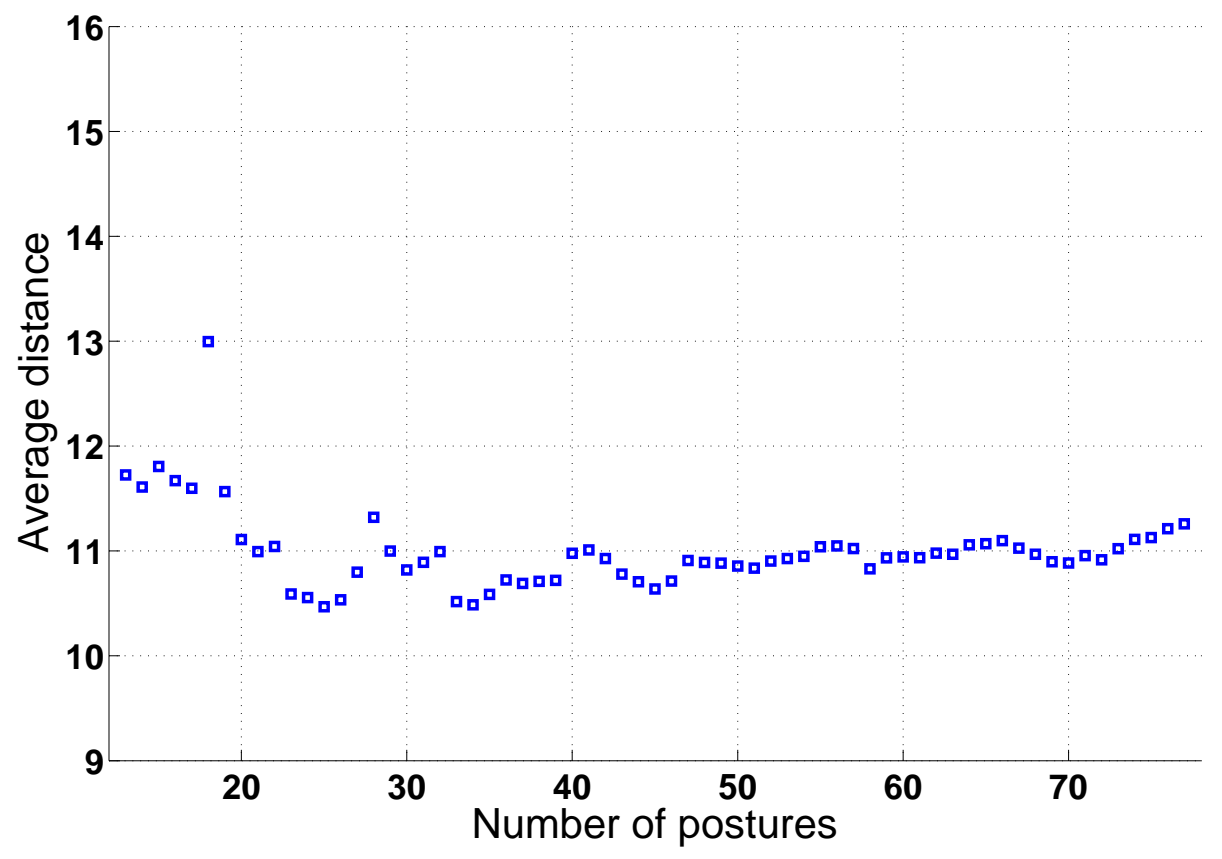

Figure 4.6: The average distance of the CoM for Subject 2 for the testing set of 30 postures as a function of the number of experimental postures.

was established and shown in Fig. 4.7 where the SESC is depicted by the green dashed lines and terminates at the CoM.

The verification of the method accuracy is evaluated by using the remaining 32 postures. These postures were used as the test set to predict the CoM and then comparing it to the measured CoM obtained from the force plate. The results of the predicted and measured CoM are in Tab. 4.5. The $\mathrm{CoM}$ in the plane of the force plate is seen to have an average distance of $12.06 \mathrm{~mm}$ and an RMSE of $7.42 \mathrm{~mm}$ in the $x$ direction and $11.47 \mathrm{~mm}$ in the $y$ direction. The worst $\Delta x$ is $18.29 \mathrm{~mm}$ and the worst $\Delta y$ is $-19.94 \mathrm{~mm}$.

The effect of the number of postures of Subject 3 to the method accuracy was observed by conducting two different tests. In the first test, 31 postures were used as the training set to evaluate the SESC parameters and the remaining 14 postures were used as the testing set to estimate the 


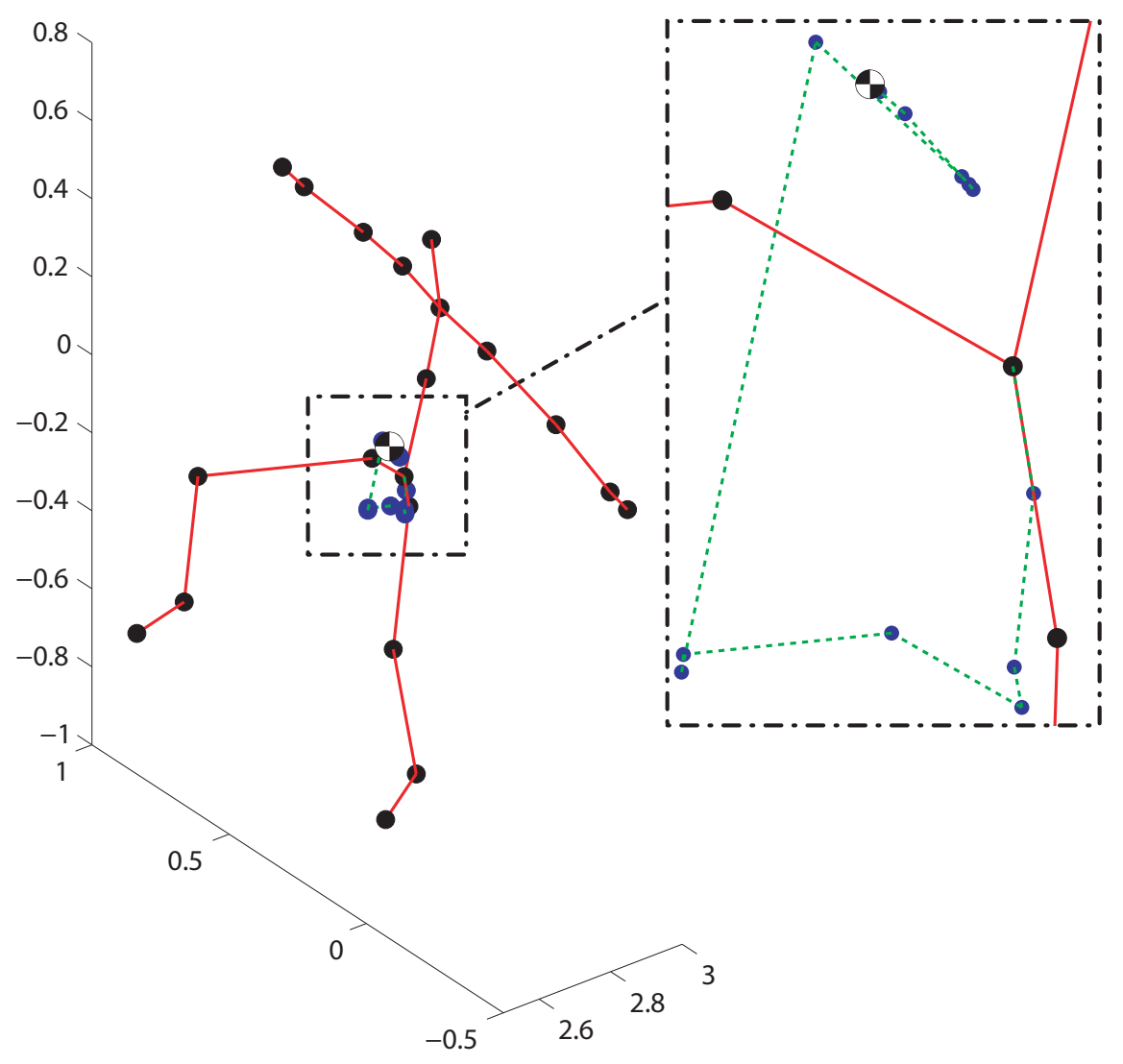

Figure 4.7: The SESC model of Subject 3. 
CoM. In the second test, a different set of 31 postures was used as the training set and the remaining 14 postures were used as the testing set. The average distance is plotted against the number of postures for both training sets and the results are shown in Fig. 4.8. Note that for the first training set, improvement was not observed as the number of postures increased. The average distance was $9.08 \mathrm{~mm}$ at 31 postures and the minimum distance was $8.64 \mathrm{~mm}$ at 30 postures as depicted in blue. For the second training set, in red, the results show the expected improvement in the average distance as the number of postures increases, with the average distance at 31 postures being 10.62 $\mathrm{mm}$.

For the three experiments, the average distances were found in the range $12.06 \mathrm{~mm}$ to 14.13 $\mathrm{mm}$. The worst $\Delta x$ and $\Delta y$ were $35.94 \mathrm{~mm}$ and $-34.61 \mathrm{~mm}$, respectively. Finally, increasing the size of the training data set was seen to correlate to improved predictions in Experiments A and B. In contrast, for Experiment $\mathrm{C}$, increasing the size of the first training data set was not seen to correlate to improved predictions. For the second training set, improvement was seen as the number of postures increased.

\subsection{Method Validation of The Donor Model using Kinect/WBB Sensors}

The donor model experiment introduced in this section consists of two parts. The first part, involves the construction of the SESC model for the male Subject 4 in a similar fashion to Experiments A through C. The second part, deals with the estimation of the SESC model of Subject 4 by relying on donor subjects. To validate the accuracy of the donor model, the CoM of Subject 4 is evaluated using the estimated SESC model from the donor subject, then compared to the predicted CoM of Subject 4 using the original SESC parameters.

In the first part of Experiment D, a total of 50 postures were recorded for Subject 4 in approximately 5 minutes. For the first 9 postures the subject was instructed to perform limited motion 
Table 4.5: The CoM positions for Subject 3 as predicted by the node-based SESC method for the 32 postures using the data from the Kinect/WBB sensors. The table includes the deviations of the predicted CoM from the measured CoM.

\begin{tabular}{|c|c|c|c|c|c|c|c|}
\hline \multirow[b]{2}{*}{ Postures } & \multicolumn{2}{|c|}{ Predicted CoM (mm) } & \multicolumn{2}{|c|}{ Measured CoM (mm) } & \multicolumn{2}{|c|}{ Error (mm) } & \multirow{2}{*}{$\frac{\text { Distance }(\mathrm{mm})}{d}$} \\
\hline & $x$ & $y$ & $x$ & $y$ & $\Delta x$ & $\Delta y$ & \\
\hline 1 & 44.98 & 2558.25 & 49.16 & 2540.98 & -4.18 & 17.27 & 17.77 \\
\hline 2 & 41.62 & 2551.11 & 45.35 & 2539.68 & -3.73 & 11.43 & 12.03 \\
\hline 3 & 45.61 & 2556.15 & 53.78 & 2562.07 & -8.17 & -5.92 & 10.09 \\
\hline 4 & 147.85 & 2511.94 & 162.56 & 2516.99 & -14.70 & -5.05 & 15.55 \\
\hline 5 & 159.15 & 2506.22 & 164.82 & 2521.02 & -5.67 & -14.80 & 15.85 \\
\hline 6 & 145.41 & 2518.15 & 158.84 & 2537.51 & -13.43 & -19.37 & 23.57 \\
\hline 7 & -43.37 & 2528.31 & -47.87 & 2517.46 & 4.50 & 10.84 & 11.74 \\
\hline 8 & 46.78 & 2574.96 & 48.35 & 2563.14 & -1.57 & 11.81 & 11.91 \\
\hline 9 & -74.15 & 2533.57 & -65.94 & 2536.45 & -8.21 & -2.87 & 8.70 \\
\hline 10 & 185.40 & 2502.94 & 181.37 & 2512.19 & 4.03 & -9.25 & 10.09 \\
\hline 11 & -34.60 & 2501.87 & -35.61 & 2515.02 & 1.01 & -13.15 & 13.19 \\
\hline 12 & 198.20 & 2507.55 & 198.37 & 2523.29 & -0.18 & -15.74 & 15.74 \\
\hline 13 & 136.45 & 2548.65 & 125.14 & 2568.59 & 11.31 & -19.94 & 22.93 \\
\hline 14 & -5.52 & 2584.10 & 10.40 & 2590.89 & -15.92 & -6.79 & 17.31 \\
\hline 15 & -71.28 & 2507.38 & -67.14 & 2511.64 & -4.14 & -4.26 & 5.94 \\
\hline 16 & 174.79 & 2523.82 & 170.73 & 2539.63 & 4.06 & -15.82 & 16.33 \\
\hline 17 & 51.48 & 2533.55 & 55.45 & 2528.16 & -3.96 & 5.39 & 6.69 \\
\hline 18 & 80.60 & 2493.72 & 62.31 & 2509.90 & 18.29 & -16.18 & 24.42 \\
\hline 19 & 41.00 & 2515.23 & 52.57 & 2533.63 & -11.57 & -18.40 & 21.74 \\
\hline 20 & -55.48 & 2525.72 & -58.72 & 2521.32 & 3.24 & 4.40 & 5.46 \\
\hline 21 & 172.23 & 2507.44 & 166.30 & 2527.28 & 5.94 & -19.84 & 20.71 \\
\hline 22 & 33.47 & 2554.58 & 26.59 & 2548.87 & 6.88 & 5.71 & 8.95 \\
\hline 23 & 20.43 & 2559.17 & 25.88 & 2542.59 & -5.45 & 16.59 & 17.46 \\
\hline 24 & 49.64 & 2516.82 & 52.14 & 2517.71 & -2.50 & -0.90 & 2.66 \\
\hline 25 & -53.11 & 2529.69 & -51.78 & 2530.04 & -1.33 & -0.35 & 1.37 \\
\hline 26 & 174.39 & 2546.09 & 177.53 & 2534.03 & -3.14 & 12.06 & 12.46 \\
\hline 27 & 80.31 & 2559.28 & 77.01 & 2555.85 & 3.29 & 3.43 & 4.76 \\
\hline 28 & 47.97 & 2574.67 & 53.27 & 2571.90 & -5.31 & 2.77 & 5.99 \\
\hline 29 & -69.90 & 2521.52 & -71.32 & 2525.72 & 1.43 & -4.20 & 4.44 \\
\hline 30 & 161.66 & 2519.08 & 160.63 & 2522.34 & 1.03 & -3.26 & 3.42 \\
\hline 31 & 47.22 & 2491.83 & 53.08 & 2486.50 & -5.86 & 5.34 & 7.92 \\
\hline 32 & 48.25 & 2571.47 & 45.15 & 2563.29 & 3.10 & 8.18 & 8.75 \\
\hline Average $^{\S}$ & & & & & 5.85 & 9.73 & 12.06 \\
\hline RMSE & & & & & 7.42 & 11.47 & \\
\hline
\end{tabular}

\footnotetext{
${ }^{\S}$ For $\Delta x$ and $\Delta y$ the average is of the absolute values.
} 


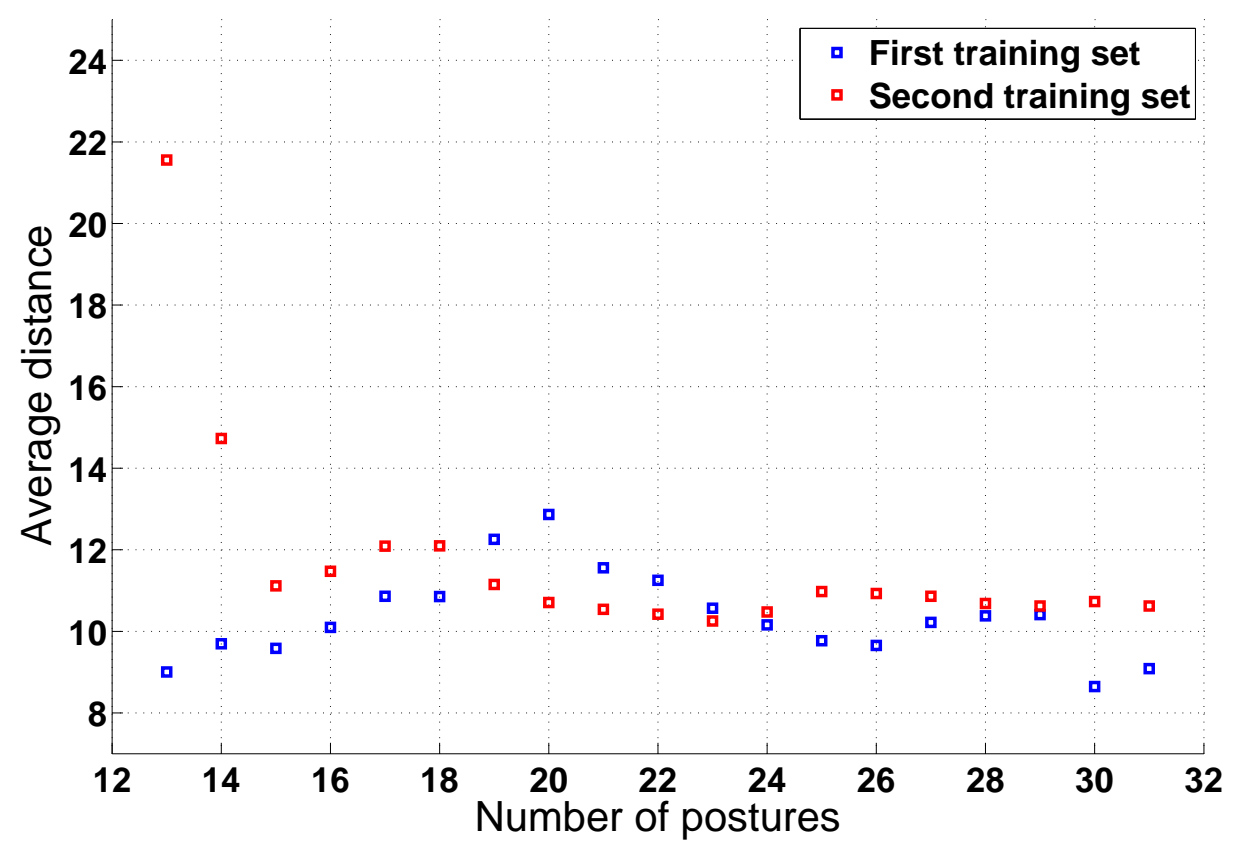

Figure 4.8: The average distance of the CoM for Subject 3 as a function of the number of experimental postures using the two different training and testing sets.

postures (i.e. like an elderly person suffering from Parkinson's Disease) as shown in Fig. 4.10. Note that the first seven limited motion postures were not used in the first part of the experiment and are saved for the donor model case in the second part of Experiment D. Then, the subject was instructed to approximately reproduce 11 postures from those performed by Subject 2 and Subject 3, in Fig. 4.4. The remaining limited motion postures (two postures) and the 11 postures were combined and used as the training set to identify the SESC parameters for Subject 4. As a result, the SESC of Subject 4 was constructed and is depicted by the green dashed lines in Fig. 4.9.

To verify the accuracy of the data acquired in part one, the remaining 30 postures were used as the testing set to evaluate the CoM. Table 4.6 includes the results of the predicted CoM and the measured CoM. The CoM in the plane of the force plate is seen to have an average distance of 13.78 


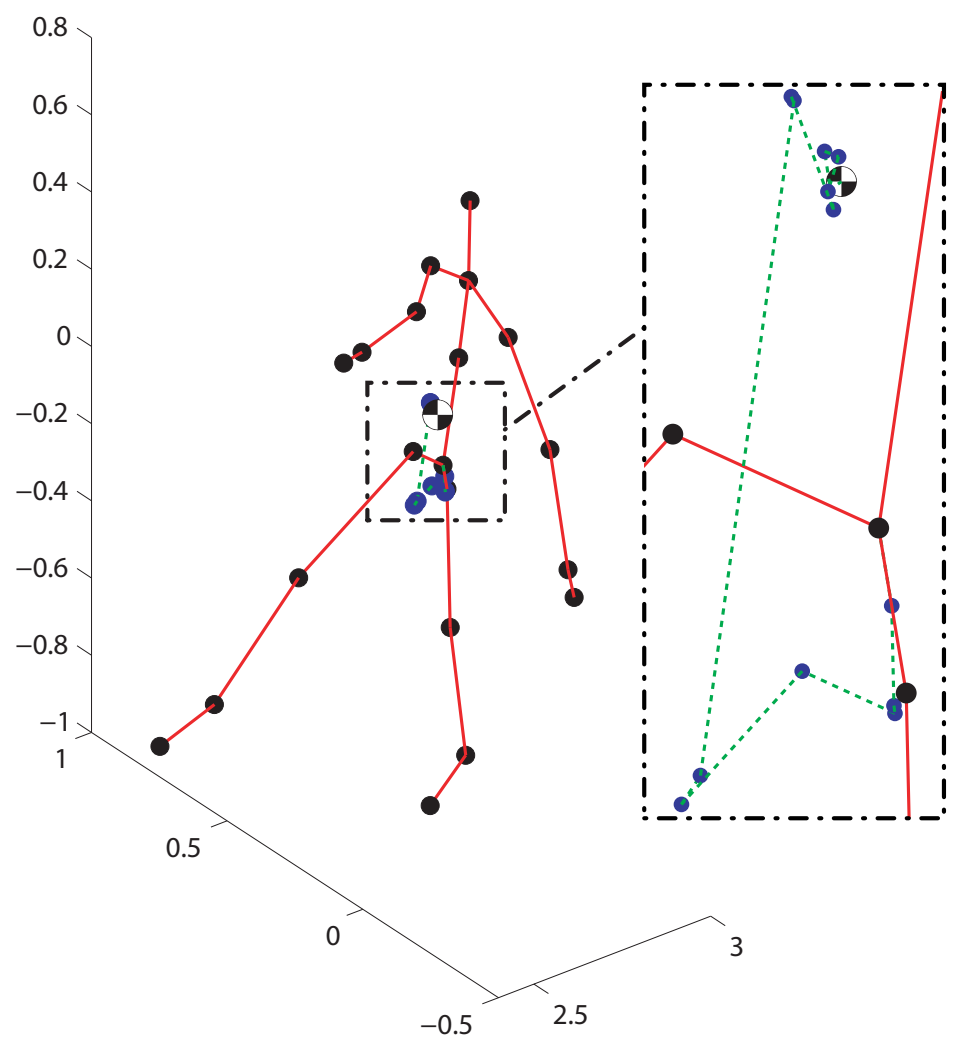

Figure 4.9: The SESC model of subject 4. 
$\mathrm{mm}$ and an RMSE of $9.88 \mathrm{~mm}$ in the $x$ direction and 11.65 in the $y$ direction. The worst $\Delta x$ is $-22.56 \mathrm{~mm}$ and the worst $\Delta y$ is $-23.67 \mathrm{~mm}$.

In the second part of Experiment D, the transferability of the SESC parameters among subjects with similar body build (i.e. height, weight, and body shape) was investigated. Three of the seven limited motion postures performed by Subject 4, saved from the first part of Experiment D, were paired with the SESC parameters of Subject 2 (Donor 1) and also paired with the SESC parameters of Subject 3 (Donor 2) to estimate the SESC parameters of Subject 4 as described in Section 3.3. Note that this process will result with two estimated sets of SESC parameters for Subject 4.

To validate the accuracy of the donor model, the remaining four limited motion postures were used as the testing set to evaluate the CoM. The CoM is evaluated by using the SESC parameters estimated from Donor 1 and from Donor 2. The validation is done by comparing the predicted CoM (obtained by using the SESC parameters estimated from Donor 1 and Donor 2) and the measured CoM via the force plate. The CoM in the plane of the force plate is seen to have an average distance of $5.83 \mathrm{~mm}$ and an RMSE of $3.80 \mathrm{~mm}$ in the $x$ direction and $4.60 \mathrm{~mm}$ in the $y$ direction when using the SESC parameters estimated from Donor 1 as depicted in Tab. 4.8. Moreover, when using the SESC parameters estimated from Donor 2, the CoM in the plane of the force plate is seen to have an average distance of $6.23 \mathrm{~mm}$ and an RMSE of $4.40 \mathrm{~mm}$ in the $x$ direction and $4.60 \mathrm{~mm}$ in the $y$ direction as depicted in Tab. 4.9. In contrast, using the original SESC parameters from Subject 4, the CoM in the plane of the force plate is seen to have an average distance of $6.29 \mathrm{~mm}$ and an RMSE of $4.80 \mathrm{~mm}$ in the $x$ direction and $4.20 \mathrm{~mm}$ in the $y$ direction as depicted in Tab. 4.7. Table 4.10 summarizes these results. Note that the average distance produced by Donor 1 was $6.23 \mathrm{~mm}$ and the distance obtained by Subject 4 is $6.29 \mathrm{~mm}$. The distance was close due to the similarity between Donor 1 with a height of 1.8 meters and Subject 4 at 1.79 meters tall. 
Table 4.6: The CoM positions for Subject 4 as predicted by the node-based SESC method for the 30 postures using the data from the Kinect/WBB sensors. The table includes the deviations of the predicted CoM from the measured CoM.

\begin{tabular}{|c|c|c|c|c|c|c|c|}
\hline \multirow[b]{2}{*}{ Postures } & \multicolumn{2}{|c|}{ Predicted CoM (mm) } & \multicolumn{2}{|c|}{ Measured CoM (mm) } & \multicolumn{2}{|c|}{ Error $(\mathrm{mm})$} & \multirow{2}{*}{$\frac{\text { Distance }(\mathrm{mm})}{d}$} \\
\hline & $x$ & $y$ & $x$ & $y$ & $\Delta x$ & $\Delta y$ & \\
\hline 1 & 199.89 & 2551.31 & 198.51 & 2545.84 & 1.38 & 5.47 & 5.64 \\
\hline 2 & 82.14 & 2541.04 & 79.87 & 2561.21 & 2.27 & -20.17 & 20.30 \\
\hline 3 & 198.27 & 2514.52 & 205.83 & 2526.26 & -7.56 & -11.75 & 13.97 \\
\hline 4 & -84.07 & 2488.97 & -83.19 & 2497.27 & -0.89 & -8.30 & 8.35 \\
\hline 5 & 57.13 & 2547.28 & 61.08 & 2547.01 & -3.95 & 0.27 & 3.96 \\
\hline 6 & 106.81 & 2580.58 & 90.46 & 2573.60 & 16.35 & 6.99 & 17.78 \\
\hline 7 & 27.06 & 2563.64 & 49.62 & 2563.78 & -22.56 & -0.14 & 22.56 \\
\hline 8 & 151.27 & 2566.25 & 150.57 & 2564.26 & 0.70 & 2.00 & 2.11 \\
\hline 9 & -13.99 & 2546.98 & -0.69 & 2531.96 & -13.30 & 15.03 & 20.07 \\
\hline 10 & -103.40 & 2524.58 & -95.17 & 2531.83 & -8.23 & -7.25 & 10.97 \\
\hline 11 & 198.41 & 2538.79 & 200.20 & 2530.55 & -1.79 & 8.25 & 8.44 \\
\hline 12 & 70.95 & 2520.76 & 80.01 & 2531.35 & -9.06 & -10.59 & 13.94 \\
\hline 13 & 188.41 & 2550.87 & 196.35 & 2560.40 & -7.94 & -9.53 & 12.41 \\
\hline 14 & -90.42 & 2498.24 & -86.53 & 2519.79 & -3.89 & -21.54 & 21.89 \\
\hline 15 & 193.34 & 2569.33 & 191.45 & 2562.98 & 1.89 & 6.35 & 6.62 \\
\hline 16 & -99.69 & 2534.80 & -94.10 & 2531.05 & -5.58 & 3.75 & 6.73 \\
\hline 17 & 213.38 & 2515.36 & 216.66 & 2539.03 & -3.29 & -23.67 & 23.90 \\
\hline 18 & -106.50 & 2499.91 & -95.35 & 2521.90 & -11.15 & -21.99 & 24.65 \\
\hline 19 & 113.47 & 2545.36 & 108.31 & 2560.26 & 5.16 & -14.90 & 15.77 \\
\hline 20 & 3.91 & 2554.32 & 12.64 & 2548.71 & -8.73 & 5.61 & 10.38 \\
\hline 21 & -103.18 & 2484.80 & -87.99 & 2490.41 & -15.19 & -5.62 & 16.19 \\
\hline 22 & 207.02 & 2508.76 & 201.47 & 2524.75 & 5.55 & -15.99 & 16.93 \\
\hline 23 & 77.67 & 2563.60 & 74.86 & 2568.94 & 2.81 & -5.34 & 6.03 \\
\hline 24 & 66.95 & 2555.95 & 76.66 & 2558.46 & -9.71 & -2.51 & 10.03 \\
\hline 25 & 41.28 & 2549.52 & 63.13 & 2560.13 & -21.86 & -10.61 & 24.29 \\
\hline 26 & 43.36 & 2557.11 & 45.01 & 2564.68 & -1.66 & -7.57 & 7.75 \\
\hline 27 & 75.10 & 2494.70 & 70.05 & 2484.67 & 5.04 & 10.03 & 11.23 \\
\hline 28 & 107.78 & 2514.26 & 101.87 & 2502.69 & 5.91 & 11.57 & 12.99 \\
\hline 29 & 29.98 & 2518.81 & 34.00 & 2506.53 & -4.02 & 12.28 & 12.92 \\
\hline 30 & 165.09 & 2558.07 & 186.80 & 2546.57 & -21.71 & 11.49 & 24.56 \\
\hline Average $^{\S}$ & & & & & 7.64 & 9.88 & 13.78 \\
\hline RMSE & & & & & 9.88 & 11.65 & \\
\hline
\end{tabular}

${ }^{\S}$ For $\Delta x$ and $\Delta y$ the average is of the absolute values. 

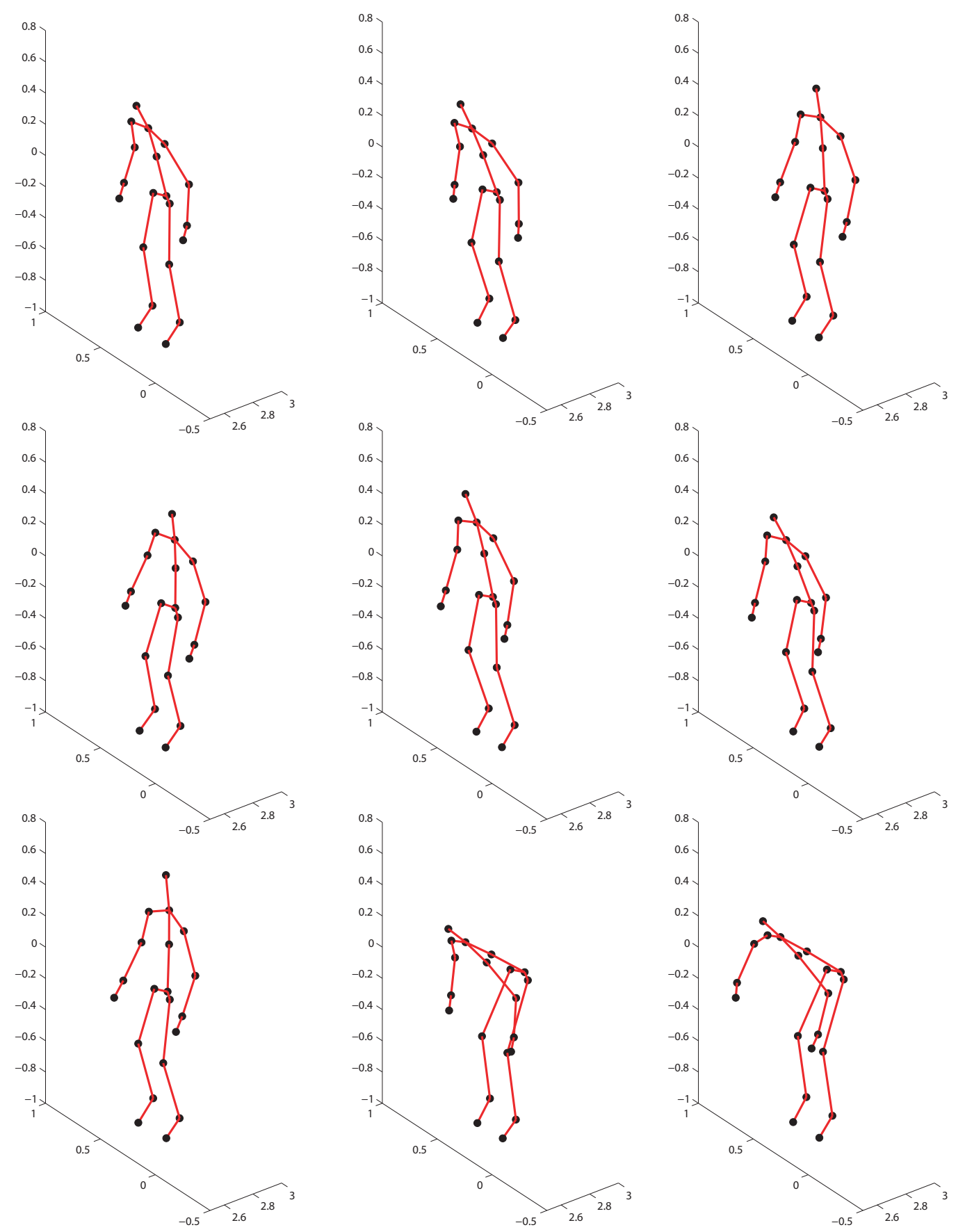

Figure 4.10: The nine limited motion postures of Subject 4 during the SESC experimental construction. 
Table 4.7: The CoM positions for Subject 4 as predicted by the node-based SESC method for the four limited motion postures using the data from the Kinect/WBB sensors. The table includes the deviations of the predicted CoM from the measured CoM.

\begin{tabular}{|c|c|c|c|c|c|c|}
\hline \multirow[b]{2}{*}{ Postures } & \multicolumn{2}{|c|}{ Predicted CoM (mm) } & \multicolumn{2}{|c|}{ Measured CoM (mm) } & \multicolumn{2}{|c|}{ Error (mm) } \\
\hline & $x$ & $y$ & $x$ & $y$ & $\Delta x$ & $\Delta y$ \\
\hline 1 & -36.79 & 2571.11 & -31.56 & 2567.42 & -5.24 & 3.69 \\
\hline 2 & 150.04 & 2545.31 & 156.55 & 2543.91 & -6.51 & 1.40 \\
\hline 3 & 87.35 & 2475.43 & 91.49 & 2476.27 & -4.14 & -0.84 \\
\hline 4 & 78.72 & 2617.12 & 76.26 & 2609.65 & 2.46 & 7.46 \\
\hline
\end{tabular}

Table 4.8: The CoM positions for Subject 4 as predicted by the node-based SESC donor model, from Donor 1, for the four limited motion postures using the data from the Kinect/WBB sensors. The table includes the deviations of the predicted CoM from the measured CoM.

\begin{tabular}{lcccccccc}
\hline \hline & \multicolumn{2}{c}{ Predicted CoM $(\mathrm{mm})$} & & \multicolumn{2}{c}{ Measured CoM $(\mathrm{mm})$} & & \multicolumn{2}{c}{ Error $(\mathrm{mm})$} \\
\cline { 2 - 3 } Postures & $x$ & $y$ & & $x$ & $y$ & & $\Delta x$ & $\Delta y$ \\
\hline 1 & -28.72 & 2572.31 & & -31.56 & 2567.42 & & 2.83 & 4.89 \\
2 & 153.76 & 2547.19 & & 156.55 & 2543.91 & & -2.79 & 3.29 \\
3 & 89.01 & 2483.08 & & 91.49 & 2476.27 & & -2.48 & 6.81 \\
4 & 82.19 & 2608.24 & & 76.26 & 2609.65 & & 5.94 & -1.42 \\
\hline \hline
\end{tabular}

Table 4.9: The CoM positions for Subject 4 as predicted by the node-based SESC donor model, from Donor 2, for the four limited motion postures using the data from the Kinect/WBB sensors. The table includes the deviations of the predicted CoM from the measured CoM.

\begin{tabular}{|c|c|c|c|c|c|c|}
\hline \multirow[b]{2}{*}{ Postures } & \multicolumn{2}{|c|}{ Predicted CoM (mm) } & \multicolumn{2}{|c|}{ Measured CoM (mm) } & \multicolumn{2}{|c|}{ Error (mm) } \\
\hline & $x$ & $y$ & $x$ & $y$ & $\Delta x$ & $\Delta y$ \\
\hline 1 & -28.14 & 2573.09 & -31.56 & 2567.42 & 3.41 & 5.67 \\
\hline 2 & 153.98 & 2547.01 & 156.55 & 2543.91 & -2.57 & 3.10 \\
\hline 3 & 88.83 & 2482.80 & 91.49 & 2476.27 & -2.66 & 6.52 \\
\hline 4 & 83.41 & 2608.59 & 76.26 & 2609.65 & 7.15 & -1.06 \\
\hline
\end{tabular}


Table 4.10: The average error and the RMSE of the CoM positions for Subject 4 as predicted by the node-based SESC versus the one predicted by the donor model, from Donor 1 and Donor 2, for the four limited motion postures using the data from the Kinect/WBB sensors.

\begin{tabular}{|c|c|c|c|c|c|}
\hline \multirow[b]{2}{*}{ Case } & \multicolumn{2}{|c|}{${ }^{\S}$ Average Error $(\mathrm{mm})$} & \multicolumn{2}{|c|}{$\mathrm{RMSE}(\mathrm{mm})$} & \multirow{2}{*}{$\frac{\text { Distance }(\mathrm{mm})}{d}$} \\
\hline & $\Delta x$ & $\Delta y$ & $\Delta x$ & $\Delta y$ & \\
\hline Subject 4 & 4.58 & 3.34 & 4.80 & 4.20 & 6.29 \\
\hline Donor 1 & 3.51 & 4.10 & 3.80 & 4.60 & 5.83 \\
\hline Donor 2 & 3.94 & 4.08 & 4.40 & 4.60 & 6.23 \\
\hline
\end{tabular}

$\S^{\S}$ Average of the absolute value for $\Delta x$ and $\Delta y$.

\subsection{Method Validation Where $\mathbf{A}$ and $\vec{d}$ are Unknown}

The node-based SESC model can be constructed by first calibrating the Kinect and the WBB as seen in the previous sections. The calibration procedure drastically reduces the number of postures required to construct the SESC. However, if the number of postures is not an issue, the node-base SESC model can be constructed without calibrating the two sensors, hence without the knowledge of $\mathbf{A}$ and $\vec{d}$, as detailed in Section 3.6. The $\mathbf{A}$ and the projection of $\vec{d}$ obtained by the method are

$$
\mathbf{A}=\left[\begin{array}{llr}
0.0595 & -0.9967 & -0.0785 \\
0.9981 & -0.0170 & -0.0100 \\
0.0147 & -0.0789 & 0.9938
\end{array}\right]
$$

and

$$
\overrightarrow{d_{p}}=\left\{\begin{array}{c}
-13.25 \\
2419
\end{array}\right\}
$$

corresponding to the experimental set up shown in Fig. 2.2. Note that $\mathbf{A}$ and the projection of $\vec{d}$ matched observation. Relevant details about the validation of the method is now presented.

\subsubsection{Method Validation using Kinect/WBB Sensors}

In Experiment E, a total of 106 static postures were recorded for Subject 5 in approximately 15 minutes. From this data, 96 postures were used as the training set to identify the SESC parameters 
for the articulated chain described in Section 3.2. The resulting SESC model is depicted in Fig. 4.11 where the terminal point is the CoM.

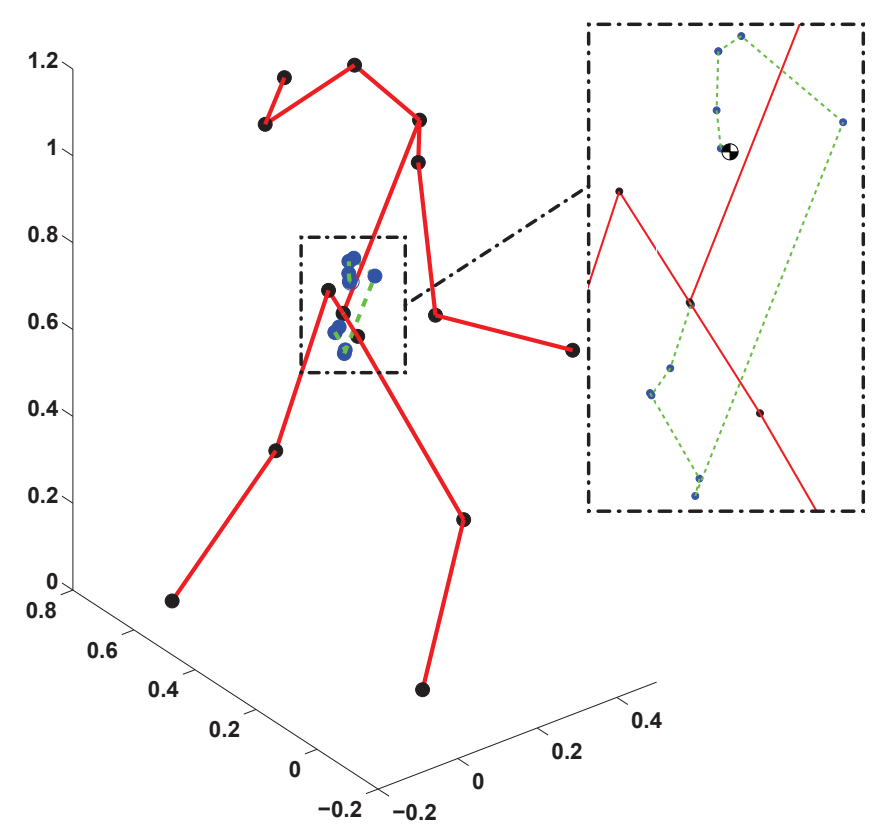

Figure 4.11: The SESC model of Subject 5.

In order to verify the accuracy of the method, the remaining ten postures were used as the testing set to evaluate the CoM. The results of the predicted CoM and the measured CoM via the force plate are depicted in Tab. 4.11. The CoM in the plane of the force plate is seen to have an average distance of about $5.87 \mathrm{~mm}$ and an RMSE of $5.20 \mathrm{~mm}$ in the $x$ direction and $4.55 \mathrm{~mm}$ in the $y$ direction. The worst $\Delta x$ is $12.80 \mathrm{~mm}$ and the worst $\Delta y$ is $8.74 \mathrm{~mm}$. The average distance is plotted against the number of postures and the results are shown in Fig. 4.12. Note that having $\mathbf{A}$ and $\vec{d}$ beforehand requires, theoretically, 13 postures for a unique solution to (3.15). However, this method requires 42 postures for a unique solution to (3.48) in which $\mathbf{A}$ is also determined. The results show the expected improvement as the number of postures increases. 


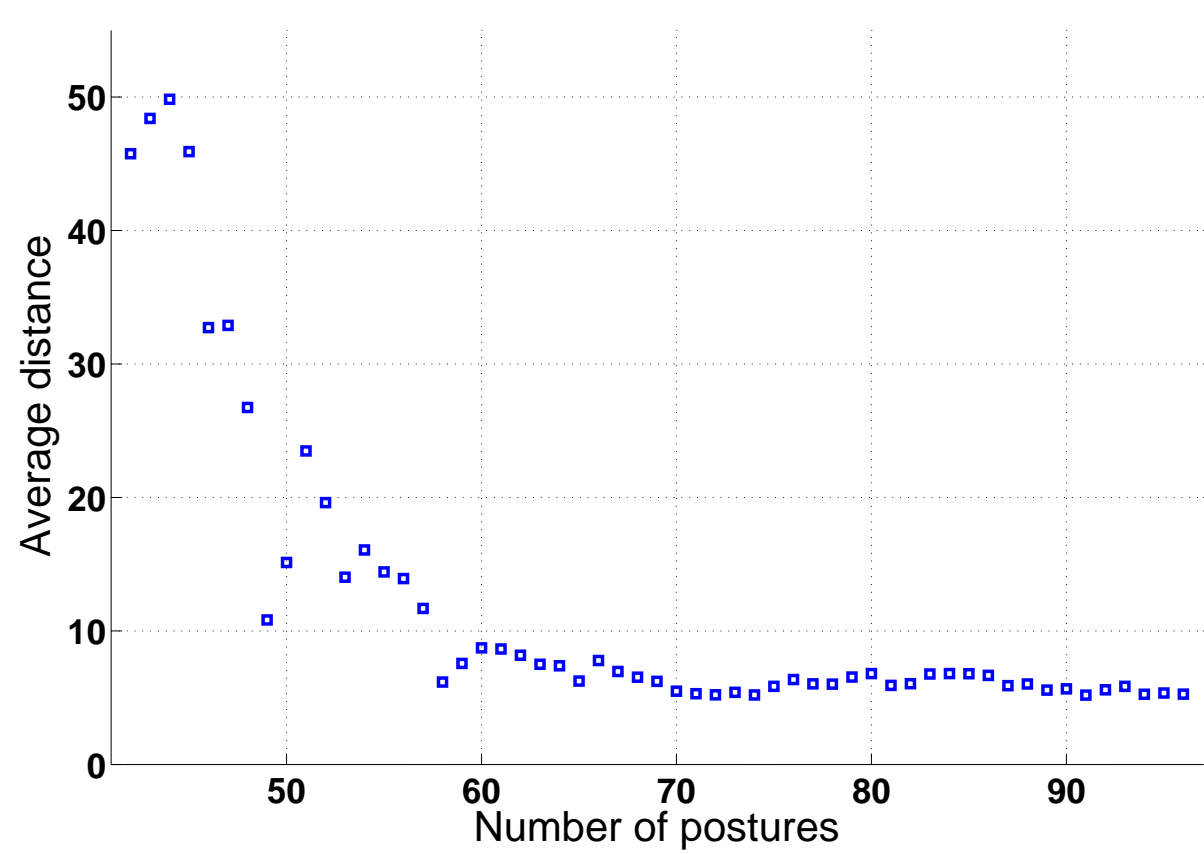

Figure 4.12: The average distance of the CoM for Subject 5 for the testing set of ten postures as a function of the number of experimental postures.

Table 4.11: The CoM positions for Subject 5 as predicted by the node-based SESC method for the ten postures using the data from the Kinect/WBB sensors. The table includes the deviations of the predicted CoM from the measured CoM.

\begin{tabular}{|c|c|c|c|c|c|c|c|}
\hline \multirow[b]{2}{*}{ Postures } & \multicolumn{2}{|c|}{ Predicted CoM (mm) } & \multicolumn{2}{|c|}{ Measured CoM (mm) } & \multicolumn{2}{|c|}{ Error $(\mathrm{mm})$} & \multirow{2}{*}{$\frac{\text { Distance }(\mathrm{mm})}{d}$} \\
\hline & $x$ & $y$ & $x$ & $y$ & $\Delta x$ & $\Delta y$ & \\
\hline 1 & 14.40 & 2388.00 & 1.53 & 2377.00 & 12.80 & 8.74 & 15.50 \\
\hline 2 & 18.00 & 2388.00 & 13.80 & 2377.00 & 4.17 & 6.05 & 7.35 \\
\hline 3 & 10.40 & 2388.00 & 3.86 & 2388.00 & 6.52 & 2.98 & 7.17 \\
\hline 4 & -1.47 & 2411.00 & -2.45 & 2422.00 & 0.98 & -6.37 & 6.44 \\
\hline 5 & 6.20 & 2411.00 & 3.41 & 2411.00 & 2.80 & -0.46 & 2.84 \\
\hline 6 & 8.19 & 2400.00 & 3.30 & 2400.00 & 4.89 & -0.64 & 4.93 \\
\hline 7 & 0.46 & 2400.00 & -2.65 & 2400.00 & 3.11 & -2.71 & 4.13 \\
\hline 8 & 7.81 & 2388.00 & 7.26 & 2399.00 & 0.54 & -2.52 & 2.58 \\
\hline 9 & -1.36 & 2399.00 & -1.38 & 2399.00 & 0.02 & 2.50 & 2.50 \\
\hline 10 & 1.63 & 2455.00 & 3.50 & 2455.00 & -1.87 & 4.93 & 5.27 \\
\hline Average $^{\S}$ & & & & & 3.77 & 3.79 & 5.87 \\
\hline RMSE & & & & & 5.20 & 4.55 & \\
\hline
\end{tabular}

${ }^{\S}$ For $\Delta x$ and $\Delta y$ the average is of the absolute values. 


\subsubsection{Method Validation using VICON/AMTI-OR6 Sensors With a Static Body In the Work-space}

In Experiment F, Fig. 4.13, Subject 6 went through the process of the placement of the 35 passive markers, following the Plug-in-Gait (PiG) template, to define appropriate body segments. The subject then performed 22 postures without the aid of the extra static body and these were used as the testing set. The subject was then instructed to perform 71 postures with the aid of the static body and these were used as the training set to identify the SESC parameters for the articulated chain. The total of 93 postures were recorded in approximately two hours.

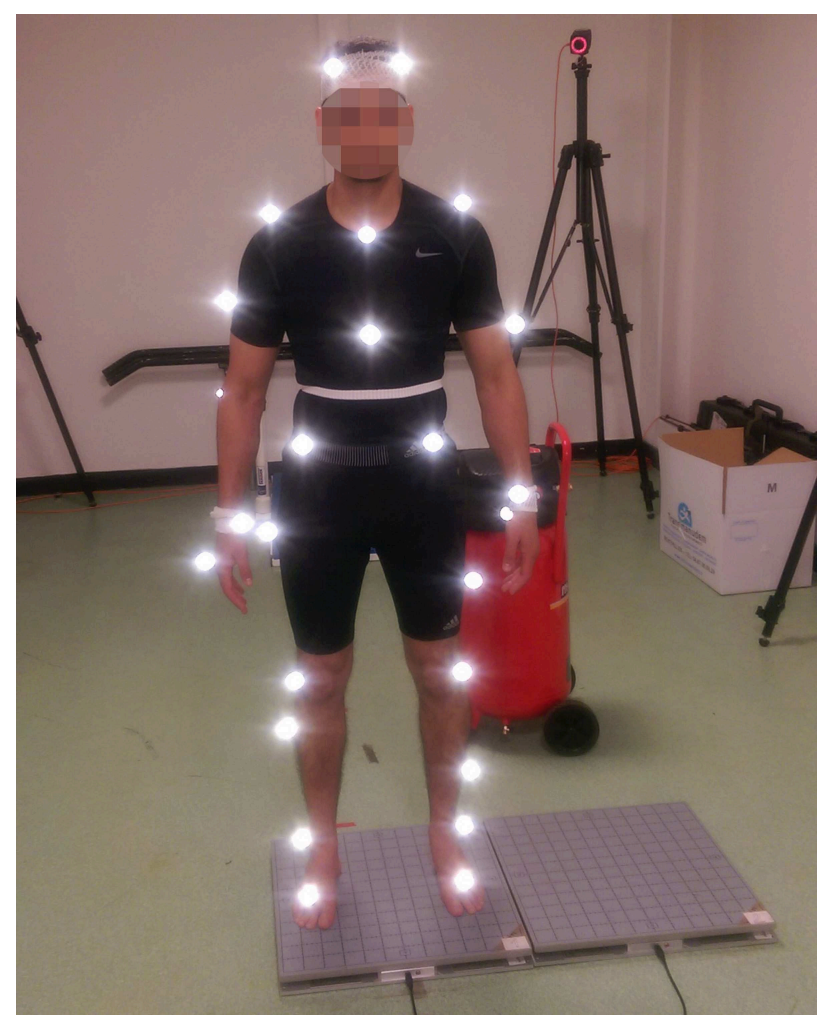

Figure 4.13: The VICON/AMTI-OR6 setup during the experimental phase shows the static body (red object not currently on the force plates used to assist in producing various number of postures while being fixed in one location on top of the force plate surface) and placement of the 35 passive markers following the Plug-in-Gait (PiG) template to define the body segments. 
The differences between the predicted $\mathrm{CoM}$ and the measured $\mathrm{CoM}$ via the force plate are shown in Tab. 4.12. The CoM in the plane of the force plate is shown to have an average distance of about $5.11 \mathrm{~mm}$ and an RMSE of $5.22 \mathrm{~mm}$ in the $x$ direction and $2.46 \mathrm{~mm}$ in the $y$ direction. The worst $\Delta x$ is $10.96 \mathrm{~mm}$ and the worst $\Delta y$ is $-5.15 \mathrm{~mm}$.

The effect of number of postures of Subject 6 to the accuracy were observed by using the 71 postures as the training set to evaluate the SESC parameters. The remaining ten postures were then used as the testing set to estimate the CoM. The average distance is plotted against the number of postures and the results are shown in Fig. 4.14. Good improvement is seen as the number of postures increased through posture 55, afterwhich the average distance remains relatively constant.

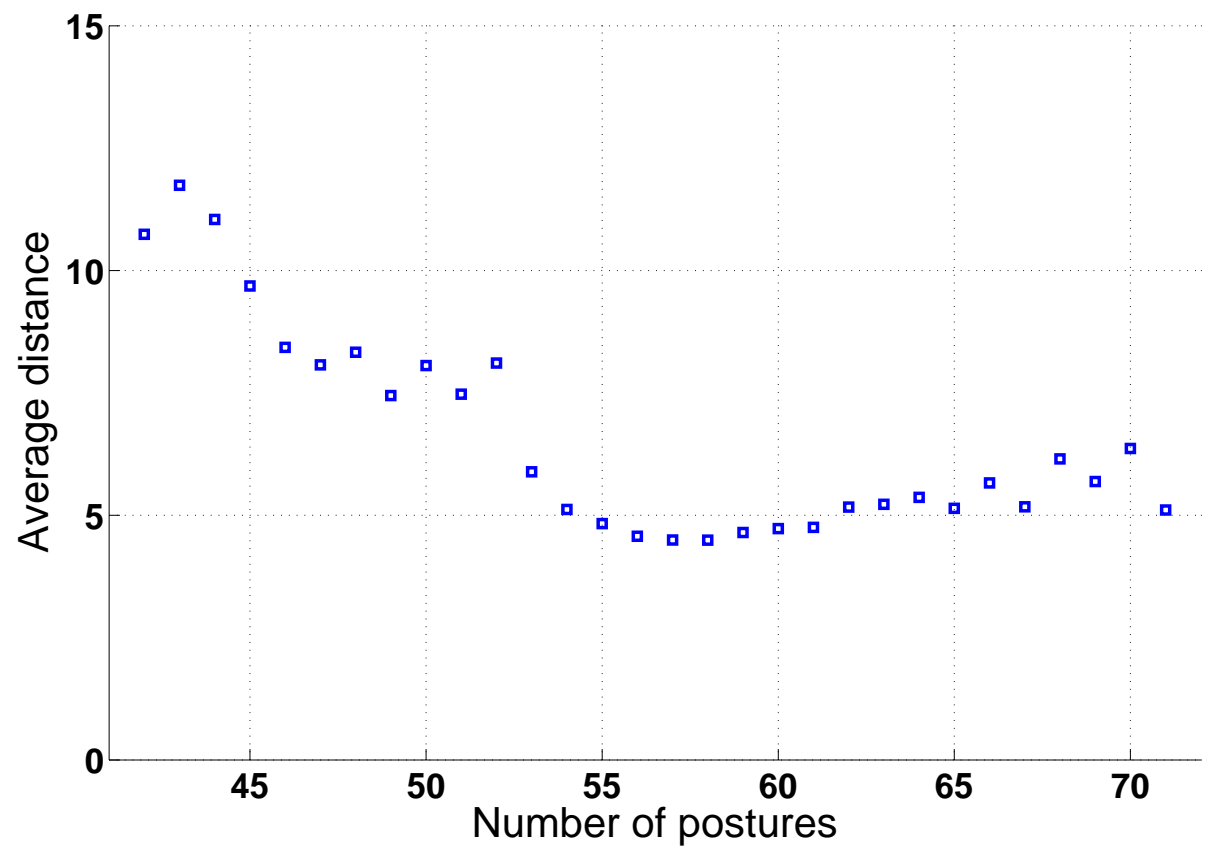

Figure 4.14: The average distance of the CoM for Subject 6 for the testing set of twenty two postures as a function of the number of experimental postures. 
Table 4.12: The CoM positions for Subject 6 as predicted by the node-based SESC method for the twenty two postures using the data from Vicon/AMTI-OR6 sensors. The table includes the deviations of the predicted CoM from the measured CoM.

\begin{tabular}{|c|c|c|c|c|c|c|c|}
\hline \multirow[b]{2}{*}{ Postures } & \multicolumn{2}{|c|}{ Predicted CoM (mm) } & \multicolumn{2}{|c|}{ Measured CoM (mm) } & \multicolumn{2}{|c|}{ Error $(\mathrm{mm})$} & \multirow{2}{*}{$\frac{\text { Distance }(\mathrm{mm})}{d}$} \\
\hline & $x$ & $y$ & $x$ & $y$ & $\Delta x$ & $\Delta y$ & \\
\hline 1 & 1.36 & 21.27 & 0.27 & 21.93 & 1.09 & -0.66 & 1.27 \\
\hline 2 & -21.91 & 274.30 & -28.40 & 274.70 & 6.50 & -0.39 & 6.51 \\
\hline 3 & -23.32 & 269.30 & -27.14 & 269.90 & 3.82 & -0.59 & 3.87 \\
\hline 4 & -22.41 & 272.50 & -23.49 & 271.60 & 1.08 & 0.89 & 1.40 \\
\hline 5 & -21.33 & 274.10 & -21.44 & 274.00 & 0.11 & 0.09 & 0.14 \\
\hline 6 & -18.52 & 275.00 & -24.61 & 280.10 & 6.09 & -5.15 & 7.98 \\
\hline 7 & -20.59 & 274.10 & -26.15 & 279.00 & 5.56 & -4.86 & 7.38 \\
\hline 8 & -25.75 & 273.30 & -30.31 & 277.10 & 4.56 & -3.83 & 5.96 \\
\hline 9 & -28.64 & 271.20 & -32.26 & 272.10 & 3.62 & -0.93 & 3.73 \\
\hline 10 & -44.38 & 372.30 & -36.26 & 368.20 & -8.12 & 4.04 & 9.07 \\
\hline 11 & 24.86 & 68.01 & 26.17 & 70.20 & -1.31 & -2.20 & 2.56 \\
\hline 12 & -18.34 & 173.00 & -13.59 & 169.70 & -4.75 & 3.26 & 5.76 \\
\hline 13 & -9.29 & 222.40 & -5.34 & 224.30 & -3.95 & -1.95 & 4.40 \\
\hline 14 & -38.51 & 191.60 & -42.33 & 188.30 & 3.82 & 3.37 & 5.09 \\
\hline 15 & -72.08 & 116.50 & -68.74 & 115.20 & -3.34 & 1.32 & 3.59 \\
\hline 16 & -17.99 & 211.60 & -14.38 & 211.90 & -3.61 & -0.27 & 3.62 \\
\hline 17 & 41.05 & 258.00 & 33.70 & 259.30 & 7.34 & -1.34 & 7.46 \\
\hline 18 & -21.67 & 181.80 & -13.96 & 181.80 & -7.71 & 0.06 & 7.71 \\
\hline 19 & 15.52 & 171.00 & 9.16 & 173.90 & 6.36 & -2.88 & 6.98 \\
\hline 20 & -16.86 & 293.10 & -14.57 & 291.90 & -2.29 & 1.13 & 2.55 \\
\hline 21 & -5.70 & 238.00 & -1.76 & 236.40 & -3.94 & 1.54 & 4.23 \\
\hline 22 & 6.42 & 288.00 & -4.54 & 286.20 & 10.96 & 1.79 & 11.10 \\
\hline Average $^{\S}$ & & & & & 4.54 & 1.93 & 5.11 \\
\hline RMSE & & & & & 5.22 & 2.46 & \\
\hline
\end{tabular}

${ }^{\S}$ For $\Delta x$ and $\Delta y$ the average is of the absolute values. 


\subsection{Accuracy of the Vertical Component of the CoM}

Force plates are not capable of measuring the vertical component of the CoM. The WBB and the AMTI-OR6 are only capable of providing the horizontal coordinates of the CoM under static conditions. Therefore, they do not validate the vertical component of the CoM.

A validation could be performed by having the subjects lay on the force plate. Laying on the force plate places the vectors in the SESC horizontally relative to ground. Thus, the lengths of the vectors need to be accurate when vertically aligned to produce an accurate CoM estimation when laying down. However, performing this type of postures is not possible due to the limitations of the MoCap systems (the Kinect and the VICON). When recording these postures the body limbs get close to each other making it problematic for the MoCap to recognize and capture the joint positions. The Kinect will become unable to track the nodes and fail to construct the motion. Similarly the VICON will lose the detection of the markers as they get obstructed by other limbs.

The validation of the vertical component can be partially performed by examining postures with more horizontal components as depicted in Fig. 4.19. The subjects are instructed to perform horizontal postures and avoid the obstruction between body limbs.

In Experiment A involving Subject 1, two such postures (56 and 85) were obtained. The CoM in the plane of the force plate is shown to have an error of $7.03 \mathrm{~mm}$ in the $x$ direction and $10.27 \mathrm{~mm}$ in the $y$ direction for posture 56 . The error for posture 85 is $-5.97 \mathrm{~mm}$ in the $x$ direction and -7.5 $\mathrm{mm}$ in the $y$ direction as depicted in Tab. 4.1.

In Experiment B with Subject 2, two horizontal-like postures (86 and 92) were obtained. The $\mathrm{CoM}$ in the plane of the force plate is shown to have an error of $-5.65 \mathrm{~mm}$ in the $x$ direction and $9.64 \mathrm{~mm}$ in the $y$ direction for posture 86 . The error for posture 92 is $10.49 \mathrm{~mm}$ in the $x$ direction and $-9.65 \mathrm{~mm}$ in the $y$ direction as depicted in Tab. 4.4. 


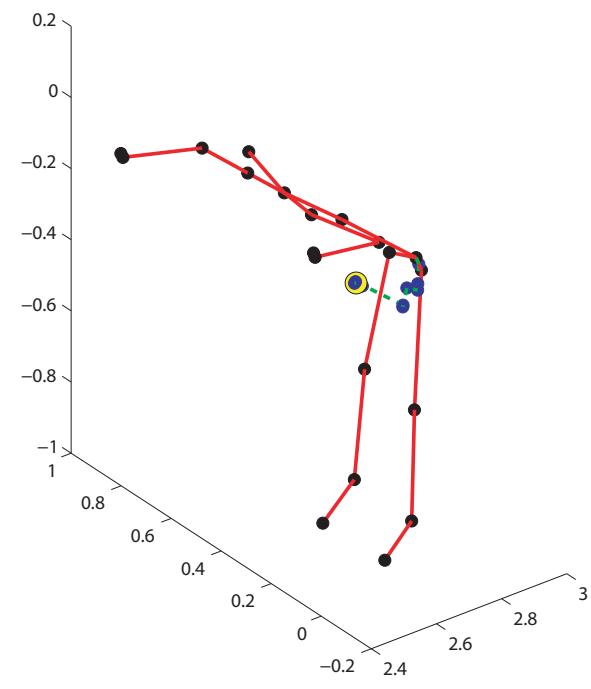

(a)

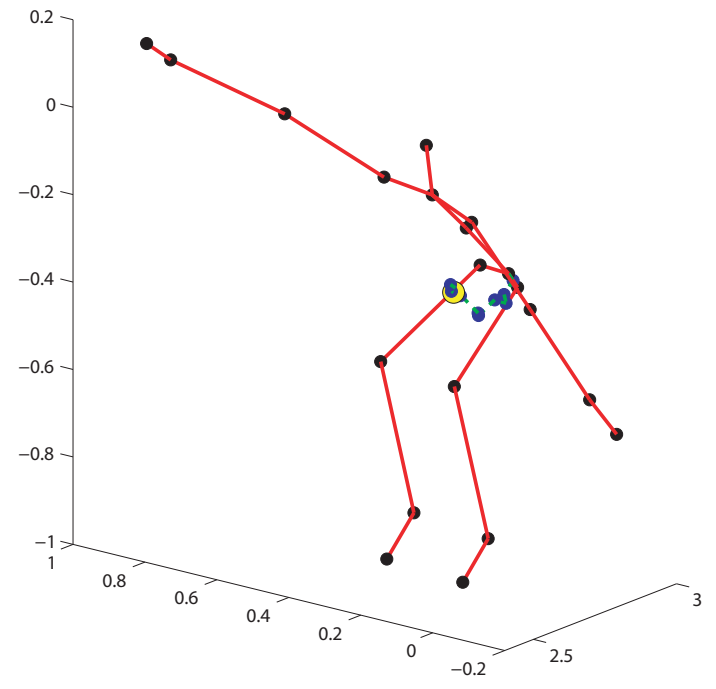

(b)

Figure 4.15: The two postures with more horizontal components used to validate the vertical component of the CoM of Subject 1, (a) posture number 56 and (b) posture number 85 , as depicted in Tab. 4.1.

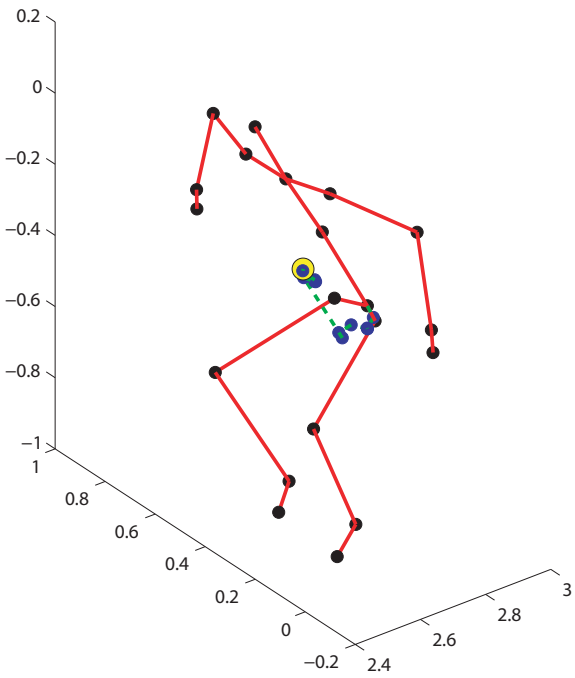

(a)

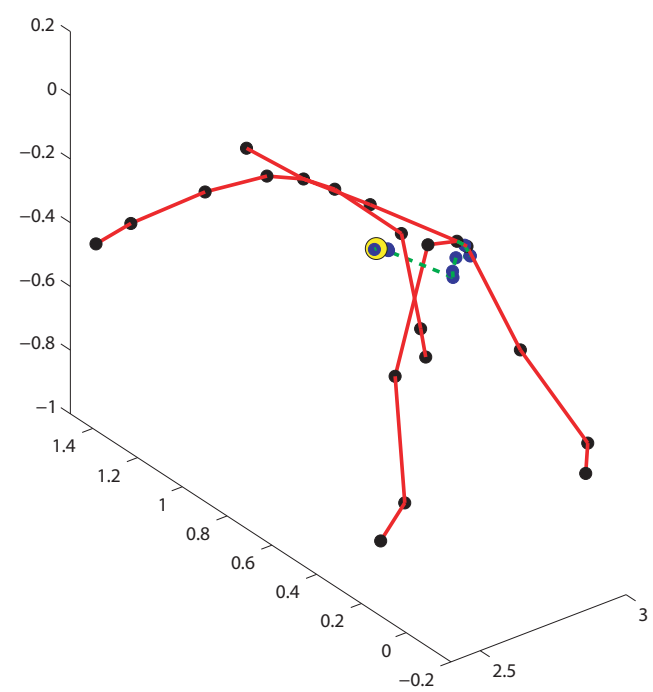

(b)

Figure 4.16: The two postures with more horizontal components used to validate the vertical component of the CoM of Subject 2, (a) posture number 86 and (b) posture number 92, as depicted in Tab. 4.4. 
In Experiment $\mathrm{C}$ performed by Subject 3, two horizontal-like postures ( 7 and 31) were obtained. The CoM in the plane of the force plate is shown to have an error of $4.5 \mathrm{~mm}$ in the $x$ direction and $10.84 \mathrm{~mm}$ in the $y$ direction for posture 7 . The error for posture 31 is $-5.86 \mathrm{~mm}$ in the $x$ direction and $5.34 \mathrm{~mm}$ in the $y$ direction as depicted in Tab. 4.5.

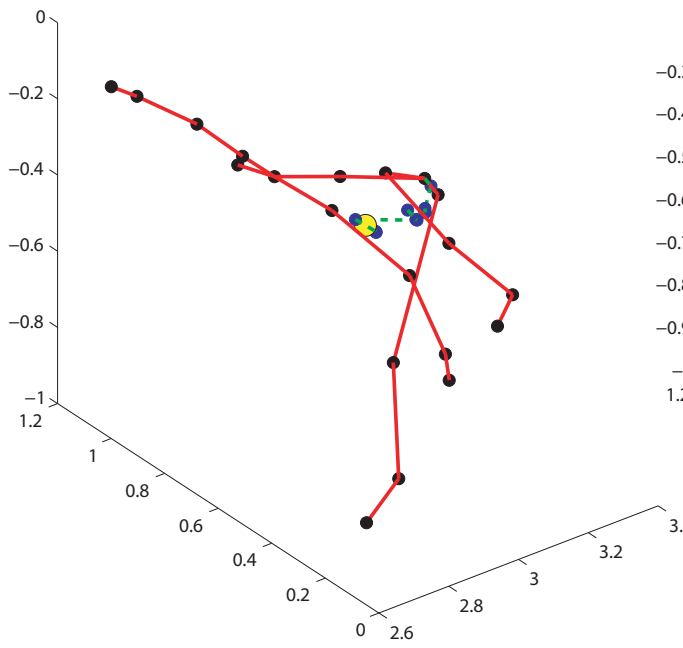

(a)

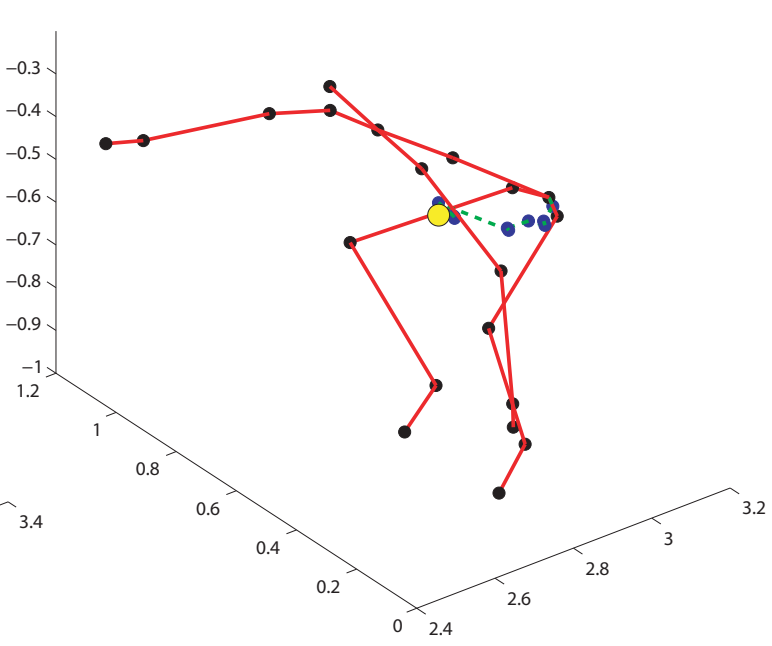

(b)

Figure 4.17: The two postures with more horizontal components used to validate the vertical component of the CoM of Subject 3, (a) posture number 7 and (b) posture number 31, as depicted in Tab. 4.5.

In Experiment D carried out by Subject 4, two horizontal-like postures (12 and 27) were obtained. The CoM in the plane of the force plate is shown to have an error of $-9.06 \mathrm{~mm}$ in the $x$ direction and $-10.59 \mathrm{~mm}$ in the $y$ direction for posture 12 . The error for posture 27 is $5.04 \mathrm{~mm}$ in the $x$ direction and $10.03 \mathrm{~mm}$ in the $y$ direction as depicted in Tab. 4.6.

In Experiment F performed by Subject 6 utilizing VICON/AMTI-OR6 sensors, two horizontallike postures (17 and 18) were obtained. The CoM in the plane of the force plate has an error of 7.3 $\mathrm{mm}$ in the $x$ direction and $-1.3 \mathrm{~mm}$ in the $y$ direction for posture 17 . The error for posture 18 is 


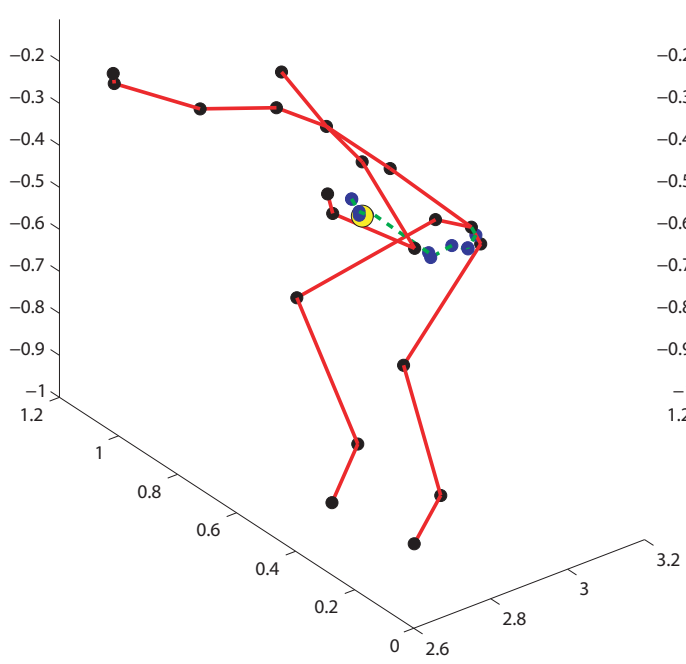

(a)

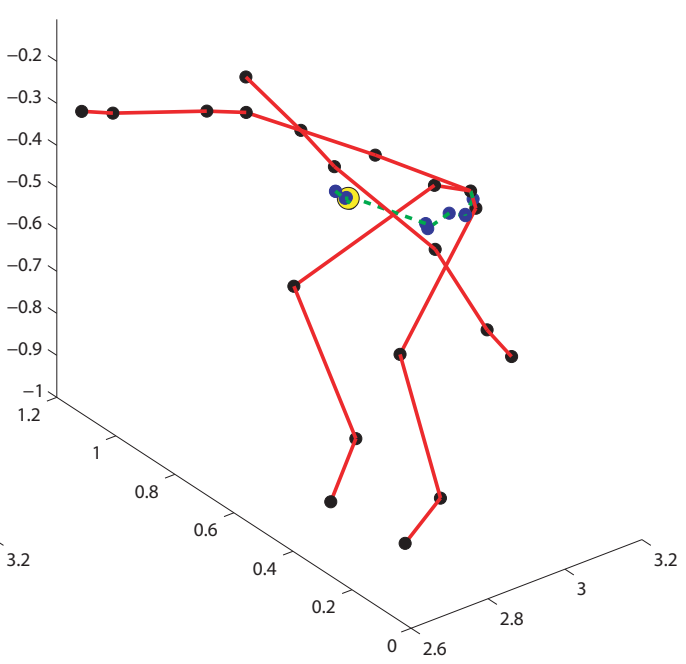

(b)

Figure 4.18: The two postures with more horizontal components used to validate the vertical component of the CoM of Subject 4, (a) posture number 12 and (b) posture number 27, as depicted in Tab. 4.6.

$-7.7 \mathrm{~mm}$ in the $x$ direction and less than $0.1 \mathrm{~mm}$ in the $y$ direction as depicted in Tab. 4.12. Note that the error is similar to other experiments.

For the experiments involving the validation of the vertical component, the worst error in $x$ and $y$ were $10.94 \mathrm{~mm}$ and $10.84 \mathrm{~mm}$, respectively. In contrast, the minimum error in $x$ and $y$ were 4.5 $\mathrm{mm}$ and less than $0.1 \mathrm{~mm}$, respectively. 


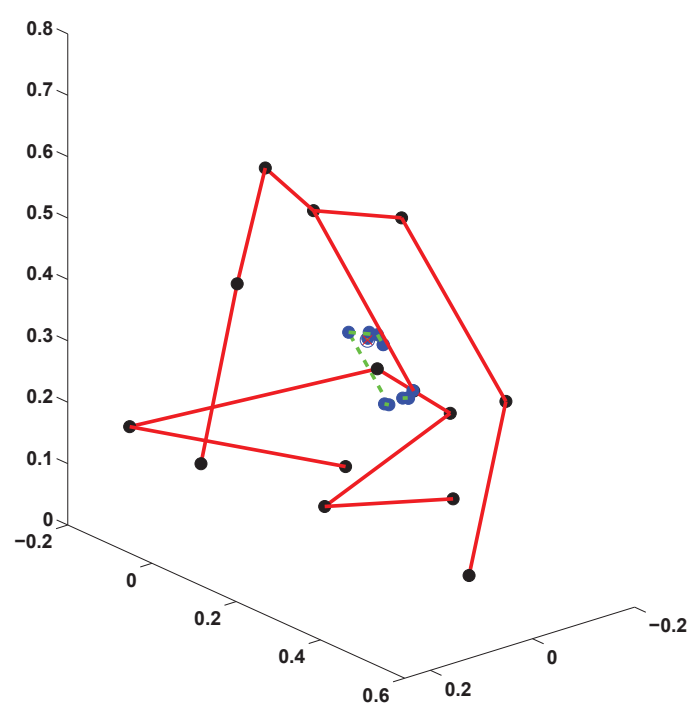

(a)

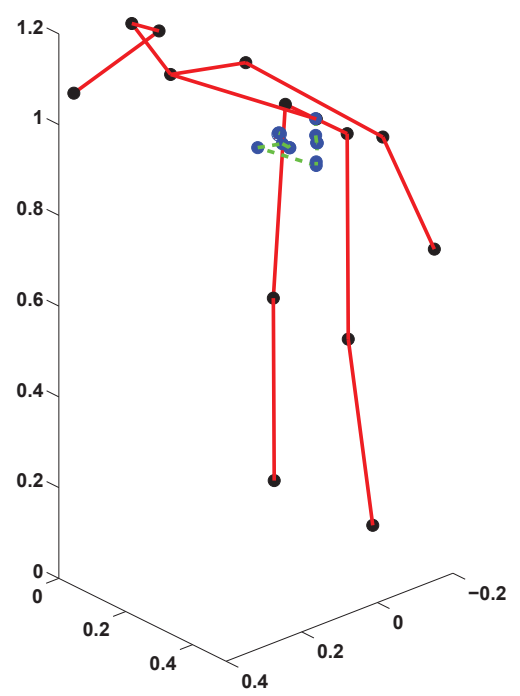

(b)

Figure 4.19: The two postures with horizontal-like components used to validate the vertical component of the CoM of Subject 6 in experiment F, (a) posture number 17 and (b) posture number 18 , as depicted in Tab. 4.12. 


\section{CHAPTER V}

\section{CONCLUSIONS AND FUTURE WORK}

\subsection{Contributions of the Dissertation}

The presented work introduced seven main contributions to the literature. The first one is the

development of the node-based SESC modeling technique for estimating the CoM of humanoid robots and human beings. This technique is a modification to the original Statically Equivalent Serial Chain modeling by relying on joint positions (nodes) rather than joint angles. Accordingly, this eliminates the need for calculating the joint angles by utilizing any MoCap system position data directly and reduces the processing time.

The second contribution is the development of the method for finding the relative orientation (A) and displacement $(\vec{d})$ between the Kinect and the WBB.

The third contribution is the development of the node-based SESC donor model technique to estimate the SESC parameters based on someone else's SESC model. The donor model can estimate the SESC parameters of subjects with limited body motion using fewer postures. The donor model is capable of generating an accurate estimate of the subject's CoM.

The fourth contribution is an extension to the node-based SESC modeling to include an extra static body to the modeling process. The introduction of an extra static body such as wheelchairs 
or walkers will improve the stability of elderly people or when preforming unconventional body postures. As a result, this will impact and minimize the time required for capturing static postures.

The fifth contribution is the further extension to the node-based SESC modeling allowing an arbitrary location of the CoM by introducing a relevant moving frame. This process will account for bodies whose CoM does not lie along the vector between nodes.

The sixth contribution is the development of the node-based SESC modeling technique for estimating the CoM without the advance knowledge of $\mathbf{A}$ and $\vec{d}$ between the MoCap system and the force plate reference frames. Although this modeling requires a relatively large number of postures, the process does not require any previous calibration.

The final contribution is the processing algorithm that was built using the Windows Presentation Foundation system. This algorithm is capable of processing and recording the data coming from the Kinect and the WBB simultaneously. The algorithm is capable of sensing the user's location. The data capturing is triggered when the user is standing on the WBB and stopped automatically when the user is no longer standing on the WBB. The algorithm is capable of determining static postures and will alert the user once a posture is deemed static.

The presented contributions of this dissertation were validated on several human subjects via the use of Kinect/WBB and VICON/AMTI-OR6 sensors. The node-based SESC technique produced rapid and accurate estimation of the CoM when applied to humans.

\subsection{Future Work}

Implementing the node-based SESC method for biomechanics studies, for example, in sports and health science requires further validation. The validation can be accomplished by utilizing different force plates in a single MoCap environment. The first force plate can be used during the 
experimental construction of the SESC parameters. The subject then moves to the second force plate. The data from the second force plate can validate the model accuracy.

The experimental construction of the SESC parameters is influenced by many factors. An optimization study of these factors would be valuable. For example, the process can be optimized by more thoroughly investigating the effect of the number of postures to the model accuracy. In order to improve the identification phase, a further refinement of the data collection process is required. The experimentation will determine the possible number of frames (or the window of time) required to generate a static posture. A possible improvement that needs to be determined is the required standard deviation for the nodes and the CoP to accurately gauge if the posture is static.

The development of the node-based SESC modeling technique does not implement any type of filtering techniques. The literature includes a variety of filtering techniques, for example, the Kalman Filter, which can be utilized to determine a constant value from noisy measurements. 


\section{APPENDIX A}

\section{INSTRUCTIONAL GUIDE FOR THE EXPERIMENTAL SETUP AND POSCAP, THE POSTURE CAPTURE SOFTWARE}

\section{A.1 Introduction}

This chapter guides a user through the experimental setup and use of the tools described in this dissertation. This appendix is specific to the Kinect and the WBB sensors. Section A.2 presents the required software and hardware necessary to operate the Posture Capture Software (PosCap). PosCap is the name given to the software tool developed to implement the concepts in this dissertation. The necessary steps to connect the WBB to a Bluetooth enabled computer is described in Section A.3. Section A.4 details the instructions for the placement of the Kinect and WBB. Section A.5 presents the steps for using PosCap to capture the needed data to establish the relative location of the Kinect and WBB. The necessary information for setting up the experiment and executing PosCap to capture the near static postures is detailed in the final section. A PC running Windows 8.1 was used to develop and execute the experiments for the work covered in this dissertation.

\section{A.2 Software Download}

The PosCap software was developed using Visual Studio and written in the C-sharp (C\#) language. The user must: 
- Download and install the Kinect SDK by clicking on or scanning the code shown in Fig. A.1. This will enable PosCap and the computer to recognize the Kinect. Note that the recommended hardware configuration includes 64-bit (x64) processor, 4 GB Memory (or more), physical dualcore $3.1 \mathrm{GHz}$ (2 logical cores per physical) or faster processor, USB 3.0 controller dedicated to the Kinect for Windows v2 sensor, and DX11 capable graphics adapter.

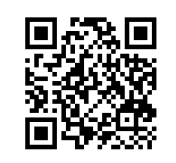

Figure A.1: Click on the code or scan to open the link for downloading the Kinect SDK.

- Download and install either Visual Studio Express or Visual Studio Community by clicking on or scanning the code shown in Fig. A.2. Visual Studio will allow the user to run the PosCap software and allow for edits, if necessary.

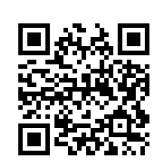

Figure A.2: Click on the code or scan to open the link for downloading the Visual Studio software.

\section{A.3 Connecting the WBB to the PC}

To connect the WBB to the PC, the user needs to do the following:

- Press and release the 'Sync' button shown in Fig. A.3 to make the WBB discoverbale. 
- On the PC go to Start $\Rightarrow$ select the device $\Rightarrow$ Pair (if the pairing was successful the LED should be blinking). Note that the WBB should be available under the list of devices as 'Nintendo RVL-WBC-01'.

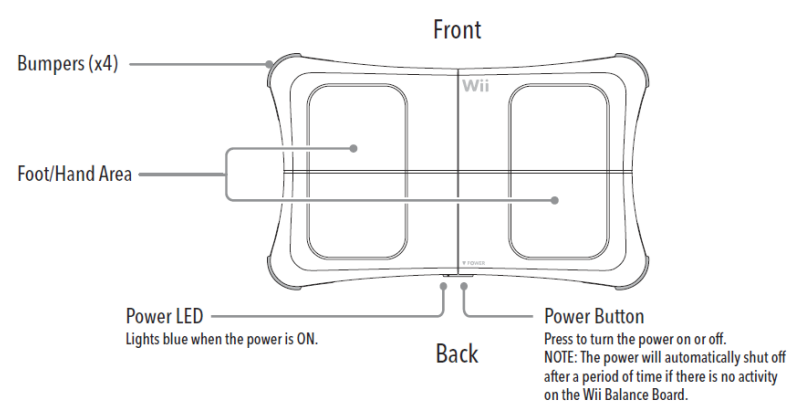

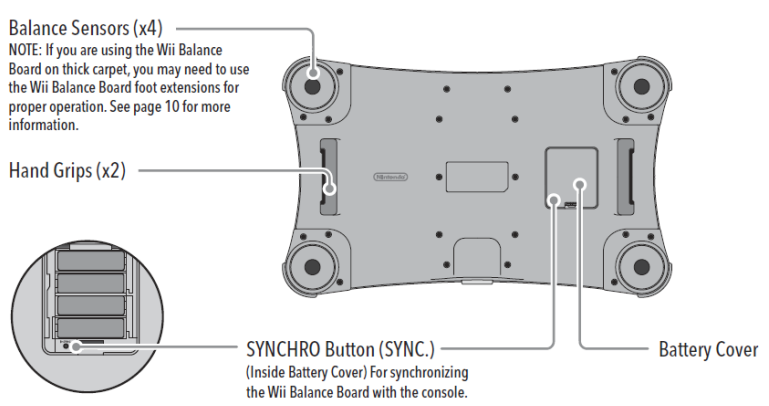

(a)

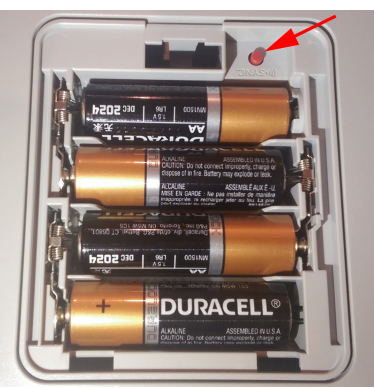

(b)

Figure A.3: The schematic of the WBB sensor, (a) the synchronizing button used to connect the device to the PC is located at the middle right, (b) the sync button is located adjacent to the battery compartment.

\section{A.4 Kinect and WBB Placement}

Concerns when placing sensors and for the experiment:

- The Kinect must be placed near the edge of a flat and stable surface. 
- The Kinect must be positioned between 0.6 meters and 1.8 meters from the floor as depicted in Fig. A.4. Note that in the experiments covered in this dissertation the sensor was placed approximately 0.8 meters above the floor.

- Avoid positioning the Kinect in direct sunlight.

- The Kinect has a depth range of 0.5 meters to 4.5 meters as shown in Fig. A.4. To facilitate full-body tracking, the WBB was positioned between 2.3 and 2.7 meters.

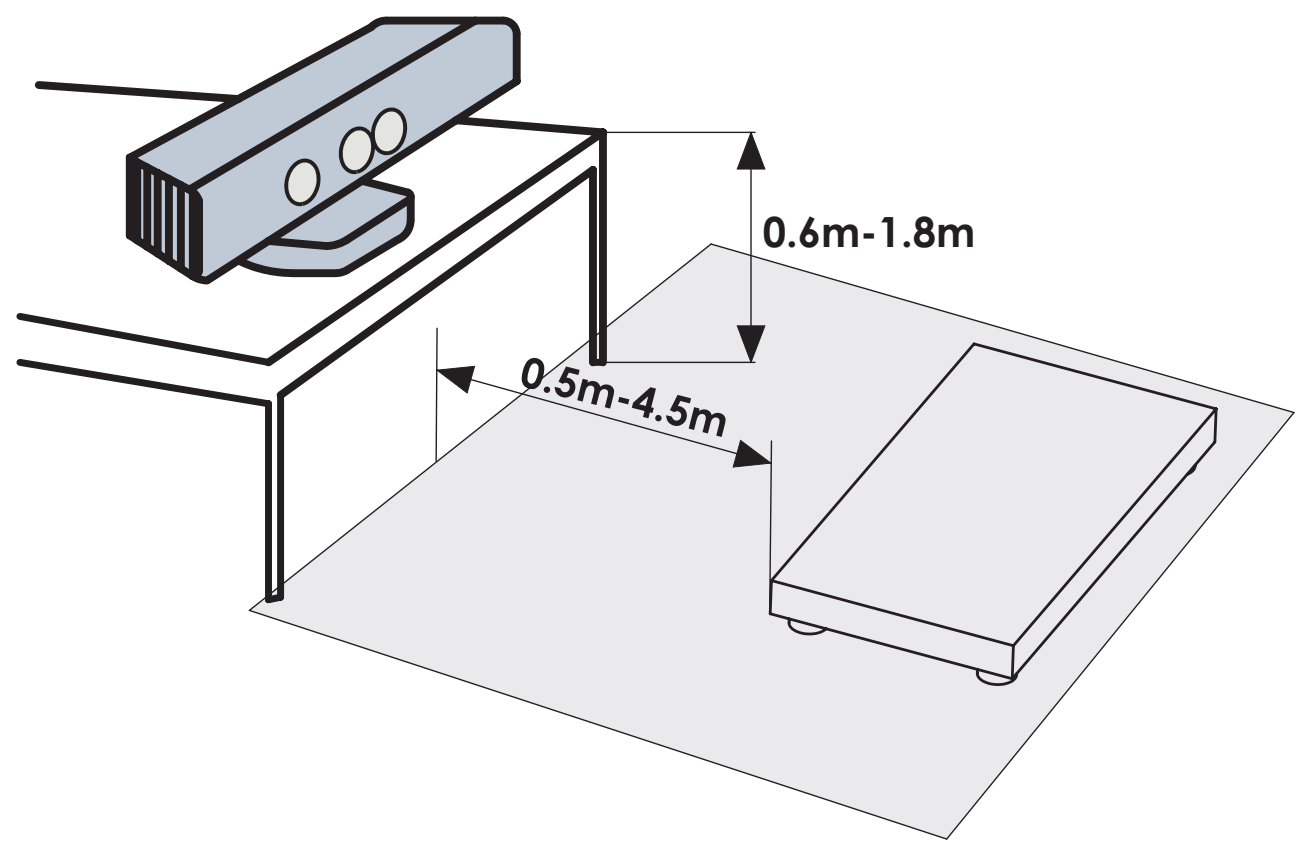

Figure A.4: The placement of Kinect and WBB sensors.

\section{A.5 Parameter Identification using PosCap}

The relative position between the WBB and the Kinect can be found using the method in Chapter II. Given that the steps and comments in A.3 and A.4 were used: 
- Run the PosCap software and check the box next to 'WBB' as shown in Fig. A.5 (a sound notification will be triggered if the connection was successful and the LED state will change to solid).

- To choose where to save the generated data file, click on the circle next to 'Save File' located on the same line containing the WBB check box as shown in Fig. A.5.

- Start the experiment by placing the weight on the WBB as shown in Fig. A.5. The target should face the Kinect sensor.

- Locate the weight on the PosCap screen and click on the target to record the position/CoP data (a sound notification occurs indicating the click action). Avoid positioning the target at an angle as the Kinect works best when facing a flat frontal surface. Continue moving the target weight to different positions and recording the position/CoP data until obtaining the required readings.

\section{A.6 Capturing Postures using the PosCap}

Given the force plate location has been performed according to the instructions in A.5:

- Uncheck the box next to 'WBB', then check the box next to 'Body Tracking' shown in Fig. A.5 (a sound notification occurs if the connection is successful and the WBB LED state will change to solid).

- To choose where to save the generated data file, click on the circle next to 'Save File' located on the same line containing the 'Body Tracking' check box as illustrated in Fig. A.5.

- Start the experiment by standing on the WBB and performing the required postures. The software will detect the user once on the WBB and initiate the data capturing.

- Note that the Kinect sensor performs well when faced frontally and the accuracy decreases as the body turns away from the sensor. 


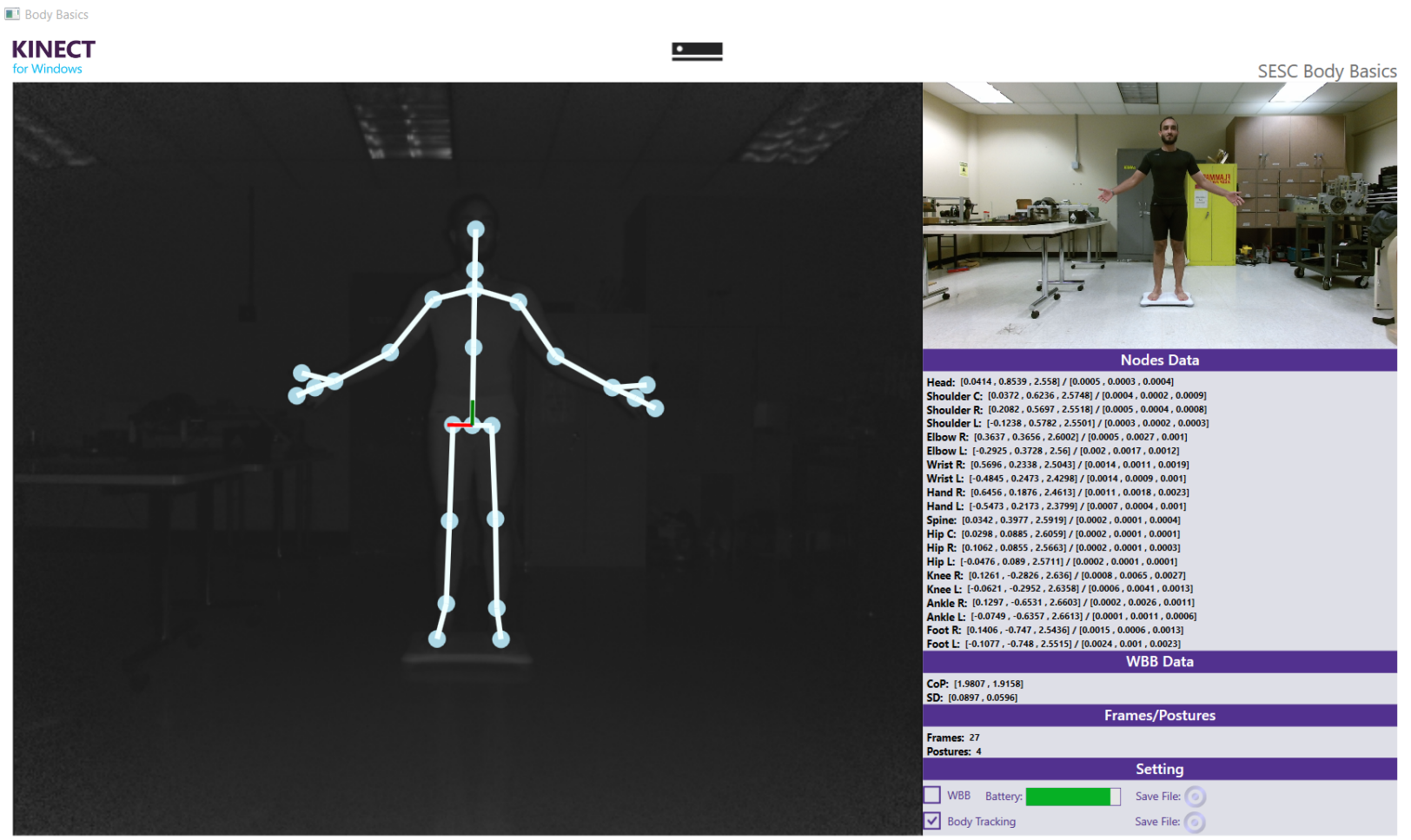

Figure A.5: The Posture Capture software (PosCap), best used when the subject is wearing a tight, non-reflective outfit and required to be standing on the WBB. 
- Avoid having more than one subject in the workspace of the Kinect sensor during the experiment. The PosCap software is only capable of handling one subject during the test.

- Avoid having chairs or walkers in the workspace because the Kinect sensor might detect these objects as human subjects. 


\section{BIBLIOGRAPHY}

[1] http://www.xbox.com/en-US/kinect.

[2] http://www.nintendo.com/consumer/downloads/wiiBalanceBoard.pdf.

[3] http://www.bleng.com/reflective-markers/25.4mm.

[4] http://www.ndigital.com.

[5] http://www.kistler.com/us/en/index.

[6] http://www.amti.biz/.

[7] L. Sentis, "Synthesis and control of whole-body behaviors in humanoid systems," Ph.D. dissertation, Citeseer, 2007.

[8] M. G. Gaerlan, "The role of visual, vestibular, and somatosensory systems in postural balance," Ph.D. dissertation, UNIVERSITY OF NEVADA, LAS VEGAS, 2010.

[9] D. A. Winter, "Human balance and posture control during standing and walking," Gait \& posture, vol. 3, no. 4, pp. 193-214, 1995.

[10] J. J. Buchanan and F. B. Horak, "Emergence of postural patterns as a function of vision and translation frequency," Journal of Neurophysiology, vol. 81, no. 5, pp. 2325-2339, 1999.

[11] K. F. Zabjek, C. Coillard, C.-H. Rivard, and F. Prince, "Estimation of the centre of mass for the study of postural control in idiopathic scoliosis patients: a comparison of two techniques," European Spine Journal, vol. 17, no. 3, pp. 355-360, 2008.

[12] S. E. Halliday, D. A. Winter, J. S. Frank, A. E. Patla, and F. Prince, "The initiation of gait in young, elderly, and parkinson's disease subjects," Gait \& posture, vol. 8, no. 1, pp. 8-14, 1998.

[13] M. Agmon, C. K. Perry, E. Phelan, G. Demiris, and H. Q. Nguyen, "A pilot study of wii fit exergames to improve balance in older adults," Journal of Geriatric Physical Therapy, vol. 34, no. 4, pp. 161-167, 2011. 
[14] A. Baca and P. Kornfeind, "Rapid feedback systems for elite sports training," Pervasive Computing, IEEE, vol. 5, no. 4, pp. 70-76, 2006.

[15] M. A. Jaffrey, "Estimating centre of mass trajectory and subject-specific body segment parameters using optimisation approaches," Ph.D. dissertation, Victoria University, 2008.

[16] H. M. Schepers, E. H. van Asseldonk, J. H. Buurke, and P. H. Veltink, "Ambulatory estimation of center of mass displacement during walking," Biomedical Engineering, IEEE Transactions on, vol. 56, no. 4, pp. 1189-1195, 2009.

[17] P. De Leva, "Adjustments to zatsiorsky-seluyanov's segment inertia parameters," Journal of biomechanics, vol. 29, no. 9, pp. 1223-1230, 1996.

[18] D. A. Winter, Biomechanics and motor control of human movement. John Wiley \& Sons, 2009.

[19] T. Shimba, "An estimation of center of gravity from force platform data," Journal of Biomechanics, vol. 17, no. 1, pp. 53-60, 1984.

[20] D. L. King and V. M. Zatsiorsky, "Extracting gravity line displacement from stabilographic recordings," Gait \& Posture, vol. 6, no. 1, pp. 27-38, 1997.

[21] G. Venture, K. Ayusawa, and Y. Nakamura, "Real-time identification and visualization of human segment parameters," in Engineering in Medicine and Biology Society, 2009. EMBC 2009. Annual International Conference of the IEEE. IEEE, 2009, pp. 3983-3986.

[22] A. Betker, Z. Moussavi, and T. Szturm, "Center of mass function approximation," in Engineering in Medicine and Biology Society, 2004. IEMBS'04. 26th Annual International Conference of the IEEE, vol. 1. IEEE, 2004, pp. 687-690.

[23] A. Betker, T. Szturm, and Z. Moussavi, "Application of feedforward backpropagation neural network to center of mass estimation for use in a clinical environment," in Engineering in Medicine and Biology Society, 2003. Proceedings of the 25th Annual International Conference of the IEEE, vol. 3. IEEE, 2003, pp. 2714-2717.

[24] S. L. Wolf, H. X. Barnhart, N. G. Kutner, E. McNeely, C. Coogler, and T. Xu, "Selected as the best paper in the 1990s: Reducing frailty and falls in older persons: An investigation of tai chi and computerized balance training," Journal of the American Geriatrics Society, vol. 51, no. 12, pp. 1794-1803, 2003.

[25] F. Massaad, T. M. Lejeune, and C. Detrembleur, "Reducing the energy cost of hemiparetic gait using center of mass feedback: a pilot study," Neurorehabilitation and neural repair, 2009.

[26] S. Cotton, A. Murray, and P. Fraisse, "Estimation of the center of mass using statically equivalent serial chain modeling," in ASME 2009 International Design Engineering Technical Conferences and Computers and Information in Engineering Conference. American Society of Mechanical Engineers, 2009, pp. 961-967. 
[27] _ - "Statically equivalent serial chains for modeling the center of mass of humanoid robots," in Humanoid Robots, 2008. Humanoids 2008. 8th IEEE-RAS International Conference on. IEEE, 2008, pp. 138-144.

[28] S. Cotton, A. P. Murray, and P. Fraisse, "Estimation of the center of mass: from humanoid robots to human beings," Mechatronics, IEEE/ASME Transactions on, vol. 14, no. 6, pp. 707$712,2009$.

[29] B. Espiau, R. Boulic et al., "On the computation and control of the mass center of articulated chains," 1998.

[30] O. Fischer, "Uber die reduzierten systeme und die hauptpunkte der glieder eines gelenkmechanismus und ihre bedeutung for die technische mechanik," Z. f. math. Phys, vol. 47, pp. 429466, 1902.

[31] I. I. Artobolevskii, Theory of Mechanisms and Machines (Russian), 2nd ed. Gos. Izdat. Tekh. Teoret. Lit., Moscow, 1951.

[32] B. Dizloglo, "Band 3, dynamik," in Getriebelehre. Vieweg, Braunschweig, 1966, p. 199.

[33] V. P. Goryachkin, "The forces of inertia and their balancing," in Collected Works (Russian). lzd, Kolos, Moscow, (1914); (1965), vol. I, pp. 283-418 (720).

[34] N. I. Manolescu and D. Maros, "Teoria mecanismelor şi a maşinilor-cinematica roţilor dinţate," EdituraTehnică, Bucureşti, 1958.

[35] V. A. Yudin, "The balancing of machines and their stability (Russian)." lzd. Voenno-Inzh. Akad. Krasnoi Armii, Moscow, 1941, p. 124.

[36] R. Berkof and G. Lowen, "A new method for completely force balancing simple linkages," Journal of Manufacturing Science and Engineering, vol. 91, no. 1, pp. 21-26, 1969.

[37] A. González, M. Hayashibe, and P. Fraisse, "Estimation of the center of mass with kinect and wii balance board," in Intelligent Robots and Systems (IROS), 2012 IEEE/RSJ International Conference on. IEEE, 2012, pp. 1023-1028.

[38] — - "Three dimensional visualization of the statically equivalent serial chain from kinect recording," in Engineering in Medicine and Biology Society (EMBC), 2012 Annual International Conference of the IEEE. IEEE, 2012, pp. 4843-4846.

[39] — - "Online identification and visualization of the statically equivalent serial chain via constrained kalman filter," in Robotics and Automation (ICRA), 2013 IEEE International Conference on. IEEE, 2013, pp. 5323-5328.

[40] J. Richards, "Biomechanics in clinic and research," Philadelphia: Churchill Livingstone, 2008.

[41] E. Muybridge, Animal locomotion. Da Capo Press New York, 1969. 
[42] E. Muybridge, E. Muybridge, E. Muybridge, and E. Muybridge, The human figure in motion: an electro-photographic investigation of consecutive phases of muscular actions. Chapman and Hall, 1901.

[43] E.-J. Marey, "Le fusil photographique," Bulletin de la Société française de photographie, vol. 28, pp. 127-33, 1882.

[44] H. J. Woltring, "Planar control in multi-camera calibration for 3-d gait studies," Journal of Biomechanics, vol. 13, no. 1, pp. 39-48, 1980.

[45] http://www.vicon.com.

[46] http://www.motionanalysis.com.

[47] http://www.qualisys.com.

[48] http://www.organicmotion.com/company/.

[49] Z. Zhang, "Microsoft kinect sensor and its effect," MultiMedia, IEEE, vol. 19, no. 2, pp. 4-10, 2012.

[50] E. E. Stone and M. Skubic, "Evaluation of an inexpensive depth camera for passive in-home fall risk assessment," in Pervasive Computing Technologies for Healthcare (PervasiveHealth), 2011 5th International Conference on. IEEE, 2011, pp. 71-77.

[51] R. A. Clark, Y.-H. Pua, K. Fortin, C. Ritchie, K. E. Webster, L. Denehy, and A. L. Bryant, "Validity of the microsoft kinect for assessment of postural control," Gait \& posture, vol. 36, no. 3, pp. 372-377, 2012.

[52] S. Obdrzalek, G. Kurillo, F. Ofli, R. Bajcsy, E. Seto, H. Jimison, and M. Pavel, "Accuracy and robustness of kinect pose estimation in the context of coaching of elderly population," in Engineering in Medicine and Biology Society (EMBC), 2012 Annual International Conference of the IEEE. IEEE, 2012, pp. 1188-1193.

[53] http://www.visualstudio.com/.

[54] http://msdn.microsoft.com/en-us/library/kx37x362.aspx.

[55] J. Deutsch, D. Robbins, J. Morrison, and P. G. Bowlby, "Wii-based compared to standard of care balance and mobility rehabilitation for two individuals post-stroke," in Virtual Rehabilitation International Conference, 2009. IEEE, 2009, pp. 117-120.

[56] M. W. Kennedy, J. P. Schmiedeler, C. R. Crowell, M. Villano, A. D. Striegel, and J. Kuitse, "Enhanced feedback in balance rehabilitation using the nintendo wii balance board," in $e$ Health Networking Applications and Services (Healthcom), 2011 13th IEEE International Conference on. IEEE, 2011, pp. 162-168. 
[57] R. A. Clark, A. L. Bryant, Y. Pua, P. McCrory, K. Bennell, and M. Hunt, "Validity and reliability of the nintendo wii balance board for assessment of standing balance," Gait \& posture, vol. 31, no. 3, pp. 307-310, 2010.

[58] B. Peek, "Brianpeek. com," WiimoteLib-.NET Managed Library for the Nintendo Wii Remote, URL (luglio 2010): http://www. brianpeek. com/blog/pages/wiimotelib. aspx.

[59] D. A. Winter, A. E. Patla, F. Prince, M. Ishac, and K. Gielo-Perczak, "Stiffness control of balance in quiet standing," Journal of neurophysiology, vol. 80, no. 3, pp. 1211-1221, 1998.

[60] D. A. Winter, A. E. Patla, M. Ishac, and W. H. Gage, "Motor mechanisms of balance during quiet standing," Journal of Electromyography and Kinesiology, vol. 13, no. 1, pp. 49-56, 2003.

[61] P. M. Larochelle, A. P. Murray, and J. Angeles, "A distance metric for finite sets of rigid-body displacements via the polar decomposition," Journal of Mechanical Design, vol. 129, no. 8, pp. 883-886, 2007.

[62] R. Drillis, R. Contini, and M. Bluestein, "Body segment parameters," Artificial limbs, vol. 8, no. 1, pp. 44-66, 1964.

[63] https://msdn.microsoft.com/en-us/library/hh973073.aspx. 